\title{
European Critical Care Nursing: Working Together for a Better Tomorrow. 2011 congress
} abstracts

\section{European federation of Critical Care Nursing associations}

Key Words: abstracts * congress * critical care * Europe * nursing *

Website: www.efccna.org

ABSTRACTS: ORAL PRESENTATIONS

SESSION 1: RESUSCITATION AND CARDIOVASCULAR CARE

Cardio-pulmonary resuscitation with chest compression only: is it more effective?

\section{Marios Georgiou, Cyprus}

Email: mariogrn@spidernet.com.cy

Aim: To explore the hypothesis whether chest-compression only CPR (CC-CPR) delivered lay rescuers during the out-of-hospital cardiac arrest is superior over the conventional (e.g. chest compression plus mouth-to-mouth ventilation (CPR+MMV) resuscitation is supported by the published evidences. Methods: Literature review through search of Medline (Pubmed), Embase, Cochrane Library for the following text word phrases: "chest compression only", "continuous chest compression", "CCC-CPR", "no ventilation AND resuscitation", "resuscitation without ventilation". Search for the same text word phrases in the on-line database of "Resuscitation", "Circulation", "Lancet" Results: There are no human studies that compared compression only CPR with survival following conventional CPR with 30:2 compressions: ventilation ratio. Three studies suggest that continuous chest compressions without ventilation during basic life support (BLS) has better outcome compared to the BLS with ventilation. There are 11 studies that failed to demonstrate a difference in survival when chest compressions were compared to BLS using either a 15:2 or 5:1 compression: ventilation ratio. For a subgroup of patients resuscitated for longer than 15 minutes one study suggested that chest compression only basic life support has worse outcome than BLS with ventilation. This is also supported by five animal studies. Conclusion: There is insufficient evidence to support for or against compression-only bystander CPR for all victims of Cardiac arrest. Therefore, and according to ERC guidelines 2005, compression-only CPR may be considered particularly for witnessed adult sudden cardiac arrest in cases where the rescuer is unable or unwilling to start chest compression CPR with ventilations. References: Abe, T., Y. Tokuda, and S. Ishimatsu, Predictors for good cerebral performance among adult survivors of out-of-hospital cardiac arrest. Resuscitation, 2009. 80(4): p. 431-6. Iwami, T., T. Kawamura, et al. (2007). "Effectiveness of bystanderinitiated cardiac-only resuscitation for patients with out-of-hospital cardiac arrest." Circulation 116(25): 2900-7.
What you don't want to hear about cardio-pulmonary resuscitation?

Matteo Bartolomei, Stefano Musolesi, Massimo Izzi, Ilaria Sandretti, Alessandro Monesi, Roberto lacenda, Christian Sabbioni, Italy

Email: M.Bartolomei@118er.it

The aim of this study is to find out improving methods for CPR by the emergency system planning staff. From 2004 to 2008 in our working unit data on CPR cases were collected (timing, tracks recorded by the ADE monitors, Utstein style informations, provided by the intervened staff). Data analysis showed that there is a deviation between the requirements of BLSD guidelines and resuscitation quality performed. When we tried a comparison to see if this problem exists also outside of our unit, and if it had been faced and how. The research was conducted on Medline using the following key words: Cardiopulmonary resuscitation, outcome, skills. We considered the 2005yBLSD guidelines. So we reviewed the performance valuation methods, the impact assessement activities of the operators, the ability of operators themselves to accept the proposed changes and possible solution to improve the professional performance. When we analyzed the literature search, we found out, that the staff working in emergency belives to have all the knowledge needed to perform properly CPR and evaluates its work in such a predicament. We can say there is a gap between the operators considerings and the result of the evaluation of their performance. The solution which can be adapted depend on the economic availability and the local labor organisation. In bibliography they consider following aspects regarding the improvement of staff efficiencies in CPR. The retraining frequency should take into account the number of $\mathrm{CPR} /$ year carried out, the use of audiovisual system may allow a self-merit rating of their own work, improving the learning. Arranging an accurate feedback system for the staff, which shows detailed reports on the performed operations and also on the outcome of the patients, makes error identifying easier and prevents recurrences. Using these implements to integrate the training may also help to reduce costs

\section{Characteristics of patients receiving vasopressors}

Julie Benbenishty, Israel

Email: juliebenben@gmail.com

Patients receiving intensive care frequently need pharmacologic support of their blood pressure because of shock. In some patients, 


\section{* European Critical Care Nursing: Working Together for a Better Tomorrow.}

2011 congress abstracts *

shock is so severe that extremely high doses of vasopressors are needed to elevate their blood pressure. OBJECTIVE: We sought to ascertain the maximal dose of vasopressors administered to patients, and to describe the population of patients receiving vasopressors in one intensive care unit. METHODS: All adult patients admitted in 2001 to a 10-bed surgical unit in a university hospital, and receiving a vasopressor agent for 1 hour or more, underwent recordings of their demographic data, diagnoses upon admission, Acute Physiological and Chronic Health Evaluation (APACHE) II scores, vasopressors (including type, initial dose, dose increases, and maximal dose), number of days administered, complications, and mortality. RESULTS: Of 689 patients whose charts were reviewed, 72 received vasopressors. The mean age was 65 years, and $66 \%$ were male. The mean APACHE II scores were 24. The administration of $0.5 \mathrm{mg} / \mathrm{kg} /$ minute of norepinephrine or epinephrine resulted in $96 \%$ sensitivity and a specificity of $76 \%$ for the likelihood of mortality. Using Kaplan-Meyer curves, those patients receiving less than 0.5 $\mathrm{mg} / \mathrm{kg} /$ minute demonstrated an $80 \%$ 6-year survival. All 17 patients receiving more than $3.8 \mathrm{mg} / \mathrm{kg} /$ minute of norepinephrine, and all 5 patients receiving more than $9.6 \mathrm{mg} / \mathrm{kg} /$ minute of epinephrine, died. The length of time during which patients received less than their maximal dose of vasopressors had no influence on survival $(P=.4)$. The elderly (aged 75 years) and the young (aged $<75$ years) had the same intensive care unit survival rates when receiving vasopressors. CONCLUSION: In this study, little likelihood of intensive care unit survival was evident when patients received more than $.5 \mathrm{mg} / \mathrm{kg} /$ minute of norepinephrine or epinephrine. This study was an attempt by the nursing staff of one ICU to change non evidence-based practice.

Predictors of vascular complications post percutaneous coronary interventions

\section{Yardena Drori, Israel \\ Email: drori_ya@clalit.org.il}

Vascular complications are the most common adverse events after percutaneous coronary intervention $(\mathrm{PCl})$. Both cardiologists and nurses play a vital role in identification of risk factors and implementation of methods to reduce complications. Objectives: To assess predictors of vascular complications post PCI. Methods: Single center prospective study conducted by the nursing stuff with collaboration of cardiologists. Primary endpoint: Hematoma $>5 \mathrm{~cm}$, pseudo-aneurysm or AV fistula during hospitalization and 1-week after. A multivariable Cox proportional hazards model was used to evaluate the association between variables and outcomes. Results: 300 patients, mean age $59.1+10.9$ years, $20 \%$ females. Indications for PCl: 209 (69.7\%) acute coronary syndrome (ACS) (59 primary $\mathrm{PCl}$ ) and $91(30.3 \%)$ stable angina. Pretreatment: Aspirin and clopidogrel $262(87.3 \%)$, Enoxaparin or heparin $128(42.7 \%)$, llb/ IIlaGP antagonistis 50(16.7\%). Access site: $227(75.7 \%)$ radial/ulnar and $73(24.3 \%)$ femoral. All patients received weight adjusted heparin during catheterization. Primary endpoint was observed in 24 patients $(8 \%)$ : hematomas $>5 \mathrm{~cm}, \mathrm{n}=21$; pseudoaneurysm, $\mathrm{n}=3$; fistulas, $n=0$. Pseudoaneurysm, $n=3$ ( 2 needed intervention) and blood transfusion, $n=2$, were observed in femoral access only. Multivariate model, using patient characteristics, medications, interventional and hemostasis techniques, revealed that females (OR $1.13,95 \% \mathrm{Cl}$ 1.00-1.27, $\mathrm{P}=0.021$ ), ACS (OR 1.04, 95\% Cl 0.97-1.11, $\mathrm{P}=0.016$ ), femoral access (OR 1.26, 95\% Cl 1.06-1.50, $\mathrm{P}=0.007$ ) and pain during sheath insertion (OR 1.23, 95\% Cl 1.05-1.44, $\mathrm{P}=0.009$ ), were significant predictors of vascular complications. Conclusion: The main strategy to reduce vascular complications is the radial approach. Females, ACS patients and access site pain, were found to be at increased risk for vascular complications and need a careful follow-up.

\section{Induced hypothermia:the nursing care.}

\section{Drago Satosek, Suzana Rozman, Slovenia}

\section{Email: drago.satosek@kclj.si}

Background: The most common cause of sudden cardiac arrest is coronary artery disease. More than $50 \%$ of the patients die before reaching the hospital and at discharge survival rates remain as low as $5 \%$. Hypothermia has been demonstrated to decrease post resuscitation brain injury and improve the survival in comatose patients. . AIM: The aim is to develop nursing profession and standards for induced hypothermia, ensuring quality holistic nursing care and best teams. Results: Between the years $2003-2008$, 466 consecutive patients were admitted after resuscitated sudden cardiac arrest including 359 comatose survivors (77\%). Hypothermia was induced in $68 \% .57 \%$ of patients survived and majority survived with good neurological outcome (cerebral performance category 1,2). The nursing practice has improved through those years, especially the time management and efficiency of reaching the target body temperature. Implication for Practice: The results showed that good strategy and nursing care may improve survival with good neurological recovery. The nursing role in process of lowering patient's body temperature is very important. The target body temperature of $32^{\circ} \mathrm{C}-34^{\circ} \mathrm{C}$ should be reached as soon as possible, and should be maintained for 24 hours. Nurse should be aware of all side effects, complications and physiological signs and promptly react in case of adverse events. Education and training of critical care nurses play an important role. References: Knafelj R et al. Primary percutaneous coronary intervention and mild induced hypothermia in comatose survivors of ventricular fibrilation with STelevation acute myocardial infarction. RESUSCITATION, Volume 74, Issue 2, august 2007; 227-234. Kozik T M. Induced Hypothermia for patients with Cardiac arrest. Critical Care Nurse, 2007; 27: 36-42.

Antibiotic prophylaxis in device implantation: an observational study

\section{Luana Gattafoni, Italy}

\section{Email: luanagattafoni@virgilio.it}

Aim. Antibiotic prophylaxis has proved to reduce significantly infective complications related to pacemaker implantations 1 . Infections are $0.7 \%$ at implantation and $2 \%$ at replacement in large series 2 . From January 2004 up to January 2010 a prospective observational study for the prevention of infective complications was undertaken at our centre on all device (pacemaker or cardioverter-defibrillator) surgical procedures. Methods. All consecutive patients undergoing device surgery were administered cephazoline $2 \mathrm{~g}$ at the time of surgery and $2 \mathrm{~g}$ in the day following implantation. Clindamycine $1200 \mathrm{mg}$ at surgery and in the day following implantation was given to patients known or suspected to be penicillin-allergic. Patients undergoing device replacement were operated on a day-hospital regimen, hence received a single antibiotic administration at the time of surgery. Gentamycine $2 \mathrm{mg} / \mathrm{kg}$ was added at surgery in patients with mechanical heart valves or deemed at high risk for bacterial endocarditis. Vancomycine adjusted for renal function was preferred for patients with a greater than 10 days hospital stay before device implantation. All the patients had the pocket flushed with clindamycine $1200 \mathrm{mg}$ at skin closure. Results. In this 6-years period 2567 devices were implanted, 1630 pacemakers (1115 implants and 515 replacements), and 937 cardioverter-defibrillators (670 implants and 267 replacements). Cephazoline was administered to 2522/2567 $(98.2 \%)$ patients, clindamycine was administered to $41 / 2567$ (1.6\%) patients, and vancomycine to $4 / 2567(0.2 \%)$ patients. Five patients had pocket infections: $2 / 1785(0.1 \%)$ device implants, and $3 / 782$ $(0.38 \%)$ device replacements. A cutaneous rash developed in 2 patients $(0.1 \%)$ following cephazoline administration; no other effects related to antibiotics were observed. Conclusion. Our observational 
study compares favourably with former experiences $1-3$, the infection rate being 6 -fold and 5 -fold lower respectively at device implantation or replacement. More intensive antibiotic prophylaxis warrants a significant decrease of infections after device surgery.

The association between attitudes towards family-centered care and family presence during resuscitation among Israeli ICU nurses

\section{Freda DeKeyser Ganz, Faina Yoffe, Israel}

Email: freda@hadassah.org.il

Background: Family Centered Care (FCC) has been cited as important to patient care(1) however little is known about nurses' perspectives towards FCC. Family presence during resuscitation (FPDR) is an example of the implementation of $\operatorname{FCC}(2)$, however nurses do not necessarily agree with FPDR, especially those from non-western countries(3). It is unknown whether there is an association between FPDR and FCC. Aims: To determine: a. the attitudes of nurses towards FCC and FPDR $b$. whether there is an association between FCC and FPDR. Methods: A convenience sample of 96 Israeli ICU RNs completed 5 questionnaires: demographic data questionnaire; Nursing Activities for Communication with Families-Revised ;Barriers to Providing Family Centered Care-Revised ; Nurses' Experiences of Family Witnessed Resuscitation; and Attitudes to Family Presence during Resuscitation. Results: The item mean for the NACF-R and the Barriers scales were only 3.7 out of 5 and a moderate 2.4 out of 4 , respectively. Only 19 RNs (20\%) had experienced FPDR, of which 17 reported a negative experience. Overall, nurses objected to FPDR (mean item score $=1.8$ out of 5 ). No statistically significant relationship was found between FCC and FPDR. A significant negative correlation was found between the Barriers scale and FPDR $(r=-.36, p=.0001)$. Conclusion: While FCC has moderate support, objection still remains to FPDR. FPDR has been used as an example of FCC, but in this sample this might not be applicable. Increased education and policy changes should be encouraged to promote FCC and FPDR. 1.Henneman, Cardin . Crit Care Nurs. 2002; 22 (6):12-19. 2.Fulbrook, Albarran, Latour Int J Nurs Stud. 2005; 42: 557-568. 3.Gunes , Zaybak J Clin Nurs. 2009; 18:2907-2915.

\section{SESSION 2: END-OF-LIFE CARE}

\section{How do children die in Europe: the EURYDICE II study}

\section{Jos Latour, Netherlands and Denis Devictor, France}

\section{Netherlands, Email: jos.latour@planet.nl}

Aim: To investigate how end-of-life decisions are made in European Pediatric Intensive Care Units (PICUs). Methods: A multicenter, prospective study among 48 PICUs: 20 in Northern Europe (group 1); 5 in Eastern/Central Europe (group 2); and 23 in Southern Europe group 3. Data were collected between November 2009 and April 2010 using a survey method. Results: End of life decisions were made in $166(40 \%)$ of 412 deceased children (group 1: 72, group 2: 18 , group 36 ). In group 2, more patients died after CPR failure than after forgoing life support ( $p<.0001)$. Forgoing life support was mostly initiated due to poor neurological prognosis. In all PICUs, caregivers discussed the decision to forgo life support during a formal meeting after which the medical staff made the final decision. In $95 \%$ of the cases the decision to forego lif support had been documented in the medical record. Parents were more often informed about the formal meeting in group $3(p=.007)$. Parents were informed of the final decision in $96 \%$ of the cases (group 1: $97 \%$, group 2: $89 \%$, group 3: $97 \%$; $=.024)$. In $90 \%$ of the cases, the parents were at the bedside during their child's death. Conclusions: The results of this study and comparison with previous studies show a trend towards standardization of end-of-life practices across Europe in the past

\section{decade.}

\section{Is the ICU a proper place for end-of-life care?}

\section{Isabell Fridh, Sweden}

Email: isabell.fridh@fhs.gu.se

Aim The overall aim of the study was to explore and describe endof-life care (EOLC) in the ICU environment from the perspective of close relatives and nurses. Method Both quantitative and qualitative methods have been used. Study I was a survey concerning Swedish ICUs physical environment and routines when caring for dying patients. Study II was a study of the circumstances in conjunction with 192 patients' death in 10 ICUs. In Study III, interviews were conducted with 17 close relatives of 15 patients who died in three ICUs. The interviews were analysed using a phenomenologicalhermeneutic method. In Study IV, nine intensive care nurses were interviewed about their experiences and perceptions of caring for dying patients. Conventional content analysis was used. Results and conclusion The study showed that a great many ICU patients die after a short stay in the ICU, in multi-bed rooms and without the bedside presence of a next-of-kin. For close relatives, the experience of the EOLC provided in an ICU can be experienced as positive if the use of high tech is combined with compassionate and well-structured nursing care. In these circumstances, the care can be perceived as a confirmation that the loved one received the best nursing and medical care and that everything possible was done to save his or her life. Although close relatives seldom complain about the physical environment of the ICU, they want to be close by, and not separated from their dying loved one. From a nursing perspective, "doing one's utmost" is a decisive factor in EOLC in the intensive care environment. Key words: Intensive care, end-of-life care, physical environment

The views of ICU nurses on end-of-life care in public sector ICUs

\section{Shelley Schmollgruber, Gayle Langley, South Africa}

Email: shelley.schmollgruber@wits.ac.za

Despite advances in intensive care practice and technology, many patients die in these settings. Consequently, end of life care (ELOC) is common practice in intensive care units (ICUs) (Wunsch, Harrison et al, 2005: 823-931). However, ELOC practices vary across intensive care units and differences of nurses' involvement in this area remain (Latour, Albarran and Fulbrook, 2009). Attempts to improve ELOC in Europe by developing consensus-wide statements were developed. A prospective, descriptive study (The VENICE study) investigated whether nurses in ICUs had been influenced by the developments (Latour, Albarran and Fulbrook, 2009). This study, a replication of the VENICE study in the South African context, aimed to describe similarities and differences towards EOLC among intensive care nurses and to elicit nurses' involvement in EOL decisions in academic affiliated, tertiary/quaternary care hospitals in the Johannesburg area. The results are compared with those of the European study. A thirty question instrument developed by Latour, Albarran and Fulbrook and tested in the European context was adapted for the South African context. Eighty $(n=80)$ anonymous completed responses have been received to date from nurses working in the various intensive care units of Johannesburg public first level ICUs. The results are currently being analysed by means of descriptive and comparative statistics and will be presented in graphic and tabular format. Recommendations will be made for clinical practice and education of intensive care nurses. Key words: attitudes, decision making, end of life care, ethics, intensive care, nursing. 


\section{Withdrawal of therapy: how is the cooperation?}

\section{Hanne Irene Jensen, Jette Ammentorp, Helle Ørding, Denmark}

Email: hanne.irene.jensen@slb.regionsyddanmark.dk

Aim: The objective of this study was to examine intensive care nurses, residents, and attending physicians views on when withholding or withdrawing therapy should be considered and how cooperation regarding withholding or withdrawing decisions works. Methods: A questionnaire survey about different aspects of cooperation in correlation with withholding or withdrawing therapy at the intensive care unit (ICU). The study was conducted in seven hospitals (10 ICUs) in the Region of Southern Denmark. The ICUs were six general regional ICUs and four university ICUs. Results: A total of 495 nurses, 135 residents and 147 attending physicians participated in the study. The attending physicians came from two of the regional hospitals. The response rate was $83 \%$ ( $86 \%$ for nurses, $77 \%$ for residents, $82 \%$ for attending physicians). All three groups found that "Therapy futile" and "Wish of patient" were the two main reasons to consider withholding or withdrawing therapy. Attending physicians were most satisfied with the cooperation as $64 \%$ found their general experience of cooperation extremely or very satisfactory compared to $36 \%$ of the residents and $27 \%$ of the nurses. A total of $71 \%$ of nurses, $58 \%$ of residents and $32 \%$ of attending physicians thought that nurses should always or very often be involved in the decision-making process regarding withholding or withdrawing therapy, and $45 \%$ of nurses, $52 \%$ of residents and $38 \%$ of attending physicians found that nurses in practice were involved in the process. Additionally $43 \%$ of nurses, $29 \%$ of residents and $2 \%$ of attending physicians found that decisions regarding withdrawing therapy often, very often or always were postponed unnecessary. Conclusion: Nurses, residents and attending physicians differed in their opinion of the cooperation regarding withholding or withdrawing therapy practices at the ICU where attending physicians were the most and nurses the least satisfied.

\section{Taking patients home to die from intensive care}

\section{Laura Lockwood, Kate Gray, Sally Goodwin, United Kingdom}

Email: Llock1982@hotmail.co.uk

Facilitating a dignified death is an important part of Intensive Care nursing. Patient choice means that some patients want to die at home, in our intensive Care Unit, the Multi-disciplinary team have embraced this idea and made it possible. This presentation will detail our experience of taking a patient home to die. We will how we have made this possible by addressing the following points. Why offering this service is important to us. How we communicated with the families that we may be able to make this possible. What practical issues patients would need to consider before this could happen. What primary care services could be called upon and how we communicated with them. What legal issues we needed to consider, particularly the problem of who is responsible for what. Planning and implementation. Concerns about withdrawing treatment away from the security of the Intensive Care Unit and how this has worked in practice.

\section{Exploring new ways to deal with end-of-life}

\section{Julie Benbenishty, Maureen Ben Nun, Israel}

Email: juliebenben@gmail.com

It has been shown in the literature that ICU nurses are distressed by end of life treatment administered to patients today. Nurses are not only distressed by their feelings of failure, personal barriers and professional obstacles, but also by lack of educational resources offered to them. It is important to close the gap between what we know that needs to be done with patients approaching end of life, and how nursing staff can overcome emotional barriers. Nurses often follow end of life guidelines that they might accept cognitively, but find unbearably hard, because of emotional difficulty. Even with the best of intentions, the most updated knowledge of palliative care, supporting the patient and his family at the end of life is very difficult. Still today, there is a feeling of failure when the ICU team needs to stop fighting for a patient. A patient that is approaching end of life needs a dramatic shift of treatment emphasis. Many times the patient and the family need the acceptance by the staff of their end of life decision. Nurses as well as patients and families need a process of closure so that the end of life pathway is satisfying for all. In order to help nursing staff cope better with end of life situations, offering them evidence based guidelines, protocols and published articles is not enough. It is important to let nursing staff confront their emotions by way of workshops that allow the nursing staff to articulate their feelings, be aware of their value system and relate their reactions to former experience. It is especially important in a multicultural environment, where spiritual needs at EOL are versatile. We propose to offer an end of life interactive workshop for ICU nurses offering spiritual and communication tools enhancing end of life ICU care.

\section{Session 3: EDUCATION AND LEARNING}

\section{Student supervision in ICU}

\section{Evy Gundersen, Britt Sætre Hansen, Gunhild B. Bjørnå, Norway} Email: evy.gundersen@uis.no

The aim was to identify Intensive and Critical Care Nurses' (ICCNs) perceptions of supervising postgraduate students in ICU. Methodology The study was conducted in a 12-bedded general ICU in south west of Norway. Multistage focus groups were used to give the research participants a forum for discussing their perceptions of the supervisor role. The focus groups consisted of 8 ICCNs with variation in age and ICU experience. All of them were responsible of supervising students in a postgraduate critical care nursing program. The interviews were recorded, transcribed by the authors and analyzed by the use of qualitative content analysis. Preliminary results The ICCNs perceived their supervisor role as very demanding and time-consuming as the learning environment is very complex. The feeling of responsibility was sometimes accompanied by the feeling of loneliness, and the ICCNs valued constructive feedback from other nurses concerning the students. They also wanted the leaders to acknowledge this responsibility by giving them enough time to prepare and evaluate student supervision. They suggested establishing a supervision- resource group where the members were experienced student- supervisors aiming to educate all staff in supervision and reflection to better support students and colleagues in their development. They experienced that supervising students made them better ICCNs, and wanted to share this knowledge with their colleagues. They perceived their role as ICCNs and supervisors as very challenging, important and inspiring. Preliminary conclusion Loneliness, responsibility, lack of feedback as well as lack of system- thinking was perceived as problematic. The participants highlighted the importance of nursing leaders. Time and resources need to be allocated to improve student supervision in ICU, as the supervision process stimulates both students and supervisors to reflect and improve. References 1. Morgan, D.L. The Focus Groups as Qualitative Research, 1997, Sage Publication 


\section{Evaluating the mentor in critical care}

\section{Jochen Bergs, Marc Schuermans, Johan Mertens, Marc Rohaert, Belgium}

Email: jochen.bergs@uzleuven.be

Background: Hospitals need nurses and new nurses need guidance and support. They need this support to acquire their new role as professional nurses. When they do not succeed in this role transition; job satisfaction, performance and long term engagement are threatened. Mentor programs seem to provide the necessary guidance and support. Aim To evaluate the mentor program used in the department of critical care of the Leuven University Hospitals, Belgium. Methods Nurses who completed the 2009 mentor program were asked to fill in a questionnaire. The eight items were expected to yield specific information about the mentoring experience. The comments were analysed according to each survey item and themes previously determined to be important for successful mentorship: satisfaction, guidance and support, socialisation, match mentormentee, meeting with mentor and role model. In addition all comments were provided with a positive or negative code. Results Global satisfaction was good ( $78.4 \%$ positive comments) all nurses recommend there mentor for a future programme. Most positive comments are situated in the themes guidance and support $(32.7 \%)$ and satisfaction (23.5\%). Negative coded comments were dominated by the meeting with mentor $(59.3 \%)$ and guidance and support $(22.2 \%)$ theme. These comments are the result of insufficient stress reduction, feedback style and the need to spend more time with their mentor. Implications for practice Mentors need to be aware of the fact that new nurses experience stress even with good mentoring. The feedback style of the mentor determines the mentor experience of the mentee. More effort needs to be made to support the meeting opportunities between mentor and mentee.

A new approach to learn and teach intensive and anesthesia care in North Rhine-Westphalia

\section{Matthias Grünewald, Lothar Ullrich, Germany \\ Email: gruenewald@med.uni-duesseldorf.de}

Aim: Report about a new regulation to learn and teach intensive and anesthesia care in North Rhine-Westphalia (Germany) which started in 2009. This paper describes the contentwise, politicoeducational and didactical development of a new regulation for postgraduate courses in intensive care and anesthesia nursing by the state of North Rhine-Westphalia (Germany) in 2009. In the past courses in intensive care and anesthesia nursing in germany are part of the vocational training system and are not linked to university degrees. The new approach allows to transfer ECTS-credits from the vocational education system to university courses. In the first step, after a short introduction to the specific structure of further education in intensive care and anesthesia nursing in germany, we present the primary objectives and the construction principles of the new curricular approach: modularity, orientation in learning situations and competencies as learning outcomes. In a second step an exemplarily curricular conversion of the new regulation is outlined. The temporal arrangement of the modules on the one hand and the contentwise, methodical and curricular concretizing of the modules in learning situations on the other hand are described and the content and structure of some modules are presented. Finally we discuss the approach to link learning outcomes and qualifications from the vocational training system to the university system with ECTS-credits in terms of the European qualification frame (EQF). In this way the new regulation for courses in intensive care and anesthesia nursing in germany guarantees that the graduates have the necessary qualifications to make a substantial and indispensable contribution for the health care of critical ill patients in our society in the future.
A new way? Critical care education in the future in Austria; a vision

\section{Karin Klas, Austria}

Email: karin.klas@gmx.at

The aim of this work is to line out a possible way for an improvement of the education of intensive care nurses in Austria in the future. An internet based research in behalf of educationsystems in the field of critical care nursing in german (Germany and Suisse) and english (Great Britain and USA) speaking foreign countries took place. Four exemplarily choosen education-concepts from theese countries had been screened and the results were base for a comparison of the Austrian education with those from these countries. In addition to this, nursing educators and experts from Germany, the Suisse and Austria had been interviewed and their opinion was adducted to confirm the results, for they were used as a base for a possible new educationdesign for Austria. Necessary legal, pedagogic-didactic and personnel changes for the implementation were outlined and contemplated. On base of all this, a concept for a completely new and international comparable education modul on an exemplary base for Austria was designed. At the moment an implementation of this new concept is not possible for some major changes particularly in the legal field and with general conditions would be needed. Nevertheless this work could be the fundament for a new Educationconcept on a tertiary level in Austria. References: EfCCNa (2007): Position Statement on workforce requirements within European Critical Care Nursing Williams, G.; et Al (2006): Consensus Forum: Worldwide Guidelines on the Critical Care Nursing Workforce and Education Standards; In: Critical Care Clinics; 22; S 393-406 Neubauer, P. (2008): Dünne Luft für Weiterbildung; Ist die Weiterbildung Intensivpflege überarbeitungsbedürftig?; In: Padua; 4. September

What's the problem with problem-based learning?

\section{Steven Pearce, Anna Jones, United Kingdom}

Email: spearc01@bucks.ac.uk

Background: Problem based learning (PBL) is an educational strategy whereby the student is presented with a trigger which then acts as a catalyst for learning. PBL is a popular approach to learning in health care and is supported by an extensive body of literature (Koh et al, 2008). The reality of the triggers or scenarios is crucial for PBL (Roberts and Ousey, 2004) and carefully designed scenarios demand a range of skills from the student including self directed learning, critical thinking, team work and the integration of newly acquired concepts into pre existing knowledge (Wells et al, 2009). Aim: To discuss the evaluation of PBL in post registration critical care courses at two UK universities. Results: Overall, the student feedback was encouraging, especially from those for whom PBL was a new experience. Implications for Practice: We suggest that PBL is a useful strategy to help combat part of the downside to a reduced amount of face to face teaching time which will be an inevitable reality emerging from the planned reduction to the post registration nurse education budget. References: Koh, G.C., Koo, H.E., Wong, M., Koh, D. (2008) The effects of problem based learning during medical school on physician competency: A systematic review. CMAJ 178 (1): 34-41. Roberts, D. and Ousey, K. (2004) Problem based learning:developing the triggers. Experiences from a first wave site. Nurse Education in Practice 4, 154-158 Wells, S., Warelow, P.J., Jackson, K.L. (2009) Problem based learning (PBL): A conundrum. Contemporary Nurse 33(2): 191-201 
Electronic assessment for critical care nursing education: an evaluation of the use of a summative online scenario based debate

\section{Suzanne Bench, Kate Brown, Philip Blake, United Kingdom Email: suzanne.bench@kcl.ac.uk}

Background: The use of electronic learning (e-learning) in nurse education is increasing and is encouraged by Higher Education Institutions in line with current recommendations (JICS, 2009). Alongside this, electronic assessment (e-assessment) methods offer numerous advantages for post qualification nursing education, yet there is little evidence of their application in general (Ripley et al, 2009), and no evidence of their use within critical care nursing education. Aims This paper details the development, implementation and evaluation of a project designed to embed e-assessment into a critical care nursing module for intensive care and high dependency nurses. The e-assessment consisted of a scenario, requiring online debate. Each student group was facilitated by an identified lecturer who provided formative support. A summative assessment of each student was undertaken using existing school marking criteria alongside e-assessment criteria developed specifically for purpose. Results and implications for practice Despite a range of technical, cultural and attitudinal challenges, evaluation of the implementation of this novel strategy found it to be an effective method of assessment for this module, when appropriate resources were available. Implications for enhancing intensive care nursing education and practice will be discussed in light of such conclusions. References 1. JISC (2007) Effective practice with e-assessment; an overview of technologies, policies and practice in further and higher education. Higher Education Funding Council for England (HEFCE). Available online at: www.jisc.ac.uk/ assessment.html. Accessed 2/8/2010 2. JISC (2009) Effective Practice in a Digital Age; A guide to technologyenhanced learning and teaching. Higher Education Funding Council for England (HEFCE) Available online at: www.jisc.ac.uk/assessment. $\mathrm{html}$. Accessed 2/8/2010 3. Ridgway J, McCusker S, Pead D (2004) Literature review of e-assessment, Futurelab, Bristol. Available online at: http://www.nestafuturelab.org/research/ reviews/1001.htm. Accessed 2/8/2010

Web-based resources for critical care education: results from a concise review

\section{Ruth Kleinpell, United States of America}

Email: Ruth_M_Kleinpell@rush.edu

Overview: Web-based instructional methods are often categorized as e-learning, or the use of internet technologies. While information on the general use of web based learning is available, limited information exists on resources for critical care education. Aim: To identify and evaluate web based resources for critical care education. Methods: A multi-level search strategy was utilized. Literature searches were conducted (from 1996 to August 31, 2010) using OVID-MEDLINE, PubMed, and Cumulative Index to Nursing and Allied Health Literature (CINAHL) with the terms "web based learning", "computer-assisted instruction", "e-learning", "critical care", "tutorials", "continuing education", "virtual learning", and "web-based education". The websites of U.S. and international critical care organizations were reviewed for the availability e-learning resources. Finally, internet searches and email queries to critical care medicine fellowship program directors and members of national and international acute/critical care listserves were conducted. Results: Of over 250 identified resources, 125 programs for critical care exist in a variety of e-learning formats including tutorials, self-directed learning modules, interactive case studies, webcasts, podcasts, and video enhanced programs. Respondents to listserve queries $(>100)$, and critical care medicine fellowship directors and advanced practice nursing educator email queries $(>50)$ identified the use of a number of these programs. Conclusions: E-learning is actively being integrated into critical care medicine and nursing training programs, for continuing medical education, and competency training purposes. Knowledge of available web based educational resources may enhance critical care practitioners' ongoing learning. References Einarson E et al. Studies in Health Techn \& Inform.2009;146:841 Liolios A. E-Learning 2008 ESICM yearbook Vol 34, (1) Springer Publ Gerkin KL et al. J Ns Staff Dev 2009;25:E8-E13.

\section{SESSION 4: STRESS MANAGEMENT AND WORKFORCE}

ICU tend and befriend vs. flight or fight: a 'new' approach to decreasing stress in the ICU

\section{Freda DeKeyser Ganz, Israel}

Email: freda@hadassah.org.il

Healthy work environments have been shown to be associated with better patient outcomes yet ICU nurses are known to work in a stressful environment1. The initial phase of the stress response has been called flight or fight. It has been shown that most of the research conducted to determine this response was conducted on males. A new mechanism associated with the response to stress coined, "tend and befriend" might be a more appropriate female response to stress as opposed to fight or flight. "Tending" is defined as quieting and caring for the offspring and blending into the environment in case of threat while "befriending" is the creation of social associations. These responses involve the hormone oxytocin and have been shown to be associated with maternal attachment and social support. These behaviors are seen as evolutionary adaptive to the species, as women who are pregnant and/or have young children cannot effectively fight or flee and need additional resources to protect themselves and their young from threat2. Actions can be taken by individual nurses and by nursing administration to decrease ICU stress using a tendand-befriend strategy. This presentation will discuss the psychophysiological stress response from the flight/flight vs. tend/befriend standpoints as well as strategies that can be used to decrease stress and support nursing practice. 1: Mealer ML, Shelton A, Rothbaum B, Moss M. Increased prevalence of post-traumatic stress disorder symptoms in critical care nurses. Amer J Resp Crit Care Med. 2007; 175: 693-697. 2: Taylor SE, Klein LC, Lewis BP et al. Biobehavioral responses to stress in females: tend-and-befriend, not fight-or-flight. Psych Rev. 2000;107: 411-429.

Burnout symptoms among Croatian and Slovenian intensive care nurses

\section{Adriano Friganovic, Marijana Zaja, Asja Jaklic, Croatia}

Email: adriano@hdmsarist.hr

Introduction: Burnout is defined as high levels of emotional exhaustion and depersonalization or low level of personal accomplishment. Burnout is progressive loss of idealism and energy as a result of frustration and stress. Burnout can be caused by work environment, workload, management style, interpersonal relationships or personal reasons. Aim: This study aimed to identify burnout symptoms among intensive care unit (ICU) nurses and to compare burnout symptoms between Croatian and Slovenian ICU nurses. The survey was supported by Croatian Nurses Society of Anesthesia, Reanimation, Intensive Care and Transfusion and Slovenian section of anesthesia, intensive care and transfusion. Methods: A questioner measuring burnout symptoms was sent to ICUs in all Croatian and Slovenian hospitals. Results: Response rate was 374 ICU nurses: 237 from Croatia and 137 from Slovenia. The results showed no significantly differences between nurses in Croatia and Slovenia in first three degrees of burnout. Significant differences occurred in the group of nurses who have all symptoms of burnout. In the Slovenian group 


\section{European Critical Care Nursing: Working Together for a Better Tomorrow.}

2011 congress abstracts *

$21,8 \%$ of ICU nurses experienced burnout compared to s $12,4 \%$ in the Croatian group. This was correlated to the number of years working in ICU; $57 \%$ of Slovenian group worked more than 10 years in the ICU compared to $51 \%$ of Croatian group. Conclusion: Degree of burnout depends of employment period in the ICU. The results of this survey might indicate the need of rotation of nursing staff every 5 to 10 years. However, unhappiness with working condition is also a factor which can increase risk for burnout. Safe working condition, good organization, and possibility for education are factors which can might decrease risk for burnout.

Hospital nurses' exposure to violence and it's correlation with psychological distress and level of function at work

\section{Naomi Farkash Fink, Barel O, Gaash T, Levz D, Leibowitz V, Krayim P, Israel}

Email: naomifarkash@gmail.com

Introduction: Violence in health care systems is increasing in the world. Literature suggests that of all health professions, nurses are at higher risk to be exposed to violence. However, due to underreporting, there is lack of data on the real extent of the phenomenon. Like previous studies few research studies in Israel focused on violence in emergency rooms. The Aim To find out the extent of hospital nurses' exposure to violence, and it's correlation with nurses' psychological distress \& level of function at work Method \& Tools A retrospective study, of a randomized representative sample of 167 nurses of 5 hospitals(2general,1 psychiatric,1 geriatric,1rehab), in the center of Israel, returned a self reported, 3 parts questionnaire, on: exposure to violence at work in previous year, psychological distress \& level of function at work following the violent event. Results $80 \%$ of nurses reported having been exposed to violence ,mostly verbal abuse or threats. Maternity, Geriatric \& Rehabilitation ward nurses were exposed the most whereas the ICU nurses the least exposed. $64 \%$ of the worst events occurred on evening shifts, $61 \%$ reported not having the knowledge or skills to deal with these events and $94 \%$ expressed the need for education \& training. A significant correlation was found between exposure to violence \& psychological distress $(p=0.015)$ and a negative correlation between psychological distress and function at work $(P<0.05)$. Conclusion Violence in hospital is common in all areas where nurses work. There is a correlation between exposure to violence \& psychological distress and psychological distress may lead to decrease in the nurse's function at work. Recommendations Develepment of: zero tolerance policy to any form of violence against staff, a plan of quick response \& support for staff in events of violence and an in service education on the prevention \& deal ing with workplace violence

\section{Emotional intelligence of critical care nurses in South Africa}

\section{Amanda Towell, Elzabe Nel, Ann Muller, South Africa}

Email: ajtowell@uj.ac.za

Background/objectives: Critical care nurses often look after three or more critically ill patients during a shift. The workload and emotional stress can lead to disharmony between body, mind and spirit of the nurse. Nurses with a high emotional intelligence have less emotional exhaustion, psychosomatic symptoms; they enjoy better emotional health, more satisfaction (both at work and at home) and have improved relationships with colleagues at work. The question arises what is the emotional intelligence of critical care nurses? Methods: A quantitative survey was conducted. The target population was registered nurses working in critical care units attending the Critical Care Congress $2009(\mathrm{~N}=380)$. Data was collected with the use of the Trait Emotional Intelligence Short Form (TEIQue-SF). The data was analysed using SPSS. Results: The sample $(n=220)$ was mainly a mature, female and professionally experienced group of registered nurses. They held a variety of job descriptions in critical care units.
Conclusion: Statistics indicated that the standard deviations were small and no aberrant aspects such as demographics skewed the findings. Finally, a factor analysis identified two main concepts.

Correlation of indexes measuring the nursing workload concerning how critical status and the outcome of certain patients in the ICU

\section{Nikolaos Stoufis, Bakali Antonia, Greece}

Email: stouf@in.gr

Aim: The purpose of the present study is the evaluation of the correlation of the load steps of the nursing care Nursing Activity Score and TISS-28 score, depending on the critical condition and the outcome of the patients that were admitted in the ICU of a public hospital in Athens Greece. For the calculation of the critical condition and the prediction to death of the patients the following indexes were chosen: APACHE II and SAPS II. Method: The sample of the study is consisted from 115 patients admitted in the ICU of a public hospital in Athens Greece from November 2009 to May 2010. The climax For the statistical process of the data the statistical package SPSS 17.0 for windows was used. Results: Patients with high nursing workload according to the NAS index have 2.32 times greater possibility to die in relation with the other group of patients $(p<0.001)$. Every rise of the average value of the index TISS- 28 by one unit corresponds in a rise of the nursing duration at about 0.51 days, $(p<0.001)$, whilst every rise of the average value of the index NAS by one unit corresponds in a lowering of the nursing duration at about 0.25 days $(p=0.001)$. Conclusions: The application of the indexes of measuring the nursing workload could be a very useful tool into the understanding of the dimensions of the nursing care in a highly demanding area. References: 1 . Miranda R, Moreno R. Nursing Staff in intensive care in Europe. The mismatch between planning and practice. Chest $1998,113: 752-758$ 2. Miranda RD et al, TISS working group. Nursing avtivities score. Crit Care Med 2003,31:374-382 Key words - Nursing workload - TISS-28 - NAS

\section{Support to critical care nurses in South Africa}

\section{Elzabe Nel, Amanda Towell, South Africa}

Email: ewnel@uj.ac.za

INTRODUCTION AND PROBLEM One of the most important conclusions of a research study conducted by the Human Science Research Council of South Africa was that junior intensive care nurses bear a heavy burden as a result of staff shortages in the Intensive Care Unit (ICU) and that they do not receive enough support from nursing service managers. ICU nurses regularly present with work related problems such as lack of motivation, frustration and job hopping. Are nurses in South Africa unhappy due to a lack of support? The question arises "How do nurses experience the support they receive in ICU?" METHODOLOGY The purpose is to explore and describe the support nurses receive in ICU's in South Africa. A qualitative, descriptive, explorative and contextual research design was followed. The target population was junior ICU nurses working in various hospitals in Gauteng province. A purposive sampling was done. The sample consisted of junior nurses, working in five different ICU's. Three focus group interviews were conducted with the question "Tell me about the support in the ICU?" Trustworthiness was ensured. RESULTS The data obtained from the interviews was divided into four categories. From these categories, guidelines were set to better the support for ICU nurses. 
The formation of a world-wide network of critical care nursing organisations.

\section{Ged Williams, Australia}

Email: ged_williams@health.qld.gov.au

Introduction: Prior to 2000, there were less than 25 known critical care nursing organisations (CCNOs) in the world. Communication and collaboration of critical care nurses internationally was very limited compared to what it is today. - Study Objectives This study is a synthesis of three separate but related studies of known national CCNOs and leaders in 2001, 2005 and 2009 to inform how best to prioritise and coordinate the activities of a world wide network of critical care nurses. - Methods An electronic (Word) survey was distributed in each study period to all known CCNOs of the world. Questions related to the role, purpose and expected outcomes of national and international CCNOs and how best to support the needs of CCNs globally. - Results In each of the study periods the number of representative countries participating went from 23 to 51 to 63 respectively. The data has informed the formation of three multinational CCNOs networks - WFCCN, FLECI and APACCN - and resulted in CCNOs and leaders working collaboratively on many activities including international conference and educational activities, international research studies, development of international practice guidelines, peer reviewed electronic journal articles and the formation of new national CCNOs in the developing world. • Conclusion "Never doubt that a small group of committed citizens can change the world. Indeed, it is the only thing that ever has". (Margaret Mead) (1) Williams G. et al. 2001. World Wide Overview of Critical Care Nursing Organisations and their Activities. International Nursing Review. 48, Dec: 208-217. http://wfccn.org/assets/world_overview. pdf (2) Williams G,et al. 2007. Critical Care Nursing Organisations and Activities - a second worldwide review. International Nursing Review. Vol 54. 151-159. http://wfccn.org/assets/world_review.pdf (3) Williams G,et al. 2010. Critical Care Nursing Organisations and Activities - a third worldwide review. Study complete, manuscript in production. 2010.

\section{SESSION 5: CREATIVITY IN CLINICAL PRACTICE}

Design and validation of a new rating scale (COMHON Index) to estimate the risk of pressure ulcer in patients attended in critical care units

Angel Cobos Vargas, Garófano Jerez, Juan Rafael, Guardia Mesa, Manuel Fernando, Carrasco Muriel, Celia, López Pérez, Francisca, Gonzalez R. Amanda R., Aranda Salcedo, Tomás, Spain

Email: angcobvar@yahoo.es

AIMS Design a new specific scale to estimate the risk of pressure ulcer in patients admitted to critical care units (CCU). Assess its reliability and validity. METHODS A Multicentre, cross-sectional study has been performed. After a pilot study with 60 patients, the COMHON Index (that evaluates the level of consciousness, mobility, hemodynamic, oxygenation and nutrition, specifically defined for CCUs patients) was applied by five trained observers to 496 patients admitted in CCUs of two three-level hospitals in Granada, Spain. Kappa index was calculated to assess interobserver reliability. Construct validity and internal consistency was assessed through factorial analysis using VARIMAX with Kaiser normalisation (rotation method), Cronbach alpha and Pearson Intraclass Correlation Coefficient. Two alternative scales(Braden and Norton) were used to assess criterion validity, and the usual parameters of internal and external validity were finally estimated for predictive validity. All estimations were obtained separately for each hospital. RESULTS Regarding interobserver reliability, the total scale yielded Kappa coefficients of $0.886(95 \% \mathrm{Cl} 0.828-0.944)$ and $0.930(95 \% \mathrm{Cl}$ 0.884-0.977) in each hospital. Taking each item separately, kappa values ranged from $0.813(95 \% \mathrm{Cl} 0.751-0.875)$ to $0.979(95 \%$ $\mathrm{Cl}$ 0.955-1.000). Cronbach alpha values were 0.723 and 0.796 . The concordance between $\mathrm{COMHON}$ Index and Braden scale reached kappa values of $0.738(95 \% \mathrm{Cl} 0,6571-0,8184)$ and 0.812 $(95 \% \mathrm{Cl} 0,7410-0,8836)$. The corresponding values for Norton scale were $0.721(95 \% \mathrm{Cl} 0,6504-0,7925)$ and $0.733(95 \% \mathrm{Cl}$ $0,6631-0,8034)$. Joining the results from the two hospitals, validity parameters were $88,2 \%$ for sensitivity, $79,2 \%$ for specificity, $40,0 \%$ for positive predictive value, and $97,7 \%$ for negative predictive value CONCLUSIONS The COMHON index has shown a high validity and reliability; it can be used to identify patients at CCU with risk of developing pressure ulcers. The scale is flexible and different cut-off points can be alternatively used in order to increase its sensitivity or specificity, depending on available resources.

\section{Reducing pressure damage in critical care}

\section{Alison Evans, United Kingdom}

Email: wnsame1@cardiff.ac.uk

Background: Reducing pressure damage in the critically ill is a nursing priority; ulcers are associated with a two to four fold increase in the risk of death and are painful and debilitating (Bo et al 2003, Langemo et al 2000). This project focused on the introduction of a checklist of interventions for managing vulnerable patients, 'the SKIN bundle' (Gibbons et al 2006), and a safety cross to measure pressure ulcer acquisition. Aim To reduce by $20 \%$ pressure ulcer acquisition. Results The introduction of the SKIN bundle in critical care UHL has been delivering measurable improvements in care delivery and patient outcomes. Patients are consistently receiving evidence based care as demonstrated by compliance with all aspects of the SKIN bundle which has improved from a baseline of $68 \%$ to $95 \%$. Outcomes have significantly improved. In the fourteen months since its introduction there have been thirteen hospital acquired pressure ulcers, representing a $43 \%$ reduction in harm caused by healthcare associated pressure damage. Implications for practice The SKIN bundle is a simple concept, understood by both carers and patients and can be adapted to each clinical area and patient demographic. It has been fundamental to this improvement project that both process and clinical outcomes were measured to ensure that the interventions were making real differences to patients. This project demonstrates that achieving reliability in care delivery can be realised by care bundling and outcome measurement. Bo M, et al (2003) Predictive factors of in-hospital mortality in older patients admitted to a medical ICU. Journal of the American Geriatrics Society 5 (4) 529:533. Gibbons et al (2006) Eliminating facility-acquired pressure ulcers at Ascension Health. Journal on Quality and Patient Safety 32(9). Langemo D et al (2000) The lived experience of having a pressure ulcer: a qualitative analysis. Advances in Skin and Wound Care 13 (5) $225: 235$.

\section{Use of tooth brush for oral care and ICU patients' satisfaction}

Adriano Friganovic, Danijela Prugovecki, Vedran Dumbovic, Boris Medved, Irena Pastuhovic, Croatia

Email: adriano@hdmsarist.hr

Introduction: Patients in intensive care units (ICU) have needs related to their health stability during the ICU stay. The oral status of critically ill patient is often suppressed by their medical conditions. In the past, the oral care was not recognized as an important nursing care. Today, oral care is becoming standard procedure in ICU. Aim: This study aims to verify the degree of patient satisfaction of using tooth brush as a procedure in ICU versus usually used techniques of oral care ( oral swabs). Methods: A questionnaire was developed with 10 items measuring specifically patient satisfaction on oral 


\section{* European Critical Care Nursing: Working Together for a Better Tomorrow.}

2011 congress abstracts *

care. The sample was 90 ICU patients of three intensive care units (30 patients per ICU). Results: Of the 90 respondents, 71 (79\%) of the patients indicate oral care as a very important procedure in the ICU. The results confirmed that the patients using tooth brush were more satisfied compared to those receiving usually ICU oral care techniques. Conclusion: The results of this survey demonstrate the importance of oral care in intensive care unit. Implementation of well designed oral care protocols can really improve patient satisfaction as well as reduce infections. Use of tooth brush as a standard procedure in the ICU is better for patients because they feel comfortable and its increase level of self-respect and self-esteem.

Influence of prone position in extracorporeal carbon dioxide removal device function

\section{Maria Jesús Frade Mera, Amelia Guirao Moya, Laura Vergara Diez, Nieves Fernandez Gaute, Diego Montes Gil, Esteban Molano Alvarez, Spain}

Email: outplant.barcelona2@amexbarcelo.com

Background: Woman of 46-year-old admitted to ICU for acute respiratory failure with criteria for ARDS and nosocomial pneumonia. Due to the presence of refractory hypoxemia and bilateral bronchopleural fistula, Extracorporeal Carbon Dioxide Removal Device (Novalung) was indicated to reduce and optimize hypercapnia ventilatory management. Aim Discover Novalung effect on ventilation, taking into account the patient's position. Describe evolution and specific care of a patient with Novalung. Results Stay in ICU 26days. MBP $82 \pm 9 \mathrm{mmHg}$, HR $110 \pm 6 \mathrm{bpm}, \mathrm{Cl} 3.2 \pm 0.8 \mathrm{~L} / \mathrm{min} / \mathrm{m} 2$, ELWI $33 \pm 4 \mathrm{ml}$. Continuous haemofiltration 13.2 days with a median extraction of $50 \mathrm{cc} / \mathrm{h}$. Norepinephrine dose $0.68 \pm 0.79 \mathrm{i} / \mathrm{kg} / \mathrm{min}$ during 15days. Mechanical Ventilation 25.4days with PO2 $59 \pm 13 \mathrm{mmHg}$, PCO2 $68 \pm 35 \mathrm{mmHg}$, SatO2 $85 \pm 12 \%$, PO2/FIO2 69 \pm 35 , tidal volume $389 \pm 141 \mathrm{cc}$. Novalung 13days, heparin dose $181.42 \pm 145 \mathrm{mUl} / \mathrm{Kg} /$ min, cephalin time $57.56 \pm 16.41 \mathrm{sec}$, O2 flow $7 \pm 31 / \mathrm{min}$, median blood flow $1020 \mathrm{cc} / \mathrm{h}$, interquartile range $874 \mathrm{cc} / \mathrm{h}$. Prone cycles 4 , duration $53 \pm 27$ horas. With Novalung the PCO2 decreased regardless of position $66 \pm 21: 56 \pm 9, p=0.005$. Tidal Volume $512 \pm 67: 267 \pm 72$, $p=0.0001$. Pressure control(PC) $30 \pm 1: 17 \pm 3, p=0.0001$, and positive end-expiratory pressure(PEEP) $15 \pm 2: 10 \pm 2, p=0.0001$. Blood flow as supine-prone position $1053 \pm 82: 113 \pm 112, p=0.001$. Implications for practice The device's small size implied the success of prone technique without complications. It was possible to apply protective ventilation with normocapnia without alterations in blood $\mathrm{pH}$. The device blood flow decreased significantly during prone position, but this decrease didn't affect the functionality of Novalung with an increase in the $\mathrm{O} 2$ flow. References Bein T, Weber F, Philipp A, Passer C, Pfeifer M, Schmid FX et al. A new pumpless extracorporeal interventional lung assist in critical hypoxemia/hypercapnia. Crit Care Med 2006;34:1372-77. Hommel M, Deja M, von Dossow V, Diemel $\mathrm{K}$, Heidenhain C, Spies C, et al. Bronchial fistulae in ARDS patients : management with an extracorporeal lung assist device. Eur Respir J 2008;32:1652-55. Gordon E. Innovation in technology Novalung iLa challenges for the field of critical care nursing. Dynamics. 2009;20:14-7.

\section{SESSION 6: PREVENTING SLEEP DISTURBANCES}

\section{Sleep of the critically ill and the influencing factors}

\section{Marita Ritmala-Castrén, Professor Helena Leino-Kilpi, Finland}

Email: marita.ritmala-castren@hus.fi

Aim The aim of this literature review was to analyze, what is known about the critically ill adult pa-tients' sleep and what factors influence it. Methods Systematic literature search was done from Ovid MEDLINE, CINAHL, an "All EBM Reviews", and PsycINFO databases about patients' sleep in an ICU, with words related to sleep, assessment and critical care. Structure of analysis was based on the Redeker's (2002) Multifactorial model of sleep during acute care recovery. Additional focused searches were done in the areas of the model that the first search produced no information about. Results 44 research articles regarding critically ill patients' sleep were found. Most research was done about the sleep quality in an ICU, which is proven to be poor. Patients' sleep is mostly light and fragmented. Sound peaks explain fewer than $30 \%$ of awakenings, and light is not disturbing at all. Of the patients' illnesses, at least sepsis prevents totally normal sleep pattern. Nursing care during the night has been documented to be plentiful. Also ventilator care influences patients' sleep. Several areas remain unexplored. No studies show how patients' age, gender, emotional stress, previous health status, primary sleep disorders, or illness symptoms such as pain, fatigue or dyspnea, actually affect their sleep during the ICU stay. Nor has it been studied how poor sleep during ICU stay affects patient outcomes, their functional status or quality of life. Conclusion Even though it is well shown that critically ill patients sleep poorly, the reasons for it remain ob-scure and diverse. In order to be able to improve the quality of patients' sleep, more research is needed on what affects patients' sleep and with what interventions could it be improved. References Redeker NS. \& Hedges C. 2002. Sleep during hospitalization and recovery after cardiac surgery. Journal of Cardiovascular Nursing. 17(1):56-68.

\section{Sleep disorders in ICU patient. What can we do?}

\section{Ilaria Cossu, Italy}

Email: ilaria.cossu@aniarti.it

Background: Sleep disorders are common in critically ill patients and may adversely affect clinical outcomes. Reasons for sleep deprivation appear to be multifactorial. Aim. Describe factors that impact on sleep in ICU, the consequences of sleep disruption and the possible recommendations for nursing practice to enhance sleep in the critically ill and improve the quality of their long of stay. Materials and methods: Formal and informal Review of the literature (pubmed, nursing Italian websites, nursing books) of old ten years, to delineate the phenomena. Results: Sleep disorders are caused by patient's underlying illness, medications, therapeutic or monitoring interventions, mechanical ventilation and the ICU environment and staff. Sleep deprivation can impair tissue repair and cellular immune function and thus potentially affect overall morbidity and mortality. Incorrect interventions to maintain circadian rhythms can lead to prolonged hospitalization, development of ICU psychosis, and longer healing time. Modification of environmental factors, work organization, minimising arousals due to nursing procedures, and use complementary therapies (Relaxing massage, music therapy, therapeutic touch) could help promote sleep in the critically ill. Conclusions: Increased awareness of the importance of sleep in critically ill patients has led to the development of a number of specific strategies to optimize sleep in the ICU Friese RS, Sleep and recovery from critical illness and injury: a review of theory, current practice and future directions. Critical Care Medicin 2008) DaiWai m. Olson et all. Quiete time: A nursing intervention to promote sleep in Neurocritical Care Units. American Journal of critical care (2001) Vol 10 (2), 74-78 Richards K et all. Use of complementary and alternative Therapies to promote sleep in critically ill patients. Crit Care Nuers Clin North Am. (2003) Sep; 15 (3):329-40

\section{Earplugs in postoperative and critical care.}

\section{Ulrika Tunebäck, Tommy Bengtsson, Sweden}

Email: ullistu@hotmail.com

Aim of study: The purpose of this pilot study was to examine whether earplugs could improve intensive care and post-operative 


\section{* European Critical Care Nursing: Working Together for a Better Tomorrow.}

2011 congress abstracts *

patients' perceived sleep quality. Method: Quasiexperimental, nonrandomized, interventional study. Twenty-two patients from two intensive care units, a cardiac intensive care unit and a post-surgical department participated. In the intervention group eleven patients spent one night with earplugs, and eleven patients that slept without earplugs formed control group. The patients self-estimated their sleep by using the Richards-Campbell Sleep Questionnaire (RCSQ). Results: Patients who slept with earplugs rated their sleep as better (median RCSQ sleep index $=81$ ) than the control group (median = 58 ), but the proportion of patients in intensive and post-operative care that can benefit from the potentially positive effects of earplugs was limited. Conclusion: As the number of patients included in the study was small and not randomized to intervention- or control group it is not possible to draw any conclusions whether earplugs improve sleep in postoperative or intensive care, but among participating patients did the patients that used earplugs perceive their sleep as better than the patients that slept without earplugs did.

\section{SESSION 7: TRIAGE AND TRANSPORT}

The new Tuscan triage decisional algorithms: ED nurses' point of view.

\section{Giovanni Becattini, Federico Armiento, Stefano Bambi, Marco Ruggeri, Italy}

Email: g.becattini@usl7.toscana.it

Aim: In 2008 a new set of evidence-based decision-making algorithms for nursing triage in all the emergency departments (EDs) of Tuscany Region was drafted and immediately implemented through dissemination of a pocket handbook and an education program constituted of basic and retraining triage courses for 717 nurses. The aim of this study was to investigate the point of view of the ED nurses about the quality and feasibility of the regional triage courses contents in accordance with the Tuscan guidelines. Method: we designed a multicentric survey with an anonymous 11 closed items questionnaire dedicated to the nurses working in 16 Tuscan EDs, who attended to basic or retraining triage courses during 2008 and 2009. The survey was carried out from 10-07-2009 to 12-072009. Results: the questionnaires distributed were $378(92.2 \%$ of nurses who attended to a triage course). 350 were filled (85.3\%). 14 questionnaires were excluded for lack of inclusion criteria. $63 . \%$ of respondents attended to the basic triage course. 146 nurses $(43.5 \%)$ considered the triage courses contents enough remarkable, and $50.3 \%$ agrees enough with the triage level proposed by the new decisional algorithms. $64 \%$ of respondents affirm to follow the regional decisional algorithms during the triage level assignation, but only $41.6 \%$ consults the handbook or the digital format before the priority level assignation. $49 \%$ of surveyed nurses sometimes assigns a different triage level from the regional guidelines indications for contingence needs. Conclusions: the majority of nurses sufficiently appreciated the new triage decisional algorithms and the related education program, but more efforts are needed to implement their availability and application in the clinical practice. References $\cdot B a m b i$ S, Ruggeri M, Lumini E, Becattini G "La metodologia del triage. Verso la standardizzazione degli algoritmi decisionali nel Pronto Soccorso". Salute e Territorio 2009;173:91-94 •Delibera Regione Toscana n. 446 del 31-03-2010

\section{Safer transfer for critical care patients}

\section{Joanna Coward, United Kingdom}

Email: joanna.coward@nth.nhs.uk

Background: There are occasions when transportation of the critically ill patient is necessary and the ICS (2002) identified that there is evidence that outcome of critically ill patients is improved by the use of dedicated transport teams. Critical Care Networks are responsible for the coordination and development of transfer services within a defined geographical area to facilitate this process. Within the last 6 months the South Durham \& Tees Valley and the North East \& Cumbria and Critical Care networks have merged to form the North of England Critical Care Network (NoECCN). This covers a geographical area of 7,000 square miles and a population of 2.9 million. The guidelines for transfer of the critically ill patient have been amalgamated to standardise practices within the region. Aim - To standardise practices for Critical Care transfer within the North East $\cdot$ To improve the quality and safety of Critical Care transfers $\cdot$ To have a multi-professional approach to education and training of the practitioners involved in the transfer procedure Results The NoECCN have worked closely with external organisations including North East Ambulance Service (NEAS) in the development of these guidelines and this has resulted in: $\bullet$ Safer practice for patients where transfers are performed in a timely manner $\cdot$ Comprehensive robust guidelines to include NHS and independent hospitals $\bullet A$ multi-professional approach to training and developing competent practitioners - The development of a robust online audit system to record transfer details and any incidents. Implications for practice The NoECCN has facilitated this cross organisational collaborative approach to training and developed a safe environment for critical care patient transportation. References Comprehensive Critical Care: A Review of Adult Critical Care Services (Department of Health, 2000) Guidelines for the Transport of the Critically III Patient. Intensive Care Society Standards and Guidelines (2002).

The need for sea level flights for the critically ill: need or dogma?

\section{David Quayle, Jonathan Warwick, United Kingdom}

Email: david.quayle@airmed.co.uk

This paper reviews the evidence relating to the requirement to transfer a critical care patient by air with a sea level equivalent cabin pressure. The authors will contextualise with an overview of the critical care aeromedical transport work undertaken by AirMed including the role of a senior critical care nurse (Chief Flight Nurse) within the organisation as the first line in reviewing patients and arranging flights. Fixed wing air ambulance flights are complicated by the stressors of flight which have major potential implications for the care of patients during air transport. The authors will recapitulate on these stressors with specific concentration on gas expansion at altitude, exploring a number of nursing and medical management issues. The usual indications for a sea level cabin altitude of pneumothorax, intraabdominal air, pneumocephalus and intra-occular air are explored, along with the potential complications that may be encountered at a higher altitude with each of these conditions. Also addressed are the disadvantages of sea level cabin altitude and the implications for nursing and medical management should one be undertaken. While the conditions explored are normally regarded as absolute indications for sea level transfer, the literature demonstrates that another safer and cheaper approach may be indicated which would make sea level cabin altitude flights a rarity. This would further enhance the quality of care available for the critically ill patient requiring air transfer.

\section{Management of a traumatic brain injury database}

\section{Gunilla Malmborg Bornhall, Sweden}

Email: gunilla.malmborg-bornhall@karolinska.se

Background: "To view the impact of our work, we need to measure our results"! The Traumatic Brain Injury (TBI) is a major cause of death an disability, therefore it is important to register and followup this patient group, and try to improve our treatments. The TBI database at the Neurosurgical clinic at Karolinska university hospital in Stockholm, Sweden, is a major database - the registration started already in the 60:ths century. Aim: A presentation to the management 


\section{* European Critical Care Nursing: Working Together for a Better Tomorrow.}

2011 congress abstracts *

of a TBI database, that hopefully attract others to begin register! Results: The TBI database contains over 130 different parameters on each registration, for example vital signs and treatment prehospital and at Karolinska. Assessment of Glasgow outcome scale (GOS) are registered at 3 occasions in the first year after the TBI. A follow-up by a letter, 1 year after the TBI, provides information about how the patients are capable living their everyday life. Data from this database can be investigated and used in many different ways - for example the patients outcome. Many of the patients has generally sequelae as memory deficits, fatigue, headache, damaged smell and taste function and concentration problems. Implications for practice: Management of a TBI database is time-consuming! Regular time set aside for the registration, is really important to be capable of register continuously. Easy availability to medical records, facilitates the flow in the work and saves time.

\section{SESSION 8: WORKSHOP}

Workshop - Development of a critical care nursing competency document: an EfCCNa project

David Waters, Anne Kokko, Drago Satosek, Heike Strunk, Evanthia Georgiou, Maria Hadjibalassi, EfCCNa

Email: david.waters@bucks.ac.uk

Background: The European Federation of Critical Care Nursing Associations (EfCCNa) aims to represent critical care nursing across Europe. The EfCCNa Education sub-committee was established to promote \& champion education issues within the Federation. In response to inconsistent and varied critical care nursing education provision across Europe (Ääri et al, 2008), the Education subcommittee has sought to develop a competency framework \& position statement. Aims: To explore the issue of competency frameworks within critical care nursing. To present ongoing project work \& provide an opportunity for dialogue and consultation. Structure of Session: Initially definitions of competence \& available frameworks within critical care nursing will be introduced. Key European \& international drivers for critical care competency will also be explored. The main aspect of this session will present an ongoing project by the EfCCNa Education Committee, focusing on the development of a critical care nursing competency document \& position statement. The competency domains/themes will be presented to the audience, with an opportunity for audience consultation, discussion and feedback. References: Ääri, R., Tarja, S. \& Helena, L. (2008) Competence in intensive and critical care nursing: a literature review. Intensive \& Critical Care Nursing. 24. 78-89.

\section{SESSION 9: RESPIRATORY CARE AND INTERVENTIONS}

Influence of nursing workload on the incidence of ventilatorassociated pneumonia

Francisco Javier Carmona Monge, Ana Jara Pérez, Sonia García Gómez, Montserrat Martínez Lareo, Gloria Rollán Rodríguez, Isabel Cerrillo González, Cristina Quirós Herranz, Rosana Alameda Varela, Rafael Ruiz de Luna, Spain

Email: Eli.Casado@smith-nephew.com

Background: Rutinary assessment of nursing workload is essential in critical care to assure patient safety and quality of care. There are few studies that analyze the existing relationship between nursing workload and the development of nosocomial infections. Ventilator associated pneumonia (VAP) is one of the adverse events that can be more easily avoided. The aim of this research is to analyze the relationship between nursing workload and ventilator associated pneumonia (VAP) in a medical ICU. Methods: correlational study performed in a 12 bed medical ICU (October 2007 - January 2009). Nursing workload was measured using the Nursing Activities Score. Data regarding mechanical ventilation and endotracheal aspirate cultures were recorded daily. Results: Median daily nursing workload during this period was 722.8 points (593.7-819.2). A total of 19 VAP were diagnosed during this period, being the incidence of 6.2 infections for 1000 days of mechanical ventilation. Pearson correlation between the number of new VAP diagnosed each day and total nursing workload in the ICU (total NAS score) in the previous 15 days to the diagnosis were calculated. A significant correlation was found between VAP and nursing workload on day 11 , 12,13 and 14 before the VAP onset was detected $(r=.094, p=0.042$; $r=.111, p=0.016 ; r=.130, p=0.005$ y $r=.136, p=0,003$, respectively). Conclusion: An increase in nursing workload in critical care can be a risk factor for the development of VAP. Reduction of VAP is essential to increase patient safety and to improve outcomes of critical care patients. Adequate staffing may have an important role in fulfilling this objective.

\section{Is humidification necessary?}

\section{Tiina Rintala, Helene Karlman, Sweden}

Email: helene.karlman@karolinska.se

Aim: Patients with neurological disorders treated in NICU often need ventilator treatment longer than other ICU patients. These patients are at greater risk of aspiration, due to lowered level of consciousness, which leads to lower respiratory tract infections. These lungkomplications often cause circulatory and neurological disorder such as increased intracranial pressure. Former studies show that active heated humidification decrease incidence of VAP and decreased hospital stay and time on ventilator. We wanted to see if active heated humidification could improve and ease the care of NICU patient's lungcomlications. Method 112 patients were included in the study; 41 patients received active heated humidification, 71 got customary treatment. Patients were randomly placed in the beds equipped with humidification system. Remaining patients in need of assisted ventilation received common treatment with HME-filter. The study went on for 1 year. Assessed parameters of interest were GCS on admission, PFI, nursing work load, APACHE II, time spent in NICU and time spent on ventilator. As a new routine airway cultures twice a week were introduced. Airway cultures, bronchoscopies and lower respiratory tract infections were registrated as amount of inhalations, suction of lower airways, colour and consistency of secretion. Results No differences were indicated concerning time spent in NICU or on ventilator. Slightly more lower respiratory tract infections occurred among patients with active heated humidification. Conclusion Since no improvements were seen concerning time spent in NICU or on ventilator, the unit are not going to use active heated humidification as a standard procedure. However benefits were noticed during suction of lower airways and bronchoscopies due to more loose secretion. References Kola et al. (2005) Intensive Care Medicine. 31 Lorente et al.(2006) Critical Care 10

Endotracheal suctioning in children: what is evidence based?

\section{Irene Harth, Germany}

Email: iharth@onlinehome.de

Aim: The aim of this lecture is to point out the current state of the art of endotracheal suctioning in paediatric patients and to mention procedures which are scientifically approved in order to divide these from the variety of pratices which still can be found in airway management in paediatric intensive care. Methods:A literature search in Pub Med and CINAHL was performed in order to find relevant papers on endotracheal suctioning in children. Results: 


\section{* European Critical Care Nursing: Working Together for a Better Tomorrow.}

2011 congress abstracts *

Most of the studies on endotracheal suctioning were performed in an adult population and only a few were available for the paediatric and neonatal clientele. Nevertheless there is clear evidence that endotracheal suctioning, although it is a common procedure in ventilated patients, is associated with potential risks. Regarding several aspects of endotracheal suctioning e.g. specific techniques, catheter sizes etc there are no clear recommendations available. Recruitment maneuvers should not be performed routinely after suctioning as it is not beneficial for the patient; preoxigenation may be indicated in specific group of patients and the instillation of isotonic saline solution prior to suctioning is seen as detrimental for patients. References: Suctioning Practices and Airway Management Practices: A Pilot Study and Instrument Evaluation. Mary Lou Sole et al. American Journal of Critical Care, July 2002 Vol. 11, Nr. 4 Endotracheal Suctioning with or without Instillation of Isotonic Sodium Chloride Solution in Critically III Children. Debra Ann Ridling et al. American Journal of Critical Care, May 2003 Vol. 12, Nr. 3 An evaluation of the implementation of a best practice guideline on tracheal suctioning in intensive care units. Janita Chau et al. International Journal of Evidence-Based Healthcare September 2007, Vol. 5 Nr. 3 A comprehensive review of pediatric endotracheal suctioning: Effects, indications, and clinical practice. Brenda Morrow et al. Pediatr Crit Care Med 2008 Vol 9, Nr. 5.

Routine versus on demand manual hyperinflation in cardiac surgical patients: a randomized controlled trial

Frederique Paulus, Denise. P Veelo, Selma B De Nijs, Paul Bresser, Ludo FM Beenen, Bas A de Mol, Jan M Binnekade, Marcus J Schultz, The Netherlands

Email: f.paulus@amc.uva.nl

Aim: Cardiac surgical patients show deterioration of functional residual capacity (FRC) after extubation. Manual hyperinflation prevents airway plugging, and as such could improve FRC. We performed a randomized controlled trial comparing a "routine" with "on demand" $\mathrm{MH}$ in cardiac surgical patients. Methods: Patients after elective cardiac surgery and admitted to the ICU of a university hospital were randomly allocated to "routine" (MH within $1 / 2$ hour after arrival in the ICU and every 6 hours until tracheal extubation) or "on demand" $\mathrm{MH}$ (MH only in case of failed endotracheal suctioning while sputum is obviously present, or in case of oxygen desaturation not responding to 3 minutes hyper-oxygenation) during mechanical ventilation. FRC was measured pre-operatively and 1, 3 , and 5 days after extubation. Peripheral hemoglobin saturation (Spo2) was measured during the first 3 days after extubation while the patient was breathing room air. Results: A total of 100 patients were enrolled. Patients in the "routine" group received median [IOR] 2 [2-3] MH procedures compared to 0 procedures in the "on demand" group. In both groups, FRC was significantly reduced after surgery. In the "routine" group FRC decreased to $72 \%$ of the preoperative measurement, versus $59 \%$ in the "on demand" $(P=0,002)$. Differences in FRC were statistically not significant at day 3 and 5 . Conclusion: "Routine" MH attenuates reduction of FRC in the early post-operative day after cardiac surgery.

What are critical care nurses' perceptions of the long-term mechanically ventilated patient in the ICU setting?

\section{Lisa Mace, United Kingdom}

Email: Lisa.Mace@UHBristol.nhs.uk

Aims of the study: This study aimed to explore ICU nurses experiences and attitudes of caring for long-term critically ill patients. The purpose of the study was to generate a detailed account of how nurses engaged and coped when dealing with patients who required a prolonged period of care, including artificial ventilation, in
ICU. Background: Previous research alluded to factors which make caring for long-term ICU patients difficult and challenging. There was also some evidence which suggested that particular patient types are 'unpopular' with ICU nurses. Design: Using a purposive sample, a qualitative design was utilised to unravel ICU nurses' perceptions of the long-term critically ill patient. Method: Twenty three critical care nurses, recruited from either the general or cardiac intensive care of one hospital trust, participated in one of four focus group interviews. The data generated was analysed using thematic content analysis. Findings: Long-term patients were identified as physically and emotionally demanding to care for a variety of reasons. Nurses in some instances felt ill prepared to deal with their complex needs. Balancing the needs of the long-term critically ill with those of the acutely ill was an ongoing organisational tension for senior staff. Conversely, long-term patients were viewed as producing rewarding and satisfying experiences of caring; more evident when ICU nurses felt they connected with individual patients. Conclusions The idea of the 'unpopular' patient still exists and is evident in the modern ICU setting. Preparing nurses to cope with the challenges which caring for the long-term critically ill pose through better support and additional education/training, will do much to maximise the care this patient group, while minimising the stress ICU nurses experience when caring for them. As a result, ICU nurses may come to perceive this patient population in a more positive light.

Survival in ALS patients with non-invasive and invasive home mechanical ventilation: 10 years of experience in west Denmark

\section{Pia Dreyer, Charlotte K Lorenzen, Lone Schou, Denmark}

\section{Email: piadreye@rm.dk}

Background: Patients suffering from Amyotrophic lateral sclerosis (ALS) have been offered non-invasive and invasive home mechanical ventilation (HMV) in west Denmark since about 1999. They represent a small but increasing patient group, and there has been a growing use of HMV in ALS both non-invasively and invasively. The use of HMV in ALS varies very much be-tween centres and countries, maybe depending on the clinician's attitudes and missing evidence based guidelines of when to start HMV. Only a few studies address the possible clinical effects and survival and the differences between bulbar ALS and spinal ALS. Objectives To study patients diagnosed with bulbar and spinal ALS according to survival and when noninvasive and/or invasive HMV were started. Method A descriptive retrospective study of all patients diagnosed with ALS cared for at Respira-tory Centre West in Denmark from 1998 to 2009 and treated with non-invasive and/or in-vasive HMV. Data-registration of time for diagnosis differentiated in bulbar and spinal ALS, initiation of noninvasive and/or invasive HMV until eventual death was recorded. Survival was analysed. Findings The data shows that there have been an increasing number of ALS patients admitted to Respiratory Centre West in Denmark. Retrospectively 301 patients with ALS were admitted to Respiratory Centre West in the period January 1998 to December 2009. 119 women and 182 men with a mean age of 61.5 (range 23-89). The mean treatment time in 161 patients related to non-invasive HMV was 11.5 month (range 0-104). The mean treatment time in 35 patients related to invasive HMV was 21 month (range 1-82). Conclusion Most surprisingly there was no difference in survival in the differentiated groups of spinal, bulbar and mixed ALS related to non-invasive and/or invasive HMV. 


\section{SESSION 10: DELIRIUM IN ICU}

Physical restraint use in ICUs across Europe: the PRICE study

Julie Benbenishty, Ruth Endacott, Shiela Adam, Israel, UK Email: juliebenben@gmail.com

The use of physical restraint has been linked to delirium in ICU patients and a range of physical and psychological outcomes in non-ICU patients. However the extent of restraint practice in ICUs is largely unknown. This study was designed to examine physical restraint practices across European ICUs. A prospective point prevalence survey was conducted in adult ICUs across European countries to examine: physical and chemical restraint use during the weekend and weekdays, reasons for physical restraint use, timing of restraint use, type of restraint used and availability of restraint policies. Thirty four general (adult) ICUs in nine countries participated in the study providing information on 669 patients with details of physical and chemical restraint use in 566 patients. Prevalence of physical restraint use in individual units ranged from $0-100 \%$ of patients. Thirty three per cent of patients were physically restrained; those that were restrained were more likely to be ventilated $\div 287.56$ $p<0.001)$, sedated $(\div 234.66 p<0.001)$, managed in a larger Unit $(\div 210.741, p=.005)$ and managed in a unit with a lower daytime nurse:patient ratio $(\div 217.17, p=0.001)$. Larger units were more likely to use commercial wrist restraints and smaller units were more likely to have a restraint policy, although these results did not reach significance. As an initial exploration, this study provides evidence of the range of restraint practice across Units in Europe. Variation in the number of units per country limits generalization of findings. However, further examination is needed to determine whether there is a causal element to these relationships. Attention should be paid to developing evidence based guidelines to underpin restraint practices.

\section{Interventions for preventing ICU delirium}

Thordis Thomsen, Ingrid Greve, Ingrid Egerod, Helle Svenningsen, Camilla Bekker Mortensen, Ann M. Møller, Denmark

Email: thotho02@heh.regionh.dk

Aim: To investigate the effect of designated ICU-delirium prevention interventions compared to standard ICU-practice and/or placebo on the incidence of ICU-delirium in adult ICU-patients. Methods: A systematic review ad modum Cochrane. Relevant databases will be searched for randomised controlled trials (RCT's) of ICU-delirium prevention interventions. Trial inclusion, risk of bias assessment and data extraction will be performed by three authors. Risk ratios (RR) for the risk of ICU-delirium will be calculated, and, if appropriate, pooled effects will be estimated using the fixed-effect method. Results: We are currently writing the protocol for the study. We expect to be able to present the protocol at the EfCCNa in the spring.

Recognising and improving outcomes for the delirious patient in ICU

\section{Linda Bower, United Kingdom}

Email: linda.bower@medway.nhs.uk

Background: Monitoring patients for signs of delirium is important and we decided to implement a delirium assessment scoring tool in our ICU. Research identifies delirious patients as having an increased risk of mortality (Ely, 2004), with associated costs such as increased ventilator days and length of stay. Aim: The aim was to introduce a validated scoring system to identify delirious patients. We recognised the hyperactive delirious patient in the ICU; however, we appeared only to manage these patients reactively. We were less able to identify the hypoactive delirious patient. Polderman and Smit,
(2005) recognised them as the most difficult to detect. In identifying the delirious patient we hoped that not only would we enhance their ICU journey but also improve mortality. In order to do this a way of educating nursing staff had to be decided upon. Results: Staff initially found difficulties scoring patients. Through education, staff recognised the benefits of monitoring for delirium. A 35\% incidence of delirium in our patients was found and there was an increase of $157 \%$ in ventilator days (5.9 - 2.3 days) and $91 \%$ increase in length of stay (9.29 - 4.86 days) for those identified delirious patients compared to the non-delirious patient. We have now developed a management plan for the delirious patients and the next steps are to ensure that this action plan conforms to the new NICE guidance. Ely, W. Shintani, A. Truman, B. Speroff, T. Gordon, S. M. Harrell, F. E. Inouye, S. K. Bernard, G.R. and Dittus, R. S. (2004) Delirium as a Predictor of Mortality in Mechanically Ventilated Patients in the Intensive Care Unit. Journal of America Medical Association, 291: 1753-176. NICE 2010 DELIRIUM: diagnosis, prevention and management CG103 NICE London Polderman, K, H. and Smit, E. (2005) Dealing with the delirium dilemma. Critical Care, 9(4), 335336.

Nationwide survey of sedation and delirium assessment at Danish ICUs

\section{Helle Svenningsen, Else Tønnesen, Denmark}

Email: hellsven@rm.dk

Critically ill patients who develop delirium have longer stay in intensive care unit (ICU) and increased morbidity and mortality. To reduce pain and discomfort from the necessary care and treatment these patients are often sedated. However sedation can especially in the elderly patients provoke delirium, and for all patients sedation can obscure delirium, due to the inactivity sedation causes. The international guidelines recommend systematic use of validated scales for assessment of sedation levels in ICU-patients To assess whether a patient has delirium, it is necessary to assess sedation level, since changes in this is one of the symptoms of delirium. The aim of this survey was to elucidate clinical practice around the assessment of sedation and symptoms of delirium in intensive care patients in Denmark. On the basis of the received results we discussed pros and cons of the different tools. Method: Questionnaire was in May 2009 via e-mail sent to the research network of the Professional Associa-tion of Anaesthesia, Intensive care and Recovery nurses (Faglig Sammenslutning af Anæstesi- Intensivog Opvågningssygeplejersker FSAIO) for the 46 ICUs who offered ventilator treatment to adults. An ICU-nurse with everyday-patientcontact was asked to report the department's practices around the sedation and delirium assessments in adults ICU-patients. Results: All 46 departments $(100 \%)$ responded either by returning a paper version of the questionnaire (12), by emailing the completed form (24), or by telephone (10). In almost all Danish ICUs patient sedation levels were assessed. There is no consensus on what sedation assessment score was used, or on how frequently and to which patient groups they should be used. Delirium assessment was performed occasionally, and rarely systematic. Only 5 departments assessed pa-tients systematically using CAM-ICU. Less than half of ICUs have guidelines for the treatment of delirium.

\section{Italian nurses's perception of delirium in ICU. A survey}

Silvia Scelsi, Piergentili Federica, Sansolino Sandra, Gian Domenico Giusti, Italy

Email: s_scelsi@yahoo.it

INTRODUCTION Delirium is a very common syndrome in ICU population. It's an acute disturbance of consciousness and cognition. A late assessment of this syndrome can lead to a longer ICU stay, prolonged mechanical ventilation and higher morbidy 


\section{* European Critical Care Nursing: Working Together for a Better Tomorrow.}

2011 congress abstracts *

e mortality. MATERIAL AND METHOD The aim of this study is assessing Italian nurses perception of delirium in ICU. Between October 2009 and January 2010 a survey was distributed to Italian ICU nurses. RESULTS 455 nurses responded to the survey. $54 \%$ considered delirium a problem in $25 \%$ of ICU patients. $50 \%$ of respondents don't know correlate this syndrome to ICU stays. $85 \%$ of respondents consider delirium a problem for their daily activities and they perceive sleep deprivation, absence of family members and alcohol/drug abuse as risk factors. A proper assessment tool was used only in $12 \%$ of Institute investigated. DISCUSSION Delirium is considered a great issue in Italian ICUs but it's also underestimated. The researcher didn't consider the design of ICUs, if open-space or single-bed rooms. Uncommon is the bedside presence of family or relatives to relief patients' confusion. CONCLUSION Italian nurses have a clear perception of delirium in ICU, unfortunately they cannot change nursing performances even if they increase delirium risk factors.

\section{Restraints in ICUs: a mixed method study}

\section{Gayle Langley, Anthony Egan, Shelley SchmollgIruber, South} Africa

\section{Email: Patricia.Apfel@wits.ac.za}

Summary: The use of mechanical restraints is a controversial practice internationally but is common in South African Intensive Care Units (ICUs). Their use was studied in the ICUs of an academic, public hospital in Johannesburg, South Africa. Research Methodology Quantitative data were collected over two months by means of a checklist and analysed by means of descriptive statistics. The patients' length of stay, type of restraint used and duration thereof, the daily nurse patient ratios, as well as information regarding sedation and analgesia were detailed. Individual interviews with twenty medical and nursing ICU clinicians elicited their stated rationale for restraint, their experiences and perceptions of the practice and recommendations for restraint. Findings Of a total of 219 patients in the three ICUs, 106 (48.4\%) were restrained. The average number of days restrained was nine with a range of days from 1 to 53 (in one case only). In only six cases were restraints other than wrist ties used. Forty seven of the restrained patients had sedation and analgesic medication prescribed and 59 not. All participants conceded a place for physical restraint in the ICU, primarily to ensure the safety of patients but were divided as to the reason for restraining patients and disputed the benefits of restraint. Conclusion Poor communication between the team, patients and families and diminished collaboration within the multi-disciplinary team emerged as central concepts in this study and influenced care decisions and practices regarding the use of mechanical restraints in the clinical area. Key Words: Intensive Care Units Mechanical restraints Team Communication Team Collaboration

\section{SESSION 11: PROTOCOLS AND STANDARDS}

\section{Mobilisation of obese ICU patients: a clinical study}

\section{Pia Dreyer, Nis Kaasby, Rikke Højbjerg, Denmark}

\section{Email: piadreye@rm.dk}

Background: The number of obese patients in Intensive care units (ICU) is increasing. This raises spe-cial demands on the staff, the surroundings and the equipment. Since 2007 mobilisation of obese ICU patients has been a clinical study in our ICU, containing three studies until now. Aim 1) to investigate whether obese ICU patients $(\mathrm{BMI}>40)$ are mobilised according to the clinical standard, 2) to investigate the healthcare professionals experiences of working with mobilisation of obese ICU patients and 3) to make practical use of staff member's innova-tive thinking. Methods 1) A retrospective observational study (2007-2008). Focus group interviews of the staff in two qualitative studies: 2) with focus on experiences (2009-2010) and 3) with focus on so-lutions, clinical guidelines and checklists within the themes conducted in study two (2010-2011). Results 1) Seventeen patients with $\mathrm{BMI}>40$ were included. Two were mobilised according to the clinical standard. The average mobilisation was $2.87 /$ day (range $0-6$ ) for the remaining 15 patients. 2) The analysis of four focus group interviews showed that it is more difficult to care for the obese patient, for reasons such as lack of space, non-availability of the correct equipment and available staff. Furthermore the attitude of the staff plays a significant role in the treatment and care of the obese patient. 3) The analysis of focus group interviews showed that local guidelines are needed for how to organise most available space and a sufficient number of staff, education in handling equipment (bariatric bed, bariatric sling, lift-systems) and changing attitude (the good story). Study three is work in progress. Conclusion Only two of 17 patients were mobilised according to the clinical standard. Space, equip-ment and available staff members are needed if the clinical standard for mobilisation of the obese ICU patients has to be met.

\section{Transpyloric feeding in major burns}

David Carbajales, Monica Soler Rovira, Teresa Pastor Amorós, Jonathan Rubio García, Esther Navarro Roldán, Janina Llorens Vázquez, Spain

Email: r.sola@primhospitales.com

Aim: A comparative research project focusing on the differences noticed between major burnt patients receiving transpyloric feeding versus major burnt patients receiving usual gastric feeding. Furthermore the implementation of a new transpyloric feeding protocol in a burn unit. Methods: Review of publications by previous research teams on this conceptual bases. A calculation of individuals recommended daily calorie intake with the Harris-Benedict Principle was applied, once the patient was admitted on our inclusion criteria according to assessment through the abbreviated burn severity index (ABSI). Some nutritional markers (albumin, total proteins) were monitorized. Results: A case-control was performed, with an assessment of demographic data, therapeutic intensity and nutritional and metabolic basal data to prove an homogeneous sample. The outcomes showed an improvement of nutritional deliveries data and a better tolerance for the transpyloric group. An improvement of cicatrization of healing wounds was stated. Interruption reasons in both techniques (gastric and transpyloric) was registered and analyzed. Conclusion: Enteral transpyloric delivery of nutrition is better than gastric delivery to cover nutritional requeriments in a more effective and quick way, which impact affects cicatrization time. This procedure reduces time between surgeries and their time of recovery. References: 1) Grau Carmona T, Rincon Ferrari MD, Garcia Labajo et al. Nutritional Support in Burnt Patients. Nutr Hosp. 2005 2) Prelack K, Dylewski M, Sheridan R. Practical Guidelines for Nutritional Management of Burn Injury and Recovery. Elsevier 2006 doi:10.1016/j.burns.2006.06.014 3) Ukleja A, Sanchez Fermin M. Gastric versus Post-pyloric Feeding Relationship to Tolerance, Pheumonia Risk and Successful Delivery of Enteral Nutrition. Endocrinol Nutr 2007 Aug;9(4)309-16.

Targeting hypoglycemia in the ICU with evidence-based practice strategies

\section{Ruth Kleinpell, United States of America}

\section{Email: Ruth_M_Kleinpell@rush.edu}

Overview: Glycemic control has evolved as a standard of care in the intensive care unit (ICU). Yet, achieving glucose control with use of intensive insulin protocols is associated with hypoglycemia, a condition with the potential for adverse patient outcomes. Limited research has explored the impact of nursing care measures to impact 


\section{* European Critical Care Nursing: Working Together for a Better Tomorrow.}

2011 congress abstracts *

glycemic control for ICU patients. Aim: This study sought to: 1) Identify factors influencing episodes of hypoglycemia that impact ICU nursing care oversight of insulin protocols and 2) Examine the impact of web based education for ICU nurses knowledge and adherence to insulin protocols. Methods: A retrospective study design was used to assess factors influencing episodes of hypoglycemia in the ICU. Episodes of hypoglycemia were tracked during 3 months before and 6 months after implementation of a revised intensive insulin protocol in 4 adult ICUs at a university affiliated medical center. A web based tutorial was developed and tested to examine the impact of an educational intervention for ICU nurses on increasing awareness and adherence to established insulin protocols. Results: The number of hypoglycemic events and number of protocol deviations significantly decreased (> 50\%). Results from the post implementation surveys from 150 nurses demonstrated an increase in knowledge of protocol components compared with the pre implementation surveys from 165 nurses. Conclusions: The results of the study indicate that focused efforts with education and protocol revision can improve implementation of intensive insulin protocols, a common nurse driven practice in ICU settings. References Van den Berghe $G$ et al. N Engl J Med 2006; 354:449-461 Brunkhorst FM, et at. N Engl J Med 2008;358:125-139 The NICE-SUGAR Study InvestigatorsN Engl J Med 2009; 360:1283-1297.

Evidenced based nurse driven pain management protocol for the post operative patient

\section{Naela Hayak, Julie Benbenishty, ghislain rosen, Ety Alon, Ronit Meidan, Orly Toren, Israel}

Email: juliebenben@gmail.com

In the recovery room patients suffer from post-operative pain. Very often physicians are not available to prescribe pain alleviating medications. The patient is admitted to the recovery room while he is still slightly sedated and under the influence of anesthetic drugs given during the surgery. After evidenced based pain assesment, the recovery room nurse is left with no means in which to alleviate the patients' post operative pain. There are indications that nurse driven post operative pain protocols have enabled nurses to achieve more holistic patient assessment, resulting in more appropriate treatment (Luker \& McHugh Lewis-Evans \& Jester 2004). The purpose pain management nurse driven procotol is to (1) facilitate the safety and effectiveness of acute pain management in the perioperative setting; (2) reduce the risk of adverse outcomes; (3) maintain the patient's functional abilities, as well as physical and psychological well-being (4)enhance the quality of life for patients with acute pain during the perioperative period. (5)- shorten the recovery room patient stay to facilatate effiecincy of resources in operating theatre and recovery room. We propose to present to you the evidenced based post operative nurse driven protocol that we have developed. We preformed an initial explorative study including 100 post operative patients in the recovery room. The data collected showed high pain VAS scores with prolonged relief times while nurses watied for pain medication orders from physicians. In order to alleviate this unacceptable situation, the nursing staff of 2 campuses of a university hospital developed an evidenced based nurse driven pain protocol. We offer to present our pre protocol data, the process of nurse driven protocol development and the final evidenced based nurse driven pain management protocol for the post operative patient.

Cypriot nurses' compliance with standard precautions to avoid exposure to pathogens

Georgios Efstathiou, Evridiki Papastavrou, Vasilios Raftopoulos, Anastasios Merkouris, Cyprus

Email: george.efstathiou@cut.ac.cy

Aim: To investigate Cypriot nurses' compliance with the main principles of Standard Precautions in order to avoid exposure to pathogens during their clinical practice. Methods: A descriptive, comparative, correlational research study was employed. A 10 item, Likert type questionnaire, measuring the frequency of implementation of Standard Precautions, was administered to a convenience sample of 668 nurses from various clinical disciplines, during March-May 2010. Response rate was $89.37 \%$. Results: Full compliance (always follow) among nurses on individual aspects of Standard Precautions varied, ranging from $30.2 \%-95.7 \%$. Compliance with all the main principles of Standard Precautions was reported only by the $9.1 \%$ of the participants. Male nurses showed better behaviour than women $(p<0.001)$, and age and the frequency of implementation were positive and significantly correlated (\&\#961; $=0.185, p<0.01)$. Needlestics was the main mode of being exposed to pathogens Conclusion: The findings identified inadequate compliance with Standard Precautions on various aspects. This fact may lead nurses to occupational exposure to pathogens. Intensive care units are departments where the literature reports high incidences of exposure due to several reasons; therefore the risk of acquiring a serious disease is higher. It is vital to examine nurses' behaviour in these workplaces, in order to take appropriate measure when needed and enhance their safety within clinical practice References: 1) Gamon J., Morgan-Samuel H., Gould D. (2008) A review of the evidence for suboptimal compliance of healthcare practitioners to Standard/ Universal infection control precautions. Journal of Clinical Nursing, 17: 157-167 2) Kermode M., Jolley D., Langkham B, Thomas MS., Wendy Holmes W., Sandy M. Gifford S. (2005) Compliance with Universal/Standard Precautions among health care workers $\mathrm{n}$ rural north India. American Journal Infection Control, 33:27-33.

\section{SESSION 12: ADVANCING PRACTICE}

Making the long-term intensive care patient a positive challenge to the staff.

\section{Rikke Lundquist, Lone Jensen, Denmark}

Email: rikkelundquist@hotmail.com

Background: Studies have shown that once specific nursing guidelines have been implemented, the interest in the guidelines as well as the quality of the care of long term hospitalised patients' decreases (Hansen et al. 2008). Motivation must contribute to increased focus and enhanced quality in this area (Graversen, Holt Larsen 2004). The intensive care staff at our department has experienced that the treatment and care of long term hospitalised patients must be given greater priority. Purpose: To increase focus on treatment and care of intensive care patients admitted for more than ten days. Method: A cross-disciplinary working group was formed in September 2009. The purpose was to develop an implementation strategy for increasing quality in the treatment and care of long term admitted intensive care patients. Key staff was continuously educated in professionally relevant subjects. They were responsible for introducing colleagues to the project and support the daily clinical cross-disciplinary collaboration. The project was evaluated by a questionnaire before a workshop and six months after implementation. Results: The first questionnaire study showed that $24 \%$ of the respondents believed it to be challenging to treat and care for the long term admitted patients. However, only $8 \%$ stated that they had adequate knowledge. In October 2010 the questionnaire is sent out again and will show if the individual staff groups in the department believe there has been a change in attitudes towards this patient group. Graversen, G. \& Holt Larsen, H. 2004, Arbejdslivets psykologi, 1. udgave edn, Hans Reitzel, Kbh. Hansen, B.S., Fjaelberg, W.T., Nilsen, O.B., Lossius, H.M. \& Soreide, E. 2008, "Mechanical ventilation in the ICU--is there a gap between the time available and time used for nurseled weaning?", Scandinavian journal of trauma, resuscitation and emergency medicine, vol. 16, pp. 17. 
Intra-aortic balloon pumping and nursing care: what did we learn in the last decade?

\section{Gianluca Dilettoso, C.Anelli, D.Balestri, A.Crespi, Italy \\ Email: gianluca.dilettoso@tin.it}

Background: Now, nearly 40 years after the its introduction in the clinical practice, the intra-aortic balloon pump (IABP) is the most widely used temporary cardiac assist device. Aim: Greater awareness of nursing professionals, determined also by the advent of new technologies and medical devices, together with the higher complexity of in-patients, leads to continuous reflections on the expertise, the education and training of the nursing staff operating in the postoperative setting of Cardiothoracic ICU. Results: In the last 10 years, complications associated with the use of IABP have significantly decreased. This reduction is due to the technical improvement of materials, to the wider use of percutaneous techniques and to the more and more frequent use of IABP in patients with cardiogenic shock, factors that have contributed to the improvement of the learning curve of medical and nursing professionals. From the experience acquired in the treatment of cardiogenic shock and in the use of the IABP, it has emerged the unavoidable need to resort as soon as possible to IABP and intensive care to avoid multi-organ damages highly associated to mortality. The reduction of complications is ensured by a peculiar and scrupulous nursing practice, which critically betters itself on a constant basis. Implication for practice: The ICU nursing professional, who works with more and more sophisticated techonolgies and devices, has always to be be acquainted with current literature, in order to ensure a better nursing care and to reduce complications. References: Lewis PA, Ward DA, Courtney MD. The intra-aortic balloon pump in heart failure management: implications for nursing practice. Aust Crit Care. 2009 Aug;22(3):25-31. Tremper RS. Intra-aortic balloon pump therapy-a primer for perioperative nurses.AORN J. 2006;84(1):34-44 Elahi MM, Chetty GK, Azeem T, Complications Related Intra-aortic balloon pump in cardia surgery: a decade later. Eur J Vasc Endovasc Surg. 2005;29:591-594.

Does the infusion flow rate through the distal lumen of a central venous catheter influence the continuous monitoring of central venous pressure? A prospective pilot study

\section{Barbara Gazzini, Giovanni Cianchi, Giovanni Zagli, Stefano Bambi, Italy}

Email: murimuri2006@libero.it

Aim: Central Venous Pressure (CVP) monitoring is a widespread haemodynamic parameter in the critical care settings. Usually the CVP monitoring transducer system is connected to the distal lumen of the Central Venous Catheter together with continuous fluid infusion administered through a three way tap. The aim of this pilot study was to verify if the values of CVP monitored through a distal lumen of a CVC, were influenced by different infusion rates of normal saline administered through the same lumen. Method: A prospective observational crossover study. The sample was composed by all the adult mechanically ventilated patient admitted in Emergency ICU of Azienda Ospedaliero Universitaria Careggi from 03/01/2009 al $03 / 31 / 2009$, with a three-lumen CVC placed in superior vena cava. CVP values were randomly collected with normal saline infusion flow rate ranging from $0 \mathrm{~mL} / \mathrm{h}$ (reference standard measure) to 10,20 , $30,40,50,80 \mathrm{~mL} / \mathrm{h}$. During the values measurement of CVP, the operator was blind to the actual infusion flow rate. Results: 21 patients were included, and 7 measurements were performed in every single patient. The difference of the recorded mean values of CVP between flow rate of $0 \mathrm{~mL} / \mathrm{h}$ and $80 \mathrm{~mL} / \mathrm{h}$ was $0.9 \mathrm{mmHg}$ (repeated measure ANOVA test $P<0.0001$; linear trend post test $P<0.0001$ ). No statistical differences were found when CVP values were adjusted for Positive End Expiratory Pressure values. Conclusions: CVP measures are significantly influenced by the tested infusion rates, but not clinically relevant for diagnostic or treatment decision. References Ho AM et al. "Accuracy of central venous pressure monitoring during simultaneous continuous infusion through the same catheter". Anaesthesia, 2005; 60:1027-1030 Lakhal K, et al. "Influence of infusion flow rates on central venous pressure measurements through multi-lumen central venous catheters in intensive care". Intensive Care Med. 2006;32(3):460-3. Epub 2006 Feb 14.

A randomized controlled trial to determine the effectiveness of tea tree oil body wash in preventing MRSA in critically ill patients

Gillian Thompson, Bronagh Blackwood, Ronan McMullan, Fiona Alderdice, John Trinder, Danny McAuley, United Kingdom

Email: gillian.thompson@belfasttrust.hscni.net

Aim: To determine effectiveness of $5 \%$ tea tree oil body wash in preventing colonisation with methicillin resistant Staphylococcus aureus (MRSA) in critically ill adults. To achieve this, we undertook a systematic review of the literature and found that tea tree oil was effective in killing MRSA in the laboratory setting1, and it was a promising intervention to treating MRSA colonisation in the clinical setting2,3. However, there was no evidence of its effectiveness in preventing MRSA colonization. Methods: To investigate this further, we undertook a prospective, randomized controlled trial. The trial was undertaken in a large intensive care unit in the UK. Patients were assigned to one of two groups, to be washed daily with one of the following interventions: $5 \%$ TTO body wash or standard care body wash for the duration of their stay in Intensive Care. Nasal and groin swabs were taken on admission and discharge from the unit and the outcome measure was MRSA colonization. Results: There were 391 patients that completed the trail. The trial was terminated early due to low accrual and therefore the study was underpowered to detect a statistical difference. The results showed a $2.5 \%$ difference in colonization rates in favour of TTO but the difference was not statistically significant $(95 \% \mathrm{Cl} 8.95$ to $3.94, \mathrm{p}=0.50)$ nor clinically significant. Conclusion: Although tea tree oil cannot be recommended for preventing MRSA colonization, this study adds to the body of nursing research in many ways. First, it is a useful pilot study that provides important insights for further research in this area. Second, it highlights that tea tree oil is safe to use and well tolerated. References 1. Carson et al. J Antimicrob Chemother 35: 421-4 2. Caelli et al. J Hosp Infect 46: 236-7 3. Dryden et al. J Hosp Infect 56: 283-6.

\section{Can aromatherapy reduce anxiety in intensive care patients}

\section{Gitte Frandsen, Damon Grech, Denmark}

Email: gitte_frandsen@msn.com

Background: Admission to an intensive care unit is often associated with anxiety. Intensive care staff attempt multiple strategies to minimize discomfort and stress for the patients. At our unit we have not previously worked consciously with the association between aromatherapy and anxiety. International research has shown that the smell of lavender can reduce anxiety (Itai et al. 2000), though not in cuffed patients. Aim: To investigate if lavender aromatherapy can reduce anxiety and discomfort in intensive care patients. Material and Method: The study period will be five weeks. We use aromatherapy for three weeks during week 2-4. Essential lavender oil will be spread in the air by an aroma stream evaporator twice a day for two hours. . Between three and five patients, cuffed or un-cuffed, will be enrolled in the study. The patients will be scored by the Pittsburgh Agitation Scale (PAS-score) (Zieber et al. 2005) three times a day. The experiences of the staff will also affect the result of the study; the nurses thus evaluate their personal experiences, changes in patients and experiences of relatives three times a day. Results: The study will show if the PAS score of patients is reduced. Moreover, if the use 
of aromatherapy has positively influenced the patients' surroundings. References: Itai, T., Amayasu, H., Kuribayashi, M., Kawamura, N., Okada, M., Momose, A., Tateyama, T., Narumi, K., Uematsu, W. \& Kaneko, S. 2000, "Psychological effects of aromatherapy on chronic hemodialysis patients", Psychiatry and clinical neurosciences, vol. 54, no. 4, pp. 393-397. Zieber, C.G., Hagen, B., Armstrong-Esther, C. \& Aho, M. 2005, "Pain and agitation in long-term care residents with dementia: use of the Pittsburgh Agitation Scale", International journal of palliative nursing, vol. 11 , no. 2 , pp. 71-78.

\section{SESSION 13: PAIN MANAGEMENT}

Substance P and NPY plasma levels in adults with chest pain due to coronary artery disease:Potential indicators for nursing assessment

Eleni Kletsiou, Elizabeth D.E Papathanassoglou, Evangelos Bozas, Efstathios K. Iliodromitis, Maria Anastasiou-Nana, Margarita Giannakopoulou, Greece

Email: eklets@nurs.uoa.gr

Aim: Substance P (SP) and neuropeptide Y (NPY) are neuropeptides involved in nociception. Purpose of the study was to explore potential associations between plasma neuropeptide levels and reported pain intensity in coronary critical care adults, in order to test the reliability of SP measurements for objective pain assessment in critical care. Method: A descriptive correlation repeated measures design was employed. A group of 25 coronary critical care adults with retrosternal pain, fulfilling the criteria of acute coronary syndrome, and a control group of 25 healthy gender-age matched volunteers were studied. Blood samples were collected during an episode of pain associated with ECG changes and 24 hours later, where no pain or ECG changes were reported. Neuropeptide levels were quantified by an immunosorbent (ELISA) assay. The level of pain was assessed by behavioural pain scales (Payen's 2001- Puntillo's 1997 pain scale) and the numeric analogue scale. Clinical severity was quantified by APACHE-II and the Multiple Organ Dysfunction Score (MODS). Results: NPY and SP levels were significantly increased in patients with coronary artery disease during the episode of pain versus no pain ( $p=0.03,0.04)$ respectively. In patients' group, a positive correlation between SP and NPY levels respectively and VAS pain ratings $(r=0.474,0.565, p=0.02)$, ratings at the Puntillo $(r=0.474$, $0.563, p=0,010)$ and the Payen scales $(r=0.562,0.737, p<0.0001)$ was observed. SP and NPY levels exhibited significant inverse association with MODS scores $(r=-0.404-0.450, p<0.03)$. Significant differences between patients with acute myocardial infarction and controls were found in SP (Äl $=-199.97 \mathrm{pg} / \mathrm{ml}, \mathrm{p}=0.04)$ while significant differences in NPY levels were observed in all patients with coronary artery disease compared to the controls $(p<0.04)$ Conclusions:The observation of elevated SP and NPY plasma levels in coronary critical care patients and of associations with subjective pain perception and pain scale scores may have important clinical implications.

Pain assessment in adult ICU: Critical-Care Pain Observation Tool or the Behavioral Pain Scale?

\section{Willemke Stilma, CAM Koper, S Rijkenberg, The Netherlands}

Email: w.stilma@olvg.nl

Background: Among ICU patients, the presence of pain may influence the recovery and the occurrence of complications. Various pain scales are available to assess pain in ventilated patients, such as the Critical-Care Pain Observation Tool (CPOT) and the Behavioral Pain Scale (BPS). Aim: To test the CPOT and BPS scales by nurses on inter-rater reliability and the expressiveness for pain in different situations. Method: Prospective observational study in a 26-bed ICU. Adult ventilated patients were randomized in a Cardio-thoracic surgery group and a group with other disciplines. Analysis was directed to assess inter-rater reliability and discriminant validity of the BPS and CPOT. BPS and CPOT were scored by two nurses during patient's rest, teeth care, and turning of the patient because the scores might give an indication of the way the scales are discriminative to pain. Data collection was between May and September 2010. Results: Totally, 497 patients met the inclusion criteria in the study period. Of these, $332(67 \%)$ were observered. Preliminary results showed that of observed patients 154 patients $(46 \%)$ could be included in the analysis. This difference occurs because not all 4 measure moments could be performed due to extubation or other reasons. Analysis of the data is in progress and are expected to be finished in November 2010. Final results will be presented at the EfCCNa congress in March 2011. Conclusion: The study results will guide the decision to choose for the best reliable scale to use in clinical practice, including its feasibility. References: 1. Li D, Puntillo K, Miaskowski C. A review of Objective pain measures for use with critical care adult patients unable to self-report. J Pain 2008;9:2-10. 2. Puntillo K.A, Morris R.N, Thompson C.L, et al. Pain behaviors observed during six common procedures: Results from Thunder Project II. Crit Care Med 2004;32:421-7.

Further assessment of a behavioral pain scale or Dolousi Pain Scale in patients who are unable to communicate

\section{Marie De Val, M Pachioli, C Lorent, T Nguyen, Belgium \\ Email: marie.deval@skynet.be}

Aim: In a previous communication, we proposed a behavioral scale developed for assessing pain in Non Communicating Patients (NCP) the Dolousi Pain Scale (DPS). The aim of the present communication is to evaluate the specificity, the inter-rater agreement and the internal consistency of this scale. Methods: 110 critically ill patients unable to express their pain either verbally or by clear signs were evaluated according to the DPS. The 4 behavioral items were recorded during 3 different situations: at rest, during a slightly painful procedure and during a more painful procedure. This scale evaluates four items: adaptation to ventilation, face expression, motricity and tears production (minimal score $=4$; maximal score : 15 ). During the study period, all patients were intubated or ventilated with analgosedation or sedation. GLASGOW score was inferior or equal to 10 and RAMSAY score superior or equal to 4 (sedation). Statistical analysis demonstrated significant differences between the 3 situations ( $P<0,001$ Wilcoxon test). To answer the question "Does our scale specifically assess pain?" we performed a further prospective evaluation in 45 NCP using a pressure on the nail of the thumb during 5 secondes. Results: Using the DPS, this unquestionable painful stimulus yielded very significant differences as compared to rest. ( $P<0,001$ Wilcoxon test) Furthermore the inter-rater agreement between two independent examiners, evaluated by the Spearman Rank order correlation, revealed an excellent inter-rater agreement, as applied to the same cohort of patients $(0,95$ to 0,90$)$ At last, the Cronbach á index showed a very good internal consistency $(0,74$ to 0,73 ). Conclusions: The DPS is a valid tool for measuring pain in NCP. This scale yields reproducible findings across examiners and has a very good internal consistency.

\section{Memories of pain after burn injury: the patient's experience}

\section{Oili Dahl, Marie Wickman, Yvonne Wengström, Sweden}

Email: oili.dahl@karolinska.se

Aim: Pain after burns is major clinical problem and researchers continue to report that burn pain remains under-treated. The purpose of this study was to describe burn patient's experiences and memories of pain during burn care and to acquire a deeper understanding how patients cope with the pain. Methods: The study 


\section{* European Critical Care Nursing: Working Together for a Better Tomorrow.}

2011 congress abstracts *

method was qualitative and interviews were conducted with 12 burn patients, 6-12 month post burn. Purposive sampling was used and the inclusion criteria were $>18$ year, no ventilator-treated patients, length of stay $>7$ days and cognitive and communicative abilities. The interviews were analysed using Kvales method for structuring analysis. Results: All the patients had the factual memories of the pain and the care. The patients' experiences and memories of pain during the trajectory of care were clearly described in chronological order by the informants. Four themes were identified for pain this including sub themes; becoming aware of pain, allowing oneself to feel pain, different pain experiences and fragile body surface. Four themes were find for coping including one sub theme; pragmatic coping, allowing someone to care for you, carrying the pain and perspectives. Conclusion: It is evident that the patient has to carry the pain experience and the situation to a large extent by themselves. The results indicate that there need to be an increased focus on pain management and psychosocial support from the experienced staff to the patients in the future to enhance coping and return to normal life

\section{SESSION 14: EDUCATION AND COMPETENCY}

Undergraduate nursign students and critical care: How an innediate life support course can help improve skills and critical decision making

Billiejoan Rice, Patrick Gallagher, Niall Mc Kenna, Dr Marain Traynor, Trevor Mc Nulty, United Kingdom

Email: b.j.rice@qub.ac.uk

This paper outlines how the immediate life support (ILS) course was incorporated into an undergraduate-nursing curriculum in a university in Northern Ireland. It also reports on how the students perceived the impact of this course on their clinical practice. The aim was to develop the student's ability to recognise the acutely ill patient and to determine the relevance of this to clinical practice. Prior to this the ILS course was only available to qualified nurses and this paper reports on the first time students were provided with an ILS course in an undergraduate setting. Methods The ILS course was delivered to 89 third year nursing students (Adult Branch) and comprised one full teaching day per week over two weeks. Recognised Advanced Life Support (ALS) instructors, in keeping with the United Kingdom Resuscitation Council guidelines, taught the students. Participants completed a 17 item questionnaire which comprised an open-ended section for student comment. Questionnaire data was analysed descriptively using SSPSS version 15.0. Open-ended responses from the questionnaire data was analysed by content and thematic analysis. Results Student feedback reported that the ILS course helped them understand what constituted the acutely ill patient and the role of the nurse in managing a deteriorating situation. Students also reported that they valued the experience as highlighting gaps in their knowledge Conclusion. The inclusion of the ILS course provides students with necessary skills to assess and manage the deteriorating patient. In addition the data from this study suggest the ILS course should be delivered in an inter-professional setting - i.e taught jointly with medical students. References: Resuscitation Council (UK) (2006) Immediate Life Support. (2nd edition) United Kingdom: Resuscitation Council (UK) Soar, J., Perkins, G, D., Harris, S. \& Nolan, J. (2003) The immediate life support course. Resuscitation; 57: 21-26.
Competencies for postgraduate critical care nurses: developing a tool for the evaluation of nurses' perceptions

Maria Hadjibalassi, Evridiki Papastavrou, Ekaterini Lambrinou, Evanthia Georgiou, Evdokia Athini, Elisavet Nicolaou, Anastasios Merkouris, Cyprus

Email: maria.hadjibalassi@cut.ac.cy

Aim: To develop an instrument concerning nurses' perceptions of postgraduate level competencies for critical care nurses. Methods: A three-phase approach was followed. a) Content development and critique, b) pilot study and c) field study. In the first phase focus groups were used to collect information from the nurses involved in clinical practice. Data gathered from focus groups formed the basis for the development of a tool which then was discussed among the research team and the underlying concepts of each item was evaluated in terms of clarity, intelligibility and appropriateness to the target population. Then, resulting competencies were discussed within a group of 24 critical care nurses. Feedback was sought from 15 clinicians and academics prior to finalization and pilot testing of the tool. In the last phase 234 questionnaires were distributed among critical care nurses in Cyprus with a response rate $66 \%$. Psychometric approaches such as internal consistency reliability using Cronbach's alpha and construct validity were used to validate the instrument. Exploratory factor analysis was used to determine the factor structure of the instrument. Results: Four factors were revealed out of the finalized questionnaire, which included 72 items: 1. Leadership/Management and Professional Development, 2. Decision making and management of emergencies, 3. Care Delivery and 4 . Ethical sensitivity. All factors were highly reliable, with Cronbach's alpha ranging from 0.895 to 0.974 . Conclusion: A Likert scale questionnaire was generated that reflects the multidimensional and holistic character of the critical care nurses' role. Apart from the statistical support, these factors may well maintain the International Council of Nursing (ICN) Framework of competencies.

Technical, interpersonal and critical thinking competency assessment: a systemic approach

\section{Sabrina Egman, Santa Giammona, Italy}

Email: segman@ismett.edu

Competence is more than possessing the knowledge or psychomotor skills necessary to perform a specific task. For professional nurses, competence means that the caregiver integrates knowledge, skills and personal attributes consistently in daily practice to meet established standards of performance. The significant growth in key clinical hospital services requires educators to examine nursing competency to evaluate areas that need reinforcement in order to guarantee the high quality of care required by the $\mathrm{JCl}$ standards. This session presents our current Institution standards to assess nursing competencies needed to measure critical thinking and clinical decision-making abilities and necessary as a result of findings from quality- improvement data. To assess nursing competency a methodological plan had to be established. Competencies have two major components: the first is a competency statement describing the general performance standard; the second is a list of criteria describing the tasks required to ensure that the general performance standard is met. Before beginning the competency development process, four important aspects had to be considered: outcome, category, learning domain and audience. Expected outcomes were clearly defined based on pre-established goals with the aim of assessing acquisition of a new skill or of validating existing knowledge and skills. Competencies were selected as generic for evaluating the skills and knowledge needed to execute fewer complexes nursing interventions and intermediate or advanced in order to evaluate those needed for more complex interventions. Identifying a particular learning domain was needed to address competencies. Novice nurses 


\section{* European Critical Care Nursing: Working Together for a Better Tomorrow.}

2011 congress abstracts *

needed a high percentage of psychomotor competencies, whereas competent nurses needed validation of higher level competencies. Clear identification of the target audience promoted understanding of the intent of the competencies. Competency assessment has been proved as a valid tool for building long-term continuous improvement of high quality nursing care.

Major components of nurses: evaluation of nursing diagnosis according to NANDA and nursing care in the internal medicine ICU

\section{Sevgin Samancioglu, Deniz Unlu, Fehmi Akcicek, Turkey \\ Email: sevginsamancioglu@hotmail.com}

Aim: Nursing practice is a major component of health care. Nursing diagnosis and activities classification systems are necessary for representing important aspects of "what nurses do". So, aim of this study identify the prevalent Nursing Diagnoses (ND) in a Intensive Care Unit (ICU); to compare the prevalent ND with the recorded cares for their respective diagnoses. Methods: Data was obtained in a university hospital Internal Medicine ICU in the period of three years (2007-2009), and the study, which was carried out retrospective, based on such data indicated that interventions differed from one patient to the other, pointing out that nursing care was individualized to daily patient need and was continued right up to the time of hospital discharge. Results: In one analysis of nursing diagnosis - used mostly during an ICU stay and documented in a database - the patients were divided into two groups: Nephrology patients $(n=242)$ and Hematology patients $(n=179)$. Each patient in the internal medicine icu received an average of 16 different interventions. Conclusion: In this group, $53.4 \%$ were men, $46.6 \%$ were woman, the mean age was 61.8 with a median length of stay of 6 days. Nine interventions were used in a high rate and a high percentage of hospitalization: intravenous therapy(96.2\%), blood culture $(87.3 \%)$, uriner catater $(81.4 \%)$, hyperthermia(78.9\%), oxygen therapy $(76.3 \%)$, pain management $(59.3 \%)$, position $(57.2 \%)$, nausea $(44.6 \%)$, hypothermia $(18.9 \%)$. Six prevalent ND of the 62 ND prescribed are: Fluid Volume Deficit/Fluid Volume Excess (58.1), Ineffective Breathing Patterns(43.9), Risk for Infection $34.2 \%$, Imbalanced Nutrition(25.2\%), Altered Thought Processes, (24.1\%), Uriner Incntinanse(13.1\%). Six prevalent ND were identified with the recorded nursing care. However, other care could have been established as a priority. Reference:Maria Müller-Staub. Evaluation of the Implementation of Nursing Diagnoses, Interventions, and Outcomes. International Journal of Nursing Terminologies and Classifications Volume 20, No. 1, 2009.

\section{SESSION 15: WORKSHOP}

\section{Workshop: Nursing activities score-share our experiences}

Siv Stafseth, Norway, Katia Padilha, Brazil, Javier Carmona, Spain, Marga Hoogendoorn, The Netherlands

Background and Aim of the workshop: The tool Nursing Activities Score (NAS) was developed to measure patient care by Miranda et al. in 2003. Research on the topic was performed in Spain and Brazil, for example studies from Adell et al, 2005 and Padhila et al, 2010. NAS is in use to estimate the nurse staffing in patient care for the ICUs. The aim of the workshop is to share experiences in international use of NAS to different groups of patients, to discuss the items in NAS and get a consensus of the score. Finally to discuss an upcoming multicentre research project that includes; patient safety, nursing incidents/adverse events and association with nursing workload, professional burnout, nursing satisfaction and safety environment. Method of presentation: Lectures in experiences from Norway, Brazil, Spain, the Netherlands and a discussion on the topic.
Learning Outcomes: To introduce participants to evidence-basedresearch studies on the area of nursing workload. References: Adell AB, Campos RA, Cubedo RM, Quintana BJ, Sanahuja RE, Sanchis MJ, et al. Nursing Activity Score (NAS). Our experience with a nursing load calculation system based on times. (Article in Spanish) Enferm Intensiva 2005;16(4):164-73. Miranda DR, Raoul N, Rijk A, Schaufeli W, lapichino G. Nursing activities score(NAS). Critical Care Medicine 2003;31(2):374-82. Padilha KG, Sousa RMC, Garcia PC, Bento ST, Finardi EM, Hatarashi RHK. Nursing workload and staff allocation in an intensive care unit: A pilot study according to Nursing Activities Score (NAS). Intensive Critical Care Nursing 2010;26:108-13.

\section{SESSION 16: CROSS CULTURAL CARE}

Challenges of haemorrhagic fever outbreaks: reflect on the past and look to the future

\section{Andrea Hayward, Jean Erasmus, South Africa}

Email: hayward.ar@gmail.com

BACKGROUND: Prevention of the spread of Viral Haemorrhagic Fevers(VHF) in the developing world comes with many challenges. Many viruses that are known to cause disease in humans are found in Sub-Saharan Africa (Old World Virus) and South America (New World Virus) Very few occur naturally in South Africa. However travel between North and South Africa has increased the possibility of importing VHF significantly. Over the last five years a number of patients presented with a variety of types of VHF. The latest outbreak was Rift Valley Fever in 2010 and included 25 cases. A significant event that made the international news was the novel arenavirus called Lujo, and is the first highly pathogenic arenavirus to be identified in Africa in 50 years. These outbreaks can be considered as Medical Disasters and are costly to both the country as well as the institution to which they are admitted. RESULTS:At a single private hospital, we have admitted 4 cases, 3 with Lujo arenavirus and 1 with Dengue fever in the last 2 years. 5 patients involved in the Lujo virus outbreak. 4 of these died before a diagnosis was confirmed. Standard precautions used initially were stepped up to water repellant bodysuits. Accurate timelines were established. Protocols for tracing and monitoring contacts were implemented. CONCLUSIONS:The skills obtained during these outbreaks and the post outbreak analyses have given those involved an enormous amount of information on managing this type of patient. Guidelines need to be in place for admission of patients from endemic areas. IMPLICATIONS FOR PRACTICE:With international travel, VHF is easily imported. Port Health regulations need to be strictly applied in transfer of patients. Appropriate infection control precautions and constant revision of guidelines are required. Particular attention to removal and disposal of protective clothing must be enforced.

Emergency, triage and teaching in Zambia: working exeprience

\section{Drago Satosek, Slovenia}

Email: drago.satosek@kclj.si

Background: The Slovenian Society for Tropical Medicine provides programs to the medical and nursing students, nurses, and doctors who are enthusiastic to work in developing countries. The programs aim to bring free medications, equipments, and experienced staff to provide nursing care, to teach local nurses, and to help people in tropical rural areas. Aim: To share knowledge of working in an emergency hospital setting in a developing country and to discuss the cross-cultural implications for critical care nursing. Results: The Missionary hospital Nangoma in Zambia has 30 adult beds and 20 pediatric beds as well as 20 beds for post surgery patients and post delivery mothers. There is one physician for 24 hours and 3 nurses 


\section{* European Critical Care Nursing: Working Together for a Better Tomorrow.}

2011 congress abstracts *

per shift to look after all hospitalized patients. The hospital also provides emergency care. Most of the emergency patients have high fever, unconscious patients due to malaria or neuropathy connected to AIDS, injuries, acute abdomen, or severe burns. The patients are transported to the hospital by bicycle, on a carriage (by ox and rarely by horse), and very rarely by car. All critically ill patients are admitted to the ward directly and then triage is done to prioritize nursing and medical care. It is important to collect all data to make the right decision. Implication for Practice: As we are guests in the Missionary hospital, it is not advisable to impose our 'western principles'. It is important to give good example by providing quality care and doing good triage. Discussing real-time case studies to improve clinical practice based on the available resources, the local staff realizes the importance of triage and emergency care. Over time the local nursing staff tries to change their practice towards good clinical practice to improve patient's outcomes.

Building medicine in Africa: The Salam Centre for cardiac surgery in Khartoum, Sudan

\section{Chiara Peduto, Italy}

\section{Email: chiarapeduto@gmail.com}

BACKGROUND: The epidemiology of cardiovascular diseases in Africa differs across certain groups of the population, probably due to different exposure to known and unknown risk factors, unhealthy lifestyles, access to health care, and environmental conditions, including traditional customs. Diseases linked to poverty and famine are still highly prevalent. Health infrastructures adequately equipped for diagnosis and care are lacking, and there is an absence of research to deliver the required scientific advances in a reasonable timescale. The Salam Centre for Cardiac Surgery is run and managed by the Italian based humanitarian organisation EMERGENCY. Since April 2007, the Centre provides high standard, free of charge paediatric and adult cardiac surgery. AIM: to offer high standard surgical care to children and adults suffering from acquired or congenital heart diseases RESULTS: From April 2007 to August 2010: Patients triaged 25,552; Cardiological examinations 17,177; Number of hospital admissions 4,031; Number of patients admitted 3,243; Number of surgical operations 2,628; Number of patients operated 2,526; Cath Lab procedures 841 . Patients' distribution by type of disease: Valvular Heart Diseases $70.2 \%$; Congenital Heart Diseases 23.6\%; Ischemic Heart Diseases 4.8\%; Disease of the Aortic Valve and Ascending Aorta 0.7\%; Ascending Aorta 0.1\%; Disease of the Aortic Valve and Ascending Aorta and CABG 0.04\%; Other 0.5. The surgical mortality (30days mortality) is $3.13 \%$. IMPLICATION FOR PRACTICE: In accordance with The Universal Declaration of Human Rights, EMERGENCY recognizes the right to be cured as a basic and inalienable human right. It works to implement Human Rights based Health Projects, according to the principles of equality, quality and social responsibility. The Salam Centre promotes cooperation and solidarity between different peoples and cultures. On the other hand the Centre promotes a high quality local staff training. REFERENCES: www.salamcentre.emergency.it ; Neglected Cardiovascular Diseases in Africa: Challenges and Opportunities - A.O.H. Mocumbi, M.B.Ferreira J. Am. Coll. Cardiol. 2010;55;680687.

\section{SESSION 17: PATIENTS' AND NURSES' PERCEPTIONS}

The visiting situation in an ICU: An observational hermeneutic study

\section{Thomas Eriksson, Ingegerd Bergbom, Berit Lindahl, Sweden}

Email: thomas.eriksson@vgregion.se

Aim: The aim was to interpret the interplay between critically ill patients and their next of kin in an ICU to understand the visiting situation. Method: A hermeneutic research design with nonparticipant observation was chosen as the data collection method. Ten observations of 10 patients and 24 loved ones over a 20 hour period were conducted. The text describing the observations of the interplay was interpreted in accordance with Gadamer. Data were analysed by considering the text as a play with scenes, actors and plots. Results: The interpretation of the scenes revealed two themes describing the patient's interplay and six themes describing the next of kin's interplay. The understanding of the plot concerns people who had entered a situation where normal everyday life was disconnected. The path from health to illness was a totally unfamiliar environment and perception of life, where the body constituted a new and unknown world. As a result of the fact that the patients were unable to use their bodies in the usual way, which sends different signals to their loved ones, who in turn have difficulty deciding how to respond. Both parties become trapped or locked out by their own bodies. Conclusion: A hindrance to the interplay could be the room, which was designed for medical and technical use and thus did not promote healing. The professionals were crucial for interpreting the signals from both patients and next of kin, as well as for finding caring strategies, such as physical contact, that promote interplay, which in turn create a caring and healing atmosphere.

Liberating restricted visiting policy in Greek ICUs: is it that complicated?

Archonto Athanasiou, Papathanasoglou Elisabeth, Giannakopoulou Margaret, Patiraki Elisabeth, Lemonidou Xrisoula, Greece

Email: arxountia@yahoo.gr

Aim: To explore ICU nurses' beliefs and attitudes regarding current and open visiting policies in Greek Intensive Care Units (ICUs). Method: Descriptive correlational design. Data were collected from 6 randomly selected hospitals in Athens $(n=143)$, via an anonymous questionnaire consisting of two scales to assess nurses' beliefs, a scale for the assessment of attitudes towards visitation (Marco et al 2006, Berti et al 2007), demographic questions and five open-ended questions about current visiting policy. Results: Cronbach's alpha coefficients were $0.72,0.82,0.83$ (3 scales respectively). Nurses believed that open visiting policy increases family's satisfaction $(84,6 \%)$, and provides emotional support to the patient $(89,5 \%)$, but the overall effects of visiting depend both on patients and families $(91,6 \%)$. Furthermore, it may hamper planning of adequate nursing care $(75,5 \%)$, while it may increase nurses' physical and psychological burden (87,5\%). Nurses' attitudes towards liberal visiting hours were rather negative. Nurses did not wish for open visitation to be instituted $(94,4 \%)$. There was a positive correlation between scores of beliefs and attitudes regarding visitation $(r=0.42$, $p<0.001, r=0.45, p<0.001)$. Length of work experience, staffing level and number of night-shifts/ 15 days associated independently with and predicted scores of attitudes/ beliefs toward visitation. Conclusions: There is a need to support ICU nurses in order to overcome any potential burden imposed by implementation of flexible/ open visiting policies. An improvement of nurse/ patient ratios will alleviate nurses' workload and the need for repetitive night-shifts and may affect nurses' defensive attitude towards liberal visitation. References: 1.Marco et al (2006). Intensive care nurses' beliefs and attitudes towards the effect of open visiting on patients, family and nurses. Nurs Crit Care; 11:33-41 2.Berti et al (2007). Beliefs and attitudes of intensive care nurses toward visits and open visiting policy. Intensive Care Med; 33:1060-1065. 
Nursing care plan in a full-face composite tissue allotransplantation

\section{Jonathan Rubio García, David Carbajales Álvarez, Monica Soler Rovira, Teresa Pastor Amorós, Janina Llorens Vázquez, Esther Navarro Roldán, Spain}

Email: dermatoide@yahoo.es

Aim: Facial composite tissue allotransplantation became a reality in 2005, but remains experimental surgery. Patients requier intensive nursing but few protocols exist. We reviewed current knowledge and our own experience to provide recommendations for sound nursing protocols. Methods: A literature search of human facial allotransplantation, including published data in peer review journals and press releases was performed, focusing on NANDA's nursing diagnosis standards. Furthermore our current experience on full-face allotransplantation was also reviewed. Results: Patient selection is very important since his compliance with the immunosuppressive and postoperative regime is essential. Severe complications may occur; therefore functional and aesthetic improvement must justify lifelong immunosuppression. A specialised nursing care plan for this kind of surgery had to be performed and standardised, which included intensive care protocols, pre, intra and postoperative care, psychological support and immunosuppressive drug administration. Conclusion: The pioneering team that performed the full-face transplantation in our hospital offered the patient an improved aesthetic, functional and social outcome that could not be obtained with traditional techniques. The input of nursing staff is essential for a successful outcome. This innovative procedure provides hospitals with a scientific and clinical-practice based action protocol, which includes a nursing care plan based on NANDA's diagnosis resources and recommendations. References: 1) Siemionow M, Ozmen, Selahattin, Demir, Yabuz. Prospects for facial allograft transplantation in humans. Plast Reconstr Surg 2004; 113:1425/ 2) Clarke A, Butler PEM. Facial transplantation:adding to the reconstructive options after severe facial injury and disease. Expert Opin Biol Ther 2005;5(12):1/ 3) Wiggins O, Barker J. Response to: Thorburn et al. Patient selection for facial transplantation III: Ethical considerations. Int J Surg 2004;2:119.

Attitudes and perceptions of Italian health personnel regarding the use of ICU diaries

\section{Enrico De Luca, Lucia Portis, Maddalena Galizio, Massimo} Michele Greco, Italy

Email: biloba_1@hotmail.com

ICU diaries practice appears not been widely realized in the Italian Critical Care facilities. The research objective was to gather qualitative data regarding the attitudes of Italian Intensive Care Unit (ICU) staff with respect to the eventual use and integration of ICU Diaries in daily care provision. Methods Six Italian ICUs were involved using Focus Group methodology (a group interview conducted by two researchers using open questions). Analysis of the transcription of the recorded interviews identified the salient points, based on relevance, frequency and intensity. The participants were mainly nurses with a smaller number of ICU anesthesiologist and some auxiliary nurses. The Focus Groups were held from November 2009 until April 2010. Results The following emerged: the perception of appropriateness and congruency in offering the Patient Diaries as one of the services of an IC Unit; the possibility of realizing this in each context; the perception of utility of such with respect to both patients and parents; the positive aspects for personnel; the importance of a narrative representation of one's own work. Conclusion There appears to be a certain attitude of change, a rethinking of organizational models and thus a willingness to learn and to transform practice in order to humanize care. ICU Diary practice has been perceived as a way to witness the complexity and daily routine of care provision, of the humane dimensions of care. Diary writing can become also for health personnel as a form of re-thinking their actual work. References COMBE D. The Use of Patient diaries in an intensive care unit. Nursing in Critical Care 2005; 10: 31-34 EGEROD I., et Al., The extent and application of patient diaries in Danish ICUs in 2006. Nursing in Critical Care 2007; 12:150-167 GRECO M.M., L'utilizzo dei "Diari del Paziente" in Terapia Intensiva, Scenario, 2009; 26: 22-27.

Transcatheter aortic valve implantation (TAVI) treated patients what kind of specialised nursing do they need?

\section{Susanne Nielsen, KH Lisby, Denmark}

Email: susanne.00.nielsen@rh.regionh.dk

Background: Rising age results in increasing incidence of aortic stenosis (AS) due to the calcification of the aortic valve. Approximately $2 \%$ over the age of 75 , and $4 \%$ over the age of 85 develop AS. This effects their quality of life. Until 2002 valve surgery was the only option of treatment. For the group of elderly $>80$ years this option was too high a risk, especially if they have comorbidities. Since 2002 Transcatheter Aortic Valve Implantation became an option for this the group of elderly. At Rigshospitalet Denmark, this intervention started in November 2007 and until now 50 patients have been treated. Specialised nursing for this group of patients remains undescribed, however it is necessary to investigate which kind of knowledge is needed to develop a high standard and evidencebased nursing within this group. Method: To identify the need of nursing an audit of the nursing chart were chosen. Findings from cardiac intensive care unit (CICU) and cardiac ward made it possible to investigate the experiences of the patients. An interview guide with incorporated scales was used 3 times during hospitalisation, at the admission, before discharging $\mathrm{CICU}$ and the 5th day at the cardiac ward. Furthermore "Observationcharts" were elaborated and used by nurses each day at the ward and each shift at the intensive care unit. Approximately 50 patients will be included. Results: Results from 50 patients at $\mathrm{CICU}$ will be presented. Identified nursing themes concerning dyspnea, arrythmias, bleeding, nausea, pain, confusion, fatigue, disturbance in sleeping pattern, inactivity, nervousness and anxiety was the focus of the investigation for this group of patients and their need of nursing. Conclusions: The nursing project and the consequences so far.

\section{SESSION 18: ORGANIZATION AND AUTONOMY}

\section{Critical information needs of Greek and Finnish charge nurses}

Heljä Lundgren-Laine, Heljä Lundgrén-Laine, Maria Kalafati, Sanna Salanterä, Finland

Email: hklula@utu.fi

Aim The aim of our study is to investigate what information needs intensive care units (ICUs) charge nurses have when they organize and run daily activities at the ICUs and which information is absolutely necessary for immediately made decisions concerning the ICU care management. Our study was carried out in Finland $(n=$ $515)$ between Sep-Nov 2009 and in Greece $(n=50)$ between AprJun 2010. Methods We use an online questionnaire (Webropol®). Our questionnaire based on the previous observation study and including 122 statements about information needs related to the ICU care activities. A rating scale $0-10$, from completely unnecessary to absolutely necessary, was used for each of the statements. Results In Finland, altogether 17 ICUs participated in our study and the response rate of charge nurses was $50.1 \%(n=258)$. In Greece, we recruited charge nurses from $26 \mathrm{ICUs}$ and got altogether 50 responses. The mean age of the Finnish charge nurses was 42.6 years (SD 8.6) and they had 14.9 (mean) years experience in 


\section{* European Critical Care Nursing: Working Together for a Better Tomorrow.}

2011 congress abstracts *

intensive care (with 7.9 SD). 38 of the Finnish charge nurses were men and 220 women. Finnish charge nurses assessed altogether 28 information needs to be absolutely necessary for their decision making. Most of their information needs concerned staff and material resource allocation. Our analysis with the Greek data is in progress and will be completed this year. Conclusion ICU charge nurses have several critical information needs which are crucial for the fluent flow of the activities at the ICUs. With an international sampling we are able to compare information needs of ICU charge nurses in two different countries. References Lundgrén-Laine H, Salanterä S. Think-Aloud Technique and Protocol Analysis in Clinical DecisionMaking Research. Qual Health Res 2010; 20; 565.

The ICU patient room as a healing environment: a research program based on evidence-based design

Berit Lindahl, Isabell Fridh, Lotta Johansson, Sepideh Olausson, Susanne Knutsson, Ingegerd Bergbom, Sweden

Email: berit.lindahl@hb.se

Aim: This research project aims at investigating if a specially designed patient room in an intensive care unit (ICU) affects people who stay there i.e., patients, next-of kin and staff compared to an ordinary ICU patient room (control). Methods: The presentation is an example of research that implements evidence-based design in an ICU. The project has a naturalistic, explorative and descriptive design. Refurbishment of a patient room took place during spring 2010. It was based on a careful co-operation between scholars, ICU care practitioners, architects, designers and experts of health care technology. A systematic review of literature describing physical environment in hospitals was also performed. The intervention room was refurbished according to research findings (Ulrich, 2006) regards to sound, light, shape and access to nature. New and innovative products, e.g. lightning, technical equipment were used. Results: The project started with a pilot study measuring sound levels in patients' rooms (Johansson et al. 2010). Then a two-bed room at the ICU was refurbished. This room was planned according to holistic principles comprising position of technology, furniture, textiles, light and sound environment. An identical room (control) remained in the original state. The presentation is a contribution to the conference theme; research and evidence-based care and, the basic principles for the design and decoration of the study room will be presented. Conclusion: There is a great challenge in producing scientific knowledge regarding issues in high-tech environments, environments which, if designed accordingly could have a healing effect on people who suffer from severe illness, their visitors and staff. References: Johansson, L., Bergbom, I., Persson, Waye, K. Ryherd, E., Lindahl, B. (2010). Analysis of relationships between the sound environment at the intensive care unit (ICU) and early signs of ICU delirium. Inter Noise: Lisbon, Portugal. Ulrich, R. (2006). Essay evidence-based health-care architecture. Lancet, 368: 538-539.

\section{Attitudes towards physician-nurse collaboration in ICU}

\section{Yasemin Ergün, Ysegul Yildirim, Turkey}

Email: yasemin3000@mynet.com

Recent research findings have found a relationship between collaboration and increased quality of care for patients, increased nurse satisfaction, and decreased cost of care. In 1986, Knaus and et al, reported treatment and outcomes in 5.030 patients in intensive care units and reported that differences in death rates related to interaction and communication between physicians and nurses. Objective: To investigate the association of collaboration between physicians and nurses in intensive care unit (ICU) Design: Prospective, descriptive, correlational study using self-report instruments. Settings: Ýstanbul and Marmara universities afflieted teaching hospital medical ICU Procedure: The jefferson Scale of
Attitudes Towards Physician-Nurse Collaboration (JSAPNC) was administered to 137 physicians and 258 staff nurses. Attitude scores were compared by gender, discipline. Main Results: JSAPNC mean scores of physician and nurse in ICU were $54.8 \pm 3.3 ; 56.1$ \pm 2.8 respectively. There is not statistically significant differences between nurse and physicians JSAPNC mean score $(p>0.05)$. No gender difference was observed. The results indicated that both groups expressed positive attitudes towards collaboration. Conclusions: Results indicated that the physicians' attitudes toward collaboration became more positive in ICU. A possible explanation for the physicians' attitude toward collaboration with nurse might be the nurses' role in intensive care unit. References 1. Coeling,H.V., Cukr, P.L.," Communication Styles That Promote Perceptions of Collaboration, Quality, and Nurse Satisfaction" Journal of Nursing Care Quality, 14, 2 (2000): 63-74. 2. Knaus, W. A., Draper E. A.,Wagner, D.P., and Zimmerman, J.E., "An Evaluation of Outcome From Intensive Care in Major Medical Centers", Annals of Intenal Medicine 104 (1986): 410 - 418. 3.Yildirim, A., Ates, M., Akinci, F. et al. "Physician-Nurse Attitudes Toward Collaboration in Istanbul's Public Hospitals," International Journal of Nursing Studies 42:4 (2005) pp. 429-437.

Autonomy and cooperation practices among critical care nurses in Cyprus

\section{Evanthia Georgiou, Andreas Pavlakis, Elizabeth Papathanassoglou, Cyprus}

Email: evagrn@spidernet.com.cy

Aim: To explore the level of critical and intensive care nurses' autonomy in Cyprus and to assess potential associations with nursephysician collaboration. Methods: The entire nursing personnel employed at intensive care units (ICUs) of public hospitals in Cyprus participated in the study $(n=184)$. Data were collected through a questionnaire consisting of 2 validated Likert-type scales, and general/demographic questions. Nurses' autonomy was assessed by the Varjus et al (2003) scale and nurse-physician collaboration by the CSACD (Baggs, 1992). Results: The majority of nurses regard autonomy and independence in their work as very important (8.33 \pm 1.48 10-point numeric rating scale). However, autonomy scores among ICU nurses were moderate $(4.23 \pm 0.9$ on a 6 -point Likert scale). ICU nurses enjoyed the strongest autonomy with regard to the value base of autonomy $(4.36 \pm 1.18)$ and a comparison of their knowledge to their action base scores showed that they had more control over matters of delivery of care than over decision making and unit operation. Nurse-physician collaboration in the decision making process was low (3.74 \pm 1.59 7-point Likert scale). A strong positive association was exhibited between autonomy and collaboration (Spearman's rho $=0.542, p=0.000$ ). Collaboration was strongly associated to satisfaction with decision making (Spearman's rho $=0.736, p=0.000$ ). Extent of nursing experience and Intensive care unit experience were important determinants of autonomy and collaboration $(p=0.001)$. Furthermore, some significant differences in the level of nurse-physician collaboration among different ICUs were observed. Conclusions: The strong association observed between teamwork and autonomy suggests that synergy rather than conflict may empower nurses and enhance their autonomy. Autonomous nursing practice and collegial/collaborative nursephysician relationships are essential for ICU nurses' satisfaction with the decision making process and are strong determinants of healthy work environments. 
Assessment of trainee nurses and practicum's tutors skills during their stay in a critical care unit

Teresa Pastor Amorós, Esther Navarro Roldán, Janina Llorens Vázquez, Monica Soler Rovira, Jonathan Rubio García, David Carbajales Álvarez, Spain

Email: in-edit-dist@hotmail.com

Aim: To assess student's training period in their last practicum in a critical care unit, their expectations, integration and autonomy; to assess tutor's abilities and pedagogic tools for teaching. Furthermore, to compare these results with other assessments from Erasmus students from four different countries and to carry out a comparison between our teaching model and their own countries's models. Methods: Thirty-two students were asked to answer a fifteen-questions poll, marking from 0 to 10 , focussing on their practicum in critical care units. Same questions were asked to Erasmus students, but adding a second poll to compare their own systems with our own protocols and procedures. Results: The assessment about these several items: knowledge, autonomy, integration, personal experience, relations between different members of the multidisciplinary team had scored $9-10$ (over 10 ) in more than $75 \%$ of these items; scores for tutors are $9-10$ in $93,74 \%$ of the trainees. Erasmus students had rated great percentage in autonomy, relationships between patients and nurses, and professional knowledge and skills. Conclusion: Positive assessments from trainee nurses encourage us to continue in our tasks as tutors, at the same time that represents a magnificent method to help trainees to improve their autonomy, knowledge and professional responsibility. Moreover it constitutes an appreciated way to integrate trainees during their practicum in a critical care multidisciplinary team. References: 1) Mulhall A. Methodological issues in nursing research. In the field: notes on observation in qualitative research. Journal of Advanced Nursing 2002 41/3:306313 2) Bernardo Carrasco J. Técnicas y recursos para el desarrollo de las clases. Ed. Rialp. Madrid 1991 3) García León FJ et al. Modelo de evaluación de las prácticas hospitalarias en enfermería con participación activa del alumnado. Enfermería Científica. 1988 79:19-25.

\section{SESSION 19: SIMULATION STRATEGIES}

\section{Simulation: right strategy for best education in critical care}

\section{Sabrina Egman, Italy}

Email: segman@ismett.edu

The role of simulation as a teaching method has long been a standard in nursing education. Dismembered latex extremities were stationed at tables where nervous students attempted venipuncture or other invasive procedures. Indwelling catheters were navigated with shaky hands into one of the two openings available. There was not a skin fold or body hair in site. The use of simulation continues to be validated as a useful teaching tool, however it has become associated with costly and sophisticated computer models that are able to deteriorate with a push of a computer key. In this day of advanced technology, we tend to think simulation is synonymous with a "high-fidelity" simulator. However, simulation can be presented within the constraints of a small budget with the same benefit of an expensive simulator.Patient situations were simulated and role playing was used to engage the learners in experiential educational opportunities which challenged the learner both logically and emotionally. Externs navigated the hospital campus using assistive devices, were fed pureed food while blindfolded, and were restrained in Geri chairs. They were able to explore actions that they would normally not chose, and adapt the beliefs and persona of other groups. We found that simulation remains an effective way to provide creative teaching strategies and allow the learner to have a little fun and a lot of interactive experience regardless of the investment in technology. Simulation as a teaching strategy does not require high tech, sophisticated, real-time patient simulators, but rather the creativity of the instructor and the engagement of the learner.

Interdisciplinary team training in critical care scenarios may improve patient safety

Tone Johnsgaard, Marit Breivik, Marit Hegg Reime, Fred Ivan Kvam, Janecke Merethe Engberg, Guttorm Brattebø, JonKenneth Heltne, Norway

Email: tone.johnsgaard@hib.no

Aim: Realistic simulation cases offer novel opportunities to ensure safe and educationally sound learning experiences. In this abstract we report students' appreciation of exposure to low-fidelity simulation of highly relevant critical care situations. The scenarios we developed emphasize practice situations requiring teamwork, communication and interdisciplinary collaboration in realistic, timecritical situations. Methods We set up interdisciplinary teams with students in their postgraduate studies in critical care nursing, 5th year medical students and 2nd year nursing bachelor program. A total of 52 teams and 262 students participated. Three teams participated in focus group interviews, which were tape-recorded, transcribed and analyzed using content analysis techniques to abstract common themes. Results Relative infrequencies of timecritical, highly stressful situations, like anaphylactic shock, call for alternative ways to train cooperation, problem solving and team communication. Focus group participants emphasized new and novel opportunities to collaborate in interdisciplinary teams as an important opportunity to learn competent performance in these simulated practice situations. Even months after their exposure to the simulation scenarios, students described in great depth and detail embodied knowledge experiences related to their own performance, and highlighted in particular the prevention of committing medication errors. Conclusion The participants reported that interdisciplinary team training in simulated environments contributed to better preparedness for real practice. Here are important implications for patient safety since such exposure can imply less trial and error, and higher appreciations of colleague's interdisciplinary contributions to improved complex problem solving. Key words Interdisciplinary team training, simulation, patient safety.

Training the emotional and psykomotor skills in simulation: is it useful in clinical practice?

\section{Gitte Tranum Johansen, Vibekek Deco, Lisbeth Rudiengaard Wolpold, Denmark}

Email: LIWO@glo.regionh.dk

Training the emotional and psychomotor skills in simulation -ls it useful in clinical practice? Background To enhance the professional skills of nurses in specialized intensive care training, Herlev, Glostrup and Gentofte Hospital established two competence courses, held at the Danish Institute for Medical Simulation in the 2nd part of the specialist education. These courses make use of the tools full scale simulation and train skills such as prioritization, implemented algorithms, cooperation and communication and debriefing. Aims We set out to evaluate the emotional and psychomotor skills trained and developed under the simulation scenarios. Results We developed a quantitative questionnaire which we administered in a pilot study. The questionnaire included seven questions in which participants were asked to rate the usefulness of different areas of simulation training on a 6-point or 4-point Likert-scale. The data was analyzed using descriptive statistic. 32 nurses educated between the 1'st of January 2007 and 1'st of august 2010 completed the questionnaire. Median age in the sample was 34,5 range $28-54$. The majority of the sample rated the simulation training as somewhat useful or 


\section{* European Critical Care Nursing: Working Together for a Better Tomorrow.}

2011 congress abstracts *

very useful. A minority rated the training as less useful or not useful at all. Implication for practice Tentatively, we conclude that in the perspective of the participating clinicians simulation training was useful in daily clinical practice. We are currently further developing the questionnaire. Among other things we will develop open-ended qualitative questions in order to investigate why some did not experience the training as useful. We hope to be able to present the results in the Spring Congress 2011. References Jean Lave \& Etienne Wenger, Situeret læring, Hans Reitzels Forlag, 2004 Vygotsky i pædagogikken, red. Ivar Bråten, Frydenlund, 2006 Tryg Patient, Læringssæt: Sikker Mundtlig Kommunikation, 2006 http:// www.personaleweb.dk/05Z4527715 www.herlevsimulator.dk Three key words Simulation Usefulness Questionnaire

\section{Using simulation to bring ECCO to life}

Melissa Pollard, Michael Nickerson, Christine Bone, United States of America

\section{Email: mpollard@ehr.org}

The American Association of Critical Care Nurses rose to the challenge of educating the next generations of critical care nurses in their creation of the Essentials of Critical Care Orientation (ECCO). This allows nurses to work at their own pace using computer modules. This has many benefits in engaging our younger generations with multimedia, allowing off shift workers the freedom to maintain their sleep cycles, and working moms to fit learning into their schedules. It is the challenge of educators to integrate the information, make it relevant, and to engage the learners through the process of completing the modules. From the start, ECCO was well received for the flexibility it allowed while providing a wealth of knowledge. Educators incorporated classroom time into the schedule to promote discussions, include case studies, and reinforce critical concepts, which was met with mixed reviews. In 2010, after an evaluation of identified ICU needs, as well as past ECCO reviews, we identified the need for more hands-on practice time as well as reviewing concepts. The suggestion was made to incorporate simulation into the program. Participants and preceptors identify areas of discomfort, skills they have not yet had the opportunity to do, and complex concepts that are often challenging to completely explain during patient care in the chaotic ICU environment. Simulation has engaged the participants in a way not previously seen. The classroom time is divided between lecture and discussion reinforcing concepts from the modules, and case scenarios were brought to life using a high fidelity computerized mannequin. Simulation has been used successful to practice multiple drip titration, hemodynamic monitoring, ventilator management, and dialysis skills. The participants have been enthusiastic and engaged and the program is highly scored on evaluations.

Nurses intubation. Videolaryngoscope McGRATH Serie $5 \AA$ Vs Laryngoscope Macintosh. A manikin study

\section{Silvia Scelsi, Piergentili Federica, Gian Domenico Giusti, Italy}

Email: s_scelsi@yahoo.it

BACKGROUND Orotracheal intubation by direct laryngoscopy is a lifesaving procedure and it is used for airways safety. This procedure can be used, after training, only by aesthesis and clinic personnel which works on extra hospital emergency setting. During intra and extra hospital emergency, it's could be better that the nurse (if there's no medical personnel) can do the intubation procedure. AIMS To value the nurse intubation ability in intensive care, using a patient simulator (Laerdal SimMan ${ }^{\mathrm{TM}}$ ), comparing the effectiveness between McGRATH serie5® videolaryngoscope and Macintosch laryngoscope. MATERIALS AND METHODS 35 nurses tried orotracheal intubation with simulator with both of devices. It was studied the time spent for the intubation and the number of attempts. RESULTS Using video laryngoscope, average the attempts of effective intubation decrease $(\mathrm{p}=0,0086)$. Valuating the time with videolaryngoscope (average 20,78 seconds) rather Macintosh laryngoscope (average 39,91 seconds), this is inferior $p$ (difference $>0$ ) $=0,0002$ CONCLUSIONS Using Mc Grath serie5® videolaryngoscope, there is a decrease of attempts and of the intubation time in the manikin.

\section{SESSION 20: MASTER CLASS}

The effectiveness of protocolised weaning for reducing the duration of mechanical ventilation in critically ill adult patients: a systematic review

Bronagh Blackwood, Peter O'Halloran, Fiona Alderdice, Karen Burns, Chris Cardwell, Gavin Laver, United Kingdom

b.blackwood@qub.ac.uk

Aim: This review assessed the effects of protocolised weaning from mechanical ventilation on the total duration of mechanical ventilation, mortality, adverse events, quality of life, weaning duration, intensive care unit and hospital length of stay. Methods: We searched the Cochrane Central Register of Controlled Trials, MEDLINE, EMBASE, CINAHL, LILACS, ISI Web of Science, ISI Conference Proceedings, Cambridge Scientific Abstracts and reference lists of articles. Language restrictions were not applied. Randomised and quasi-randomised controlled trials of protocolised weaning versus non-protocolised weaning from mechanical ventilation in critically ill adults were included. Three authors independently assessed trial quality and extracted data. Results: Eleven trials that included 1971 patients were included in the review. The total duration of mechanical ventilation in the protocolised weaning group was reduced by $25 \%$ compared with the usual care group ( $\mathrm{N}=10$ trials, $95 \% \mathrm{Cl} 9 \%$ to $39 \%, P=0.006$ ); weaning duration was reduced by $78 \%(\mathrm{~N}=6$ trials, $95 \% \mathrm{Cl} 31 \%$ to $93 \%, \mathrm{P}=0.009$ ); and intensive care unit length of stay by $10 \%(\mathrm{~N}=8$ trials, $95 \% \mathrm{Cl} 2 \%$ to $19 \%, \mathrm{P}=0.02)$. There was significant heterogeneity among studies that could not be explained by subgroup analyses based on the type of unit or type of approach (automated or professional-led). Conclusion: Protocolised weaning reduces the duration of mechanical ventilation, weaning duration and intensive care unit length of stay, but there is considerable heterogeneity among studies and an insufficient number of studies to investigate the source of this heterogeneity. Although organisational context may influence outcomes, these factors were not reported in the included studies and therefore could not be evaluated.

\section{SESSION 21: PATIENT SAFETY}

\section{Error prevention in ICU}

\section{Ruth Kleinpell, United States of America}

Email: Ruth_M_Kleinpell@rush.edu

Overview: Patient safety and prevention of errors is a major focus in healthcare and an international area of concern. Ensuring patient safety in the intensive care unit (ICU) is especially important due to the complexity of care and patient acuity levels. Aim: A literature review was conducted on error prevention in the ICU in order to identify important factors influencing patient safety in the ICU. Method: A multi-level search strategy was utilized. Literature searches were conducted (from 2000 to August 31, 2010) using OVID-MEDLINE, PubMed, and Cumulative Index to Nursing and Allied Health Literature (CINAHL) with the terms "error prevention", "ICU errors", "patient safety", "critical care", "intensive care", "safety measures" "nursing care " and "ICU safety." Results: A number of articles discuss patient safety and error prevention in the ICU, ranging from opinion and research to consensus documents. Areas for safety focus in the ICU include unplanned extubations/reintubations, disconnections 
of lines, catheters, drains; medication errors; skin breakdown, and fall/injuries, among others. Research pertinent to nursing care in the ICU has focused on nursing staffing ratios, communication and collaboration in ICU care, and specific error prevention interventions. Conclusion: A number of strategies including direct safety care measures, medication reconciliation and communication can be used to prevent errors in the ICU. The influence of nursing care on error prevention is especially significant as nurses perform the majority of patient assessment, evaluation, and care for ICU patients. This session will review key concepts related to error prevention in the ICU, highlighting nursing care implications. References Gallesio AO Curr Opin Criti Care 2008;14:700-707. Moreno RP et al. Inten Care Med 2009;9:1621-2 Reader TW et al. Curr Opin Criti Care 2007;13:732-736.

Patient safety in ICU: which factors are associated to the occurrence of nursing related adverse events?

Katia Padilha, Edzângela V. Santos, Walquíria B. Figueiredo, Natasha D. Monteiro da silva, Tiago Nery Vasconcelos, Evelize Naomi Inowe, Aline Massami Nagai, Fernanda Rodrigues, Renata Daud Gallotti, Brazil

Email: kgpadilh@usp.br

Objectives: To determine the occurrence of nursing related adverseevents(N-AE) and near-misses(N-INC) in ICUs, characterizing their nature and potential risk factors. Methods: A prospective cohort was conducted in four ICUs of a 1100-bed academic, tertiary-hospital in Sao-Paulo, Brazil, enrolling critically ill patients older than 15 years from June-August,2009. N-INC and N-AE were identified by daily monitoring of medical and nursing rounds and chart reviews. Demographic characteristics, SAPSSII-scores, length of stay, communication failures and Nursing Activities Score(NAS) were recorded. The association with $\mathrm{N}-\mathrm{AE}$ was analyzed by multivariate logistic regression. Results: Among the 134 enrolled admissions, 3932 nursing untoward occurrences were identified (mean+standarderror:29.3 \pm 2.6 occurrences). Nearly $94 \%$ were near-misses with $3682 \mathrm{~N}-\mathrm{INC}$ involving 132 admissions (98.5\%). The most common $\mathrm{N}$-INC were problems in following the daily nursing prescription (1945 incidents in $77.0 \%$ admissions); collecting/sending samples (438 incidents in $74.6 \%$ admissions); administrating medications (425 incidents in $70.1 \%$ admissions) and maintaining tubes/lines (313 incidents in $47.0 \%$ admissions). As much as $250 \mathrm{~N}-\mathrm{AE}$ were detected, affecting $54.4 \%$ of admissions. Dermatitis and pressure ulcers predominated (170 occurrences in $49.3 \%$ admissions), followed by injuries related to catheters $(n=80 \mathrm{AE})$. Nursing-staff worked overloaded (NAS mean scores: $67.7 \% \pm 1.8 \%$ ). A prolonged length of stay (>7days), nursing working overloaded (NAS $>50 \%$ ) and communication failures remained as significant independent risk factors associated to $\mathrm{N}-\mathrm{AE}$, with adjusted OR of 4.8; 3.7 and 2.8 , respectively $(p<0.05)$. Conclusions: This study was essential to determine the incidence of N-INC and N-AE in our ICUs. We identified that the N-INC were extremely frequent, corresponding to almost $94 \%$ of all nursing-related problems and involving $98 \%$ of the studied admissions. N-AE, also common, affected nearly $54 \%$ of the admissions. The occurrence of at least one N-AE presents a strong and significant association with length of stay, nursingworkload and communication issues. This study was sponsored by Fundação de Amparo a Pesquisa do Estado de São-Paulo-FAPESPGRANT2008/00955-2.
Intensive care and multidisciplinary crisis resource managemant training

\section{Id Maatman, The Netherlands}

\section{Email: I.d.maatman@icv.umcg.nl}

In the hospital, errors usually arise not due to a lack of knowledge, but due to the problem of translating knowledge into appropriate clinical actions. Decisions while performing treatment and care must sometimes be made in uncertain circumstances and under time pressure. This requires co-ordination skills and communication skills of all team members. Skills not learned or only learned in theory during regular training and usually only practised in daily practice. Skills, or rather soft skills, play a major role in $70 \%$ of all errors. Crisis Resource Management (CRM) can help you prevent such errors. We let staff, ICU nurses and physicians, experience crises with the support of CRM. We simulate a patient situation in the hospitals Skills Center using a Human Patient Simulator. Together, physicians and nurses have to solve the acute crisis. During crises, human factors and non-technical skills play the most important role. After the scenario, the training is debriefed. Video images are used to show the participants their performance. The debriefing is not equal to a judgement. Good performances and action that could be improved are discussed in a safe area. In this presentation, attention is given to the CRM principles, the design of training and the first year experience.

\section{Risk management and incident reports}

\section{Sabrina Egman, Italy}

Email: segman@ismett.edu

Rationale: The creation of a Risk Management Committee within clinical facilities can reduce the risk of errors in hospitals. The percentage of hospitals that have begun registering risk events has increased from $17 \%$ to $23 \%$. This increase indicates how clinical facilities value the importance of preventing health care risks for patients and staff in the effort to assess and improve health care management and quality. Purpose: To describe the objectives and experience of our facility regarding Clinical Risk Management education for clinicians and the protocol for monitoring sentinel events. Material e methods: ISMETT in a joint-venture with the University of Pittsburgh Medical Center has implemented a system to identify critical events. Clinicians are required to identify risk circumstances for patients and to complete a designated identification form: the Incident Report. Once Incident Reports are completed and collected, they are analyzed by a Risk Management Committee that also carries out, if necessary, a root cause analyses. The goal is to implement policies and procedures to prevent and/or reduce the recurrence of an adverse event in the hospital. Results: From 2006 to 2010 our Institute has analyzed 5000 events, stressing the need to value the problem and to enhance, if possible, the means to check and identify errors. The Committee performs a transparent job of continuous monitoring, while developing and promoting solutions for the analyzed problems possibly caused by human or system errors. In the future, a communication network system between different Risk Management Committees would favor an exchange to improve prevention strategies.

\section{Patient safety and medication}

\section{Slavica Kojanić, Marusa Brvar, Slovenia \\ Email: slavica.kojanic@gmail.com}

Pharmacotherapy can have harmful effects for patient - due either to the properties of a drug or to the possible improper procedures of its use. A medication error is any preventable event that may cause or lead to inappropriate medication or harm patient. Medication errors are a common phenomenon in everyday critical care practice. The 


\section{* European Critical Care Nursing: Working Together for a Better Tomorrow.}

2011 congress abstracts *

aim of presentation is to present and discuss some examples of medication errors from clinical practice in University Medical Center Ljubljana which were documented and are related to factors that contribute to safe medication like patient particulars, medication particulars, prescribing and ordering of drugs, drug dispensing, drug labeling and storing, procurement of equipment and devices for drug administration, rooms for preparation and administration of medication, education and training of the staff, education and informing the patients. Strategies for minimizing risk and error reporting are presented. Risk reduction strategy should focus on the organization system. Errors should be reported straightforwardly and should be openly discussed. The cause and risk factors should be identified and solutions found. There should exist forms for recording and reporting medication errors. All medication errors should be thoroughly analysed and corrective measures introduced to improve safe medication. In order to improve patient safety a regular analysis of all working processes and conditions that have a significant impact on safe medication is necessary. A positive attitude and incentive for regular and through reporting of medication errors by all staff involved in healthcare is necessary.

\section{Disturbances and disruptions during medication in an ICU}

Eva Laerkner, Annette Reinholdt, Katja Valentin Andersen, Denmark

Email: eva.laerkner@ouh.regionsyddanmark.dk

Background: There is a risk of adverse events in relation to disturbances and disruptions during medication dispensing and administration in an ICU. Aim To generate knowledge about whom or what disturbs, where and when disruptions in relation to medication dispensing and administration occur in an ICU. Methods Data where generated through a fieldwork with participant observation and interviews. The fieldwork was performed over a four week period in a nine-bed level 3 ICU. Ten nurses participated in the study. The nurses were observed during medication dispensing and administration. Results Analyzing data, five themes emerged; 1) Medication - a continuous action in ICU, 2) No place is sacred, 3) Machinery and people, 4) Dismissive or non-dismissive disturbances and disruptions and 5) a common occurrence in every day practice. There seems to be a culture in the ICU where disturbances and disruptions are a common occurrence in every day practice. The nurses are disturbed during the medication process in medication room as well as in patient room. The source to disturbances and disruptions can be categorized to be from machinery and people. Most common are disturbances caused by nursing colleagues or other health professionals. Implications for practice Nurses should be aware of their cultural practice, and create attention towards respectful work conditions between colleagues in relation to medication dispensing and administration. This knowledge could guide actions to reduce disturbances and disruptions in relation to medication dispensing and administration and thereby increase patient safety in ICU.

Health care failure mode and effect analysis of continuous renal replacement therapies: a tool to improve patient safety in clinical practice

Esteban Molano-Alvarez, Jose Angel Sánchez-Izquierdo, Juan Carlos Montejo-González, Amelia Guirao-Moya, Estrella GarcíaDelgado, Pedro Ruiz-López, Teresa Nuñez-Jiménez, Spain

Email: estemolano@gmail.com

Aim: to identify safety problems in continuous renal replacement therapies(CRRT) to establish improvement actions to reduce their effects Method: we have developed a HFMEA of process in which, seven professionals with experience on CRRT and patient safety, have been involved. Analysis sheet was used, setting out potential failures occurred in each activity, its causes and possible consequences. Subsequently, we assigned a score according to criteria of frequency, severity and possibility of detection for each of the causes. These values were used to prioritize the implementation of improvement measures. Results: the main potential failures identified were: lack of asepsis maintenance therapies as a result of awareness deficit, inappropriate prescribing treatment, and inappropriate connection to the patient, improper dosage lower than the scheduled real and incorrect withdrawal of treatment. All of them were associated with lack of training and guidelines. In addition, the early clotting of the circuits was associated with the design of the lines and failures of the catheter Conclusions: different measures are proposed to improve the process such as the development of a guideline of CRRT, conduct training sessions, improve awareness of asepsis; and implement improvements within the machine designs in order to prevent clotting of the circuit. 1.De Rosier J, Stalhandske E, Bagian J, Nudell T. Using Health care failure Mode and Effect AnalysisTM: The VA National Center for Patient Safety's Prospective Risk Analysis System. Jt Comm J qual Improv 2002; 28: 248-26 2.Oudemans-Van Strateen HM. Primum non nocere, safety of continuous renal replacement therapy. Curr Opin Crit Care 2007;13:635-637 3.Journois D, Schortgen F. Champ 7. Sécurisation des procedures d'épuration extrarénale. Annales Francaises dÁnesthesia et de Réanimation 2008;27:101-109

Immediate treatment and nursing care when patients are admitted to the ICU

\section{Eva Barkestad, Helen Hammarstedt, Sweden}

\section{Email: evabarkestad@bredband.net}

Immediate treatment and nursing care when patients are admitted to the intensive care unit A project of improvements in safety at a ICU in Sweden The critical ill patient often has multi organ failure and requires medical intervention immediately. Time is essential and the chance of survival is diminished as the number of organs involved increases. When a patient is admitted to the intensive care unit it is of great importance to ensure that the direct treatment and nursing care happens as systematically and safe as possible. We started a project with the aim to ensure that patients should have optimal care and all available medical resources at our ward at Danderyd hospital intensive care unit in Stockholm. A group with representatives from all categories of staff was interviewed about their experience and caring of the critical ill after admission to the ICU. The interviews were analyzed and three themes were found and they were: • communication/information, • organization/teamwork, • support to relatives/nearest. All staff was asked to fill in a survey with question on the above themes, the inquiry confirmed that improvement were imperative in the three categories. We made a few suggestions of changes and improvements. A new report system was established, we made the team leader more visible by altering the organization and we designated staff to care for relatives. After implementation of the new actions we made a follow up survey. The result of the follow up survey showed a slight progress in communication and information, but better organization and teamwork and also the area of supporting the nearest to cope with a critical ill relative.

\section{SESSION 22: PATIENT AND FAMILY EXPERIENCES}

Close relatives' experiences of transitions when living with a person with traumatic brain injury

\section{Åsa Engström, Siv Söderberg, Sweden}

Email: asa.engstrom@ltu.se

Background: When someone is afflicted by a traumatic brain injury (TBI) it entails a sudden change in life for their close relatives. Relatives provide the primary support system for the person with 


\section{* European Critical Care Nursing: Working Together for a Better Tomorrow.}

2011 congress abstracts *

TBI and new living patterns have to be developed by the family in order to achieve balance in the new situation. Aim. The aim of this study, was to describe transitions experienced by close relatives of people with TBI. Five close relatives, all women, who lived with or close to a person with TBI were interviewed. Method. The data were analysed in accordance with the qualitative interpretive method and performed in a series of steps in order to arrive at a description of the transition. Findings. The findings of the analysis are presented in four categories; the starting point of the transitions, transitions in pattern of daily life, transitions in relationship and transitions in social life. Conclusion. The transition was an ongoing process for close relatives due the event when the person got the TBI. The relatives could feel lonely as former friends were gone or avoided them. How the person with TBI was met by other people strongly affected how the close relatives felt. While they struggled to lessen the dependence of the person with TBI on them, they also felt anxious about how things would be if close relatives were no longer would be there for them.

Patients experience eye discomfort after treatment in an intensive care unit: a pilot

\section{Marie Eivarsson, Sweden}

Email: marie.eivarsson@sodersjukhuset.se

Background: Patients being treated in an intensive care unit is severely ill or injured. This means that they are often sedated or unconscious. These patients need good nursing to give them good care in an optimal way. There are a few studies that have investigated whether patients have eye problems related to health care in an intensive care unit. In these studies, measurement of eye performed during hospital stay. Then we come to the conclusion that patients have an increased risk of changes in the eyes of the care provided in intensive care. There is no study done which consulted on the patient believes they have eye problems after discharge from intensive care. Aim: The aim of this study is to ask patients as have been treated in an intensive care unit if they think they have eye problems after discharge from intensive care. Method: During the period April-September 2008 includes 30 patients who were over 16 years and cared for more than 4 days in the intensive care unit. The questionnaires was distributed and answered during the visit of the intensive care aftercare. There was no reduction. Result: Median age was 65 years, minimum age 33 years and maximum age 82 years. The distribution between sexes was $37 \%$ women and $63 \%$. Median duration of treatment in intensive care days were over 10 , the lowest number of care days 4 and a maximum number of 185 care days. Eight patients of 30 believe they have eye problems after treatment in intensive care.

\section{Family functioning and contribution during critical illness}

\section{Martin Nagl-Cupal, Wilfried Schnepp, Austria}

Email: martin.nagl-cupal@univie.ac.at

The aim of this study was to get a deeper insight in families and family functioning when an adult family member is admitted in an ICU and how families copes with the situation. METHODS This study was carried out using Grounded Theory Methodology according to Strauss and Corbin (1990). Data came from 22 semistructured interviews representing 11 families who had had an adult family member in an ICU. Access was assembled throughout four hospitals in Austria. RESULTS Family members use a whole range of strategies to cope with the situation. The core category is "one's own contribution" - from the point of view of the relatives - for the survival and recovery throughout the healing process of the ill family member. This contribution consists of various family care, which clearly differ from nursing care, both in their goal setting as in their implementation. In order to master the situation and go through this time, it is a "whole family effort" that is needed. The concept of "being a family" implies family care that are self-evident and given without having been asked for. "Being a family" is a negotiating process where a core of persons must be formed to take over the duties to be carried out at the bedside. This core is made of people providing the needed care. "One's own contribution" is linked with other strategies such as the permanent "being there" at the bedside, as it will be shown in the presentation. CONCLUSION For nursing practice the findings offer insights into the reality of families and, on how to assess the assumptions and the existing practice in professional care, as far as this group of people is concerned. REFERENCES Strauss, A.L., Corbin, J.L. (1990) Basics of Qualitative Research. Grounded Theory Procedures and Techniques. Sage, London.

Depression and anxiety experienced by family members of patients in ICUs

Tuba Sengul, Tuba Sengul, Aysegul Yildirim Kaptanoglu, Turkey Email: tubasen2003@yahoo.com

Objective: Hospitalization of patients represents a moment a crisis for patients and their relatives. This study was performed to determine anxiety levels and depression of relatives of patients admitted in an intensive care units (ICU). Material and Methods: This was a cross - sectional study at GATA Haydarpa ${ }^{\circ} a$ Training hospital and Marmara medical school affiliated hospital. The data collection instruments were the Turkish validation of both the hospital Anxiety and Depression Scale (HADS) and the State-Trait Anxiety Inventory (STAI). The total of both hospital sample was consisted 64 relatives of critical patients. Results: Mean age of relatives was $43.9 \pm 10.2$ years HADS mean score for anxiety and depression were $7.34 \pm 3.5$; $8.08 \pm 3.26$ respectively. Overall $54.2 \%$ and $48 \%$ of relatives of patients scored high on both anxiety and depression. Anxiety levels were STAI-State $58.59 \pm 12.47$, STAI-Trait 40.10 \pm 8.9 . These scores indicate the presence of high level of anxiety. Correlation between the HADS and the STAI were strong. There were not statistically significant difference between age and the length of the patient's hospital stay according to HADS. There were statistically significant differences between gender, level of education, degree of kinship and diagnosis $(p<0.05)$. Conclusion: The results of the study showed that the anxiety level of the relatives of patients in critical care unit is common and moderate to severe. Adequate training needed to physicians and nurses on communication with relatives of patients. Further research is needed to developed supportive measures to assist patients' family members.

Memories and health related quality of life after intensive care

\section{Mona Ringdal, Sweden}

Email: mona.ringdal@fhs.gu.se

Aim: To acquire a comprehensive understanding of patient memories from the Intensive Care Unit (ICU) -stay and their putative effects on outcome after physical trauma from a long and short-term perspective. Method: Mixed methods have been used in a multicenter study including five hospitals in Sweden. 239 adult patients were followed for five years after trauma and ICU-stay. Results: One out of four patients had delusional memories (DM) from ICU-stay. These patients also remembered more feelings of pain, panic and fear after discharged from ICU. Patients who reported DM scored lower Health Related Quality of Life (HRQoL) and more patients experienced symptoms of anxiety and depression both from a long and short-term perspective compared to those patients without such memories. From the interviews emerged both good memories of care and a gratitude for life and bad memories from a surrealistic word and an injured body. When these memories balance out, there are more possibilities to move on despite an uncertain future following the injury. Memories of being cared for and a gratitude for life seemed to make it easier to go on with life and meet this uncertain future. 


\section{* European Critical Care Nursing: Working Together for a Better Tomorrow.}

2011 congress abstracts *

Conclusion: Delusional memories correlated with lower HRQoL both in a long and short-term perspective. Good memories from care and gratitude for life make it easier to go on with life after physical trauma. References Ringdal M, Plos K, Ortenwall P, Bergbom I. Memories and health-related quality of life after intensive care: a follow-up study. Crit Care Med. 2010 Jan;38(1):38-44. Ringdal M, Plos K, Bergbom I. Memories of being injured and patients' care trajectory after physical trauma. BMC Nurs. 2008 Jun 17;7:8.

\section{Unpleasant and pleasant memories of intensive care}

\section{Karin Samuelson, Sweden}

Email: Karin.Samuelson@med.lu.se

Aim: During critical illness or injury and intensive care treatment mechanically ventilated patients may have unpleasant experiences. Following the stay in the intensive care unit (ICU) the patients' subsequent psychological well-being may be influenced by distressing memory imprints. In order to improve the patients' comfort and wellbeing during and after the ICU stay, the patients' perspective on the intensive care experience in term of memories is therefore essential. The aim of this study was to describe unpleasant and pleasant memories of the ICU stay in adult mechanically ventilated patients. Method: Mechanically ventilated adults admitted for more than 24 hours from two Swedish general ICUs were included and interviewed 5 days after ICU discharge using two open-ended questions. The data were analysed exploring the manifest content. Results: Of the 250 patients interviewed, $81 \%$ remembered the ICU stay, $71 \%$ described unpleasant memories and $59 \%$ pleasant. Ten categories emerged from the content analyses (five from unpleasant and five from pleasant memories), contrasting with each other: Physical distress and Relief of physical distress, Emotional distress and Emotional well-being, Perceptual distress and Perceptual well-being, Environmental distress and Environmental comfort, and Stressinducing care and Caring service. Among the pleasant memories, caring support, comforting nursing, respect and empathy as well as the positive attitude and demeanour of the staff predominated. Conclusion: Most critical care patients have both unpleasant and pleasant memories of their ICU stay. The pleasant memories seem to play an important role in counteracting the negative experiences. It therefore seems worthwhile to enhance positive experiences during the ICU stay, giving the patients pleasant memories which will help them move on and lessen the impact of traumatic stressors. Increased knowledge and understanding of the importance of the ICU staff in terms of demeanour and nursing care should be a major goal for all ICUs.

\section{Psychosocial nursing care in a high tech critical care unit}

\section{Lotte Abildgren Schultz, Denmark}

\section{Email: lotte.schultz@ouh.regionsyddanmark.dk}

A study of the psychosocial nursing in the encounter between the awaken intubated patient and the critical care nurse. The aim was to develop knowledge of the encounter between the intubated patient and the critical care nurse. Modern technology and new ways of treating or caring for the ventilated patient in a critical care unit, have implied a change in the patients consciousness and therefore his needs for nursing care. 44 hours of participant observation was conducted in a high technologic critical care unit in a Danish University Hospital. Three critical ill intubated patients and eight critical care nurses was included in the study. Data was analysed as one text in an Interactional Nursing Practice Theory as a theoretical framework and interpreted using a Ricoeur inspired interpretation strategy. Three themes: contact, information and interaction emerged. A divergence between the critical care nurse's intention and action in encounter may lead patient's psychosocial needs are being overlooked, despite the fact that the nurse consider the basis of care as good and professional. The findings can contribute to increase critical care nurses' consciousness of and focus on the patient's psychosocial needs during the encounter. Further research would develop a richer picture of these encounters. Keywords: Critical Care Nursing, contact, information, interactional nursing, Participatory Observation, Psychosocial Nursing Care, Ventilation Patients.

\section{SESSION 23: PROFESSIONAL DEVELOPMENTS}

The EfCCNa Delphi study: intensive care nurses' research priorities

\section{Bronagh Blackwood, Jos Latour, John Albarran, EfCCNa}

Email: b.blackwood@qub.ac.uk

Aim. This study was undertaken to identify, prioritize and reach consensus on intensive care nursing research priorities in Europe (October 2006 - April 2009). Methods. The study was undertaken using a three round Delphi technique. The panel of experts included 110 intensive care nurses, managers, educators and researchers from 20 European Critical Care Nursing Associations. Delphi round one was an emailed questionnaire inviting participants to list important areas for research. The list was content analyzed and developed into an online questionnaire for rounds two and three. In round two, participants ranked the topics on a scale of $1-6$ (not important to extremely important). Mean scores of round two were added to the questionnaire of round three and participants ranked the topics again. Results. In round one, 420 areas of research were identified. Following content analysis these were reduced to 52 research topics in 12 domains. There was a dominance of priorities in five main areas: patient safety; impact of evidence based practice on outcomes; impact of workforce on outcomes; well-being of patients and relatives; and impact of end-of-life care on staff and practice. Conclusions. The results reflect worldwide healthcare concerns and objectives and highlight topics that nurses view as fundamental to the care of critically ill patients. These topics provide a platform for future research efforts to improve clinical practice and care of patients in intensive care.

Evidence-based practice: in integration of research based knowledge into clinical practice

\section{Lisbet Narvestad Grenager, Irene Randen, Norway}

Email: lisbet.grenager@ahus.no

Background: New knowledge has to be an integrated part of all professional work to improve quality in health care. Students learn how to find and understand research and evidence based knowledge, so why do they not use this knowledge in collaboration with intensive care nurses in clinical practice? Aim: Contribute to intensive care nurses and students in the advanced intensive care nursing program, base their considerations, decisions and actions on research based knowledge (along with experience and practical knowledge). Project method: Focus group interviews with a project group and a reference group before and after implementation of a model of EBP. The unit's managers gave written consent for implementation, and Norwegian Social Science Data Services for tape recording. Participation was voluntary. The interviews will be analysed through a content analysis. Results: The project group experienced the benefit from different databases, but challenged to define keywords in the searching process. Such work was time consuming, but the speed improved by training. Uncertainty arose when the new knowledge was to be used in clinical practice. The relationship between the students and nurses was seen as more equal. Upon their new knowledge, the project group created a guide to facilitate access to databases. There has been established an EBP group in the ICU and better collaboration with the hospital's library. Implications for practice: 


\section{* European Critical Care Nursing: Working Together for a Better Tomorrow.}

2011 congress abstracts *

There is a need for further development of knowledge in critical review of science and ways of working with and attitudes towards EBP. References: Thompson DS, Estabrooks CA, Scott-Findlay S, Moore K, Wallin L. Interventions aimed at increasing research use in nursing: a systematic review. Implementation Science 2007; 2: 1-16. Tiller, T. (2004). Aksjonsforskning i skole og utdanning. Kristiansand, Høyskoleforlaget [Action research in college and education]. http:// twww.kunnskapsbasertpraksis.no [Electronic teaching program in evidence based practice].

\section{Nursing consultancy in Italian ICUs}

\section{Ilaria Cossu, Adriana Onelli, Daliel Livanu, Italy}

Email: ilaria.cossu@aniarti.it

Background The care of the patient presumes a path that can guarantee continuity of care both in the hospitals and beyond them, through the concretization of the nursing consultancy, as well as hoped for by art 13-14-15 of the ethical code of Italian nurse 2009. Particularly interesting is the nursing consultancy that critical care nurses can offer to their colleagues working in general non-ICU, to ensure a quality level of care appropriate to the critical patient. Aim to describe the characteristics of nursing consultancy and to investigate its use and distribution in Italian ICU in order to implement its use. Materials and methods Formal and informal Review of the literature (pubmed, google, nursing Italian websites) to delineate nursing consultancy characteristics. National telephonic survey through the administration of structured questionnaire, to Italian ICU inquiring into its practical application. The study was conducted starting from June to October 2010. Results Current literature tells us of some italian experiences of nursing consulting, even if not focused on specific practices of critical care area. There are differences between its use in the world and in Italy. Early results of the study, show us an almost similar situation between those who provide informal nursing consultancy and the others who don't perform it at all. Informal consultancy are especially use for technical/practical aspect. Actually only one ICU center use a formal nursing consultancy. Conclusions Italian nurse must increase this professional aspect that is an essential practice to improve the quality of care and to ensure uniformity of treatment. Dawson D, Coombs M. The current role of the consultant nurse in critical care: consolidation or consternation?. Intensive Crit Care Nurs. 2008 Jun;24(3):187-96. Fairley D, Closs SJ. Evaluation of a nurse consultant's clinical activities and the search for patient outcomes in critical care. J Clin Nurs. 2006 Sep;15(9):1106-14.

Advancing practice in critical care: a model of knowledge integration

\section{Martin Christensen, Singapore}

Email: mchristensen64@yahoo.com

BACKGROUND: It could be argued that advancing practice in critical care has been superseded by the advanced practice agenda. Some would suggest that advancing practice is focused on the core attributes of an individuals practice progressing onto advanced practice status. However, advancing practice is more of a process than identifiable skills and as such is often negated when viewing the development of practitioners to the advanced practice level. For example practice development initiatives can be seen as advancing practice for the masses which ensures that practitioners are following the same level of practice. The question here is; are they developing individually. AIM: To discuss the potential development of a conceptual model of knowledge integration pertinent to critical care nursing practice. RESULTS: In an attempt to explore the development of leading edge critical care thinking and practice, a new model for advancing practice in critical care is proposed. This paper suggests that reflection may not be the best model for advancing practice unless the individual practitioner has a sound knowledge base both theoretically and experientially. IMPLICATIONS FOR PRACTICE: Drawing on the contemporary literature and recent doctoral research, the knowledge integration model presented here uses multiple learning strategies that are focused in practise to develop practice, for example the use of work-based learning and clinical supervision. Ongoing knowledge acquisition and its relationship with previously held theory and experience will enable individual practitioners to advance their own practice as well as being a resource for others. Fulbrook, P., 2003. The nature of evidence to inform critical care nursing practice. Unpublished PhD, Bournemouth University. Rolfe, G., 1998. Education for the advanced practitioner. In: Rolfe, G., \& Fulbrook, P., Advanced Nursing Practice, pp.271-280. Oxford, ButterworthHeinemann. Scholes, J., 2006. Developing Expertise in Critical Care Nursing. Oxford, Blackwell Publishing.

Evaluation of clinical ladder in postoperative and critical care units

Siv Stafseth, A.M Storsveen, M.Austenå, T.Gjellum, L.M Alpers, H.Alfheim, M.Tøien, Norway

Email: siv.stafseth@oslo-universitetssykehus.no

Background: Clinical ladder is implemented to enhance professional development and improve quality of patient care, provide a career opportunity to bedside nurses and reduce nurse turnover(1). Rikshospitalet in Norway developed their clinical ladder in the 1990s based on models from USA, and criteria from the Norwegian Nurse Association. After completing the 5 years programme, nurses are licensed as "clinical specialist". Research shows personal accomplishment, and satisfaction and desire for professional challenge are motivators for graduation(2). The design of present study was explorative with triangulation of methods and carried out in 2009. Aim The aim of the study was to investigate nurses and nurse leaders' experience with and views on the clinical ladder programme. Results Qualitative data from interviews in focus groups with 10 nurse leaders and 15 clinical specialists. Quantitative data from questionnaire to all nurses in six units. 134 nurses answered $(55 \%)$. The nurses had a strong desire for personal and professional development, and wanted to use their new competences. Nurse leaders used clinical ladder as a tool in developing nursing quality and to recruit and withhold nurses. The leaders had to use considerable resources in integrating the programme in the units, but characterised this as an investment well worth the gains. Engagement from leaders and follow up by educational nurses are essential for the results. Implications for practice Clinical ladder can be a feasible tool for professional development in postoperative and critical care units. Professional development is a success factor for high standard in the ward. References 1.Tørstad S. and Bjørk I.T. Nurse leaders views on clinical ladders as a strategy in professional development. Journal of Nursing Management 2007;15,817-24 2.Watts M.D. Certification and Clinical Ladder as the Impetus for Professional Development. Crit care Nurs 2010;33(1)52-9.

Moral distress and structural empowerment among a national sample of Israeli ICU nurses

Freda DeKeyser Ganz, Naomi Farkash Fink,, Ofra Raanaan, Ofra Raanaan, Madeleine Bruttin, Maureen Ben Nun, Rabia Rhaliah, Julie Benbinishty, Israel

Email: freda@hadassah.org.il

Moral distress [MD] is defined as knowing the "right" thing to do but not being able to do so due to institutional constraints. High levels of MD have been found among ICU caregivers. MD has been associated with increased job dissatisfaction, burnout and worker turnover. Another work related characteristic that might be associated with $M D$ is structural empowerment [SE] or worker perception of access to sources of power in an organization (opportunity, information, 
support, resources, informal power and formal power). This association has been implied by others but has not been investigated. Aims: The aims of this study were to determine the levels of MD and SE and their association with one another among a national sample of Israeli ICU nurses. Methods: A convenience sample of 291 ICU nurses were asked to complete 3 questionnaires; a demographic and work characteristics questionnaire, the Moral Distress Scale and the Conditions of Work Effectiveness Questionnaire II. Results: Participant mean age was $37.9+9.2$ years with mean ICU experience of $9.4+8.6$ years. MD intensity [MDI] was higher than frequency [MDF]. MDI was not related to SE or any demographic or work characteristics. Significant correlations were found between MDF and general SE $(r=-.18, p<.01), \mathrm{MDI}(r=.14, p<.05)$, and age $(r=-.14$, $\mathrm{p}=.03$ ). Conclusions: Levels of MD and SE were similar to previous studies. Personal characteristics were not found to be risk factors for MDI. There is some minor evidence that MDF might be related to SE. There is a need to repeat this study with other populations and to investigate how other aspects of the work environment affect moral distress. Interventions to decrease MD could be especially aimed at younger nurses, those with a lower general feeling of SE, and high feelings of MDI.

Overview of the use of standardized language NANDA/NIC/NOC in ICUs of Madrid

\section{Jose Miguel Cachón Perez, Cristina Alvarez Lopez, Domingo Palacios Ceña, Spain}

Email: miguel.cachon@gmail.com

Aim: To determinate the experience of intensive care units nurses about standardized language Nanda/NIC/NOC Methods: A qualitative descriptive exploratory research design was employed. Sampling technical: the personal of critical care units. Critical Care Units are including on Madrid Health Service (SERMAS). Collective data tools were semistructurate interview; informed consent was obtained to tape-record them. A guide of open questions based on literature review was used. Direct testimonies of the personal (in form of daily or narrative test) were used. Collective date was done until saturated information. We use Giorgi Method to analysis the data. Using a constant comparison method, individual meaning units were sorted and resorted as categories, and then patterns, began to emerge. Patterns were combined and consolidated into common themes. Results: We interview 9 nurses, all of them about 30 years old, and we made 15 non-structured interviews. We also analyzed 3 personal letters. We identified 4 patterns: . Living integrating both sides of the same coin . Living a conceptual imposition. Living the nurses hierarchy . Living a professional development opportunity Conclusions: There are different perspectives between the standard language (NANDA) and its application in the clinical practice. These patterns appear continuously. NANDA language is sometimes perceived as a conceptual imposition. This language is far from the daily clinical experience. The use of this language is considered as a way to divide nurses into different categories. Keywords: Qualitative Research; NANDA/NIC/NOC; Nursing References: - Muller-Staub M, Lavin MA, Needham I, van Achterberg T. Nursing diagnoses, interventions and outcomes - application and impact on nursing practice: systematic review. J.Adv.Nurs. 2006 Dec;56(5):514531. - Gudmundsdottir E, Delaney C, Thoroddsen A, Karlsson T. Translation and validation of the Nursing Outcomes Classification labels and definitions for acute care nursing in Iceland. J.Adv.Nurs. 2004 May;46(3):292-302.
Design of a competency assessment checklist with failure mode effects analysis

\section{Michael Hebben, Frederique Paulus, Dave Dongelmans, The Netherlands}

Email: m.j.hebben@amc.uva.nl

Background: Maintaining and assessing nurses' competence is expected within each healthcare organization to provide quality patient care. A method to assess nurses' competence is the use of observation of performance in the actual patient care setting. To provide legitimate and objective observations, checklists can be used to assist in evaluating competency. Failure mode and effect analysis (FMEA) is a tool employed for clinical risk reduction and priority selection. In this study we evaluated the use of the FMEA method to prioritize and select items for a competency assessment checklist. Aim: Evaluate the use of the FMEA method to select items for a competency assessment checklist. Results: In a 28bed Intensive Care Unit of an academic hospital we appointed a FMEA team with 10 members including an intensivist, staff- and senior nurses. In 3 common ICU patient care processes (extubation, manual hyperinflation, endotracheal suctioning) single process steps were identified. Subsequently, failure modes were systematically identified for each of the steps and were ranked by its estimated frequency of occurrence $(\mathrm{O})$, its severity $(\mathrm{S})$, and its probability that the failure would remain undetected (D) each on a scale of 1-10. For each identified failure mode (risk priority number) RPN was calculated by multiplying the rankings for $\mathrm{O}, \mathrm{D}$ and $\mathrm{S}$. We performed a RPNs prioritization by plotting RPN on a priority matrix. For each care process the 15 steps with the highest priority numbers were included in the checklist. The majority of items (9/15) incorporated in the checklist related to infection prevention and communication. Implications for practice a prioritized competency assessment checklist can be designed for ICU patient care processes using FMEA.

\section{SESSION 24: CRITICAL CARE OUTSIDE THE ICU}

\section{Responding to antecedents of patient demise}

\section{Ged Williams, Australia}

Email: ged_williams@health.Qld.gov.au

Introduction: Literature suggests antecedent indictors to identify a deteriorating patient in the hospital ward can be measured, responded to and treated early $(1,2)$. If clinical staff are trained to identify these antecedent measures it is possible that they can raise the alarm for appropriate help early, and before further deterioration occurs. • Study Design This is an observational study of a quality improvement program. - Method This project utilised the skills of specifically trained Critical Care Nurses whose role is to respond to calls for help from nurses and junior doctors in hospital wards outside of normal business hours. Patient basic vital signs and presenting complaint as described by the ward nurse or junior doctor are collated and analysed to demonstrate trends and results from the intervention. Descriptive analysis of process improvements and rapid response/ rescue achieved through the formation of the role. $\cdot$ Results Medical and nursing staff satisfaction with the role was identified through staff surveys and focus group reports. Rapid response to antecedents and definitive treatments put in place compared to previous response regimes have improved dramatically. Permanent funding and appointment to this role is now in place for this major teaching hospital and the model is being used as a benchmark model for other Australian teaching hospitals. Conclusion Highly skilled critical care nurses have an important role in responding to patient antecedents and averting serious harm. This model of patient safety assumes the best aspects to the Nurse-at-night and ICU Liaison nurse model and 
demonstrates significant benefits to patients and the organisation generally. (1) Kause $\mathrm{J}$ et al. 2004. A comparison of antecedents of cardiac arrest, death, emergency ICU admission in Australia, NZ \& UK. Resuscitation; 62: 275-282 (2) Michael A. DeVita, et al., Findings of the First Consensus Conference on Medical Emergency Teams (MET), Crit Care Med 2006; 34:2463-2478).

\section{An investigation into the effectiveness of introducing a medical emergency team}

\section{Tom Andrews, Gerry Allen, Ireland}

Email: t.andrews@ucc.ie

Study Objectives: To examine the efficacy of a Medical Emergency Team on length of hospital stay, Length of ICU stay, In-Hospital mortality, Incidences of In-patient cardiac arrests, Admissions to ICU. Method: Six months prior to the introduction of the Medical Emergency Team patients were monitored using an established Early Warning Score (EWS) and the incidences of cardiac arrests recorded. For a six month period following the introduction the same type of patients were monitored using the EWS. The incidences of cardiac arrests were followed up for an 18 month period following the introduction of the MET. Data were analysed using Mann Whitney $\mathrm{U}$ test and Spearmani|s correlation coefficient. Results Admissions to ICU were reduced from 100 to 56 . Hospital length of stay was reduced from a median of 16.5 days prior to the introduction of MET to 10 days $(p=.002)$. Bed occupancy in ICU was reduced with no change in mortality. Prior to the MET, patients who died had an Early Warning Score of 4 compared to a score of 0.5 for those surviving $(p=.<001)$ post introduction patients dying scored 12 compared to a score of 8 for those surviving $(p=<.001)$, suggesting that sicker patients were more likely to survive post introduction of the MET. Sicker patients were ventilated (median EWS of 14) Compared to pre-MET (median score of 4). Activation of MET with a median EWS of 12 was linked with greater mortality prior to discharge $(p=.001)$. The incidences of cardiac arrest declined after the MET introduction from 50 immediately preceding the introduction 16. Conclusion The MET had a positive effect on reducing length of hospital stay, incidences of cardiac arrest and more efficient use of ICU.

\section{Reduced ICU re-admissions}

\section{Sinikka Tellervo Leppänen, Finland}

Email: Sinikka.Leppanen@ppshp.fi

Critical Care Outreach Nurse Team (CCON) is helping to reduce Intensive Care Unit (ICU) re-admissions and post ICU mortality at Oulu University Hospital (OUH). The CCON team was established at Oulu University Hospital (OUH ) in 2005-06. The team consists of 7 registered nurses, who have several years experience in Intensive Care - nursing. The CCON service is provided from 9am to $5 \mathrm{pm}$ every day. The CCON visits wards to evaluate the patients who have been transferred from the ICU on the previous day. The visit's main purpose is to evaluate a patient's current condition, and if necessary to assist and advise the ward staff about the patient's continuing care. The CCON can also be consulted about other patients whose condition the ward staff is concerned with. The CCON uses an evaluation form that is designed to guide her/him through the visit and serves to record the patient's specific information and nursing interventions performed during the visit. Early warning scoring system (EWSS) is part of the evaluation form and its purpose is to show the first signs of patient's worsening condition. Aim: To reduce ICU re-admissions and post ICU mortality of long term ICU patients. To recognize in time patients condition needing ICU treatment. To improve relationships between ICU and ward by supporting ward staff with the patient's continuing care during the first days after ICU period. Results: Readmissions to ICU was reduced from $5.5 \%$ in 2006 to less than $2 \%$ in 2007-2009. Post ICU mortality of long term patients was reduced from $24 \%$ in 2006 to $16 \%$ in 2009. Implications for practise: Nurses at wards feel supported by CCON, communications between ICU and wards has been improved. Ward nurses find easier to ask questions and guidance from $\mathrm{CCON}$.

Call for Concern (C4C): Patient and relative activated critical care outreach

\section{Karin Gerber, Mandy Odell, United Kingdom}

\section{Email: karingerbersa@hotmail.com}

The aim: The Call 4 Concern project is providing the opportunity for the patient and/or family to contact the critical care outreach team at any time to directly raise concerns regarding their health and wellbeing; concerns which may not have been detected or acted upon by the clinical team. Background: The concept of 'Call for Concern' (C4C) was inspired by Condition $\mathrm{H}(\mathrm{elp})$ system at the University of Pittsburgh's Medical Centre (UPMC) in the United States. Supporting literature reported the value of patients and relatives input into the early recognition of deterioration (Cioffi, 2000; Minick and Harvey, 2003). As the concept of patient and relative initiated CCO was unprecedented in the UK, it was felt necessary to undertake a feasibility and testing pilotroject for the C4C concept before widespread hospital implementation was contemplated. Methods: a 6 months pilot phase to evaluate the C4C service for feasibility, and effects on patients, relatives and the health care teams - a three month phase implementing the $\mathrm{C} 4 \mathrm{C}$ service onto to two surgical wards to test and evaluate the findings of the feasibility phase in preparation for expansion to all hospital wards. Results: The CCO team have received 37 C4C referrals between 1st Sept 2009 and 23 rd Sept 2010 , representing $0.5 \%$ of total CCO activity. Critical deterioration of a patient was prevented in at least two cases, and the service received positive feed back from patients and relatives. In the words of a relative, $\mathrm{C} 4 \mathrm{C}$ provided '.. . a better quality of care... and... reduces the risk of death'. References: Cioffi J. (2000). Recognition of patients who require emergency assistance: a descriptive study. Heart and Lung: 29 (4); 262-268. Minick, P. Harvey, S. (2003) The early recognition of patient problems among medical-surgical nurses. Medsurg Nursing, 12, 291-7. (This work was accepted for publication to the British Journal of Nursing).

Health and well-being after discharge from intensive care

\section{Rannveig J Jónasdóttir, Herdís Sveinsdóttir, Lovísa Baldursdóttir, Iceland}

Email: rannveij@gmail.com

Aim: Long-term outcomes after critical illness and stay in intensive care unit (ICU) have identified changes in patients' physical, psychological and social health and well-being. The aim of this descriptive, cross-sectional study is to explore health-related quality of life (HRQOL), symptoms of posttraumatic stress disorder (PTSD), memories of ICU, general health and social circumstances of patients 3 to 15 months after their discharge from ICU. Methods: Study sample $(\mathrm{N}=146)$ were all adult patients admitted for \&\#8805;72 hours from 1 March 2008 until 10 March 2009 from two ICUs in Landspitali National University Hospital of Iceland. The Short-Form $36 \mathrm{v} 2$ was used to measure HRQOL, the IES-R to measure PTSD and ICU Memory Tool to measure memories from ICU. Questions made by the researcher measured subjective health and social circumstances. Results: Response rate was $50 \%(\mathrm{~N}=73)$ and mean age $60,7 \pm 16,9$ years. Three out of eight dimensions of SF-36v2 were significantly more decreased (lower HRQOL) in the period 3 to 11 months after discharge from ICU than 12 to 15 months. $29 \%$ of patients scored highly for PTSD and they had significantly more memories of delusion and memories of feelings than patients who scored lower for PTSD. Seven dimensions of SF-36v2 were also significantly more decreased with patients with high PTSD score 


\section{* European Critical Care Nursing: Working Together for a Better Tomorrow.}

2011 congress abstracts *

than with lower score. About half of the patients said their health was much or somewhat worse than 6 months before admission to ICU and fewer were able to work outside their home now than before ICU. Conclusion: Physical, psychological and social health and wellbeing of patients who have been admitted for \&\#8805;72 hours in ICU is decreased for almost a year after discharge from ICU and continues to be decreased 15 months after discharge but to a lesser extent.

Follow up visits to an ICU: critical care nurses' experiences

\section{Åsa Engström, Siv Söderberg, Sweden}

Email: asa.engstrom@ltu.se

Background. The critical illness experience affects the ill person and their close relatives not only during the stay in an intensive care unit, but also for a long time afterwards. Follow-up visits were introduced in order to offer people the opportunity to talk about their experiences. This activity has not been studied earlier from the perspective of critical care nurses. Aim. The aim of this study was to describe critical care nurses' experiences of follow-up visits for formerly critically ill people discharged from an ICU and their close relatives. Method. Eight critical care nurses narrated their experiences of follow-up visits by formerly critically ill people and their close relatives to an intensive care unit. Data were collected during 2007 and 2008. Qualitative thematic content analysis was applied to the interview texts. Findings. The findings show that in order to feel they were doing a good job it was vital for the critical care nurses to be well prepared for the follow-up visits. It was difficult, in a positive way, to recognize formerly critically ill people when they returned looking healthy. The critical care nurses were disappointed that their former patients remembered so few real events. The follow-up visits gave the critical care nurses a new picture of how the critically illness experience influenced the former patient's everyday life during and after their stay in the intensive care unit and how it affected the lives of their close relatives. The critical care nurses felt it was valuable for them to receive feedback about the care given in form of follow-up visits. Conclusion. Receiving feedback about one's work from followup visits gives critical care nurses the possibility for to evaluate given care. Follow-up visits to intensive care units an provide them with valuable knowledge that might lead to improved nursing care.

The individualized rehabilitation project from critical care area to home

\section{Elio Drigo, Italy}

Email: edrigo@aniarti.it

Background. In severe brain damages, the earliest the rehabilitation starts the best are the outcomes. The continuity of any treatment is essential for an effective result and for the rationale use of resources. All the health carers adopting a rehabilitation strategy aim to recover the possible capabilities for the activities of daily living. Aim. To describe the benefits of the individualized rehabilitation project starting from the Intensive Care Unit (ICU), continuing into the rehabilitation wards up to the patient at home. Results. The presentation describes the process of adoption of the individualized rehabilitation project in a 3rd level of Rehabilitation Unit (RU) admitting patients with severe brain damages. The description of the problems and inappropriate organization of the care will be compared with the benefits achieved by involving the multidisciplinary team. Presentation of organizing integration instruments of nursing care in the team with results for practice: reducing hard interventions, longer hospitalization, disability complications, unclear information and involvement of the patient and his/her significant others. Implications for practice - clear definition of the objectives of the rehabilitation programme stronger interaction of all the health team members, including patient and his/her significant others - strict multidisciplinary monitoring and documentation of the on-going results of the interventions change the vision of care to a multidisciplinary action that take into consideration the possible prevention of complications or limitation of the disability resulting from the brain damage - implementation of a continuing rehabilitation programme starting from the acute settings - constantly re-considering the clinical or nursing care priorities for the patient, highlighting his/her best possible final quality of daily life References. Langhorn P, Duncan P, Does the organization of postacute stroke care really matter? Stroke, 2001; 32; 268-274

\section{Can we save more children?}

\section{Matteo Bartolomei, Stefano Musolesi, Sandretti Ilaria, Italy}

\section{Email: M.Bartolomei@118er.it}

We can say that trauma in the main killer for children. Every year in USA die more children because of traumatic events then for all other diseases together. In $2000-2006$ there have been 12.175 deaths. The aim of this paper is to find out, what can be done to prevent children's deaths an which is the nurse's role? There have been 4806 children's deaths from 1995 to 1999 in Arizona. The main causes of death were road-traffic accidents, drownings and murder. The committee said that $90 \%$ (568) deaths on the roads, $86 \%$ (161) of drownings and 36 (76) murder could have been avoided. There has been a review in Medline database literature.the articles we have reviewed indicate prevention as the first way to reduce deaths in childhood. An important role in prevention is covered by the nurses. Only a few states in the U.S.A. adopt this policy, raising awareness of children. It is noted that the states that have laws in force, regarding the use of bicycle helmets, proper use of a car seats and carry out meetings in schools have the lowest mortality rates in the nation, including Massachusetts. In Europe, if all countries had the same mortality than those with lower rates, could save young lives 3 to 4 . Health system have a central role in this new approach, integrating the prevention of childhood injuries in a broad approach to child health and development. Italy has the lowest rates in Europe for drowning, poisoning and burns in children, but regarding to falls we are in 16th place. With 87 road deaths per million inhabitants, Italy is still far from the best countries. Through the introduction of a good prevention program in our country could actually reduce mortality from trauma in children and adolescents.

\section{ABSTRACTS: POSTER PRESENTATIONS}

\section{Walking with ventilated patients}

\section{Peter Nydahl, Germany}

Email: peter@nydahl.de

Background: Immobility of patients on intensive care units (ICU) leads to several, long lasting complications. In the last years a paradigma shift took place and the light sedated, mobile and ventilated ICU patient becomes possible. Aim What impact has got walking on ventilated ICU patients? Method A pubmed search was conducted in spring 2010 with search terms mobilisation, walking, ambulation, ventilation, intensive care unit, critical care alone and in combination. Secondary hand search was added. Results 11 studies were found. The first studies which have all been performed with mixed populations of patients point out that walking with ventilated patients is feasible and safe and can have a positive influence on the ICU-stay and incidence of delirium. Walking with ventilation can contribute significantly to the quality of life, motivation and wellbeing of patients. Conclusions: A team approach is highly recommended. Video examples and an algorithm on the available evidence will be shown. 


\section{Checklist on admission to ICU patient}

\section{José Robles Carrión, Francisco Javier Vega Vázquez, Estefanía Pachón María, Maria Dolores Gonzalez Caro, Teresa Quesada Pérez, Spain}

Email: enfermeroportu@hotmail.com

BACKGROUND We can increase the quality of care and increase their safety, with a simple tool and easy to carry out, without much time to consists of the health professional: Check-list We adapted this tool to ICU, where, due to the instability of the sick, implements the safety of our patients in the process of admission to the unit, by minimizing the adverse, so we will act with good practice nurses and safer. AIM -Minimize avoidable adverse events and serious and undesirable consequences in patients -Apply new tool to help improve health care quality and safe care to provide care to patients "Improving health care as a patient safety concerns during the process of admission in ICU -Collection of data for continuous improvement tool implanted and patient safety RESULTS By analysis of recommendations implemented by various agencies (European Committee of Health, WHO, etc.), examine the processes and activities of our daily practices in which the highest percentage of incidents could happen and on this basis developed the checklist income, taking place through literature review and consensus building within the group of experienced professionals The first data set: they have reduced accidental damage and adverse effects from its application IMPLICATIONS FOR PRACTICE -It facilitates the work to new staff -Radical change in the display of customer service in her stay in ICU -Increase the safety and quality in the income -Reduces the incidence of errors and critical events unwanted experienced both staff and in the newly incorporated Tool and simple to carry out, without much time to consists of the healthcare professional REFERENCES +NHS. Seven steps to patient safety. National Patient Safety Agency, February 2004. WHO. World Alliance for Patient Safety: Forward +Programme 2005.Geneva (Switzerland): WHO; October 2004. +Manual for using the check list on the WHO safe surgery

\section{Project Zero Bacteremia}

Maria Dolores Gonzalez Caro, Juan Luis Nuñez Sanchez, José Robles Carrión, Francisco Javier Vega Vazquez, Teresa Quesada Perez, Spain

Email: enfermeroportu@hotmail.com

BACKGROUND The Spanish Agency for Healthcare Quality, in collaboration with WHO, began the project in the National Health System for the prevention of catheter-related bacteremia (CRB) in ICU The project, using a multifactorial strategy (training, implementation of protocols, acquisition of new equipment, etc.) based on the experience carried out in Michigan by Dr. Peter Pronovost of Johns Hopkins University The BRC causing a negative impact on health economic sphere, implementation of measures Bacteremia Project Zero (BZ) will decrease the rate of BRC with the consequent improvement of quality and safety. AIM . Principal: Reduction of catheter bacteremia (BRC) to ¡Ü 4 episodes per 1000 days of central venous catheter. Secondary: Promote and strengthen the safety culture in the ICUinfections RESULTS -Coverage of in-service training and on line $>70 \%$ of staff -He gets an average annual rate of $2.43 \mathrm{BRC}$ well below the initial target and even less than the national average (3.47 BRCx1000dcvc) -Decreased rates of pneumonia associated with mechanical ventilation from 11.86 to 5.70 per 1000 days of mechanical ventilation and urinary tract infections from 2.81 to $1.69 \times 100$ dias of urinary catheter, without direct intervention on both -Improved security environment, assessed by security survey before and after project implementation -Decreased morbidity and mortality related to this issue and its economic cost IMPLICATIONS FOR PRACTICE A global performance BRC rate decreases, increasing the clinical safety of our patients, and the quality of UCI BZ Applying the protocol, reduces the rates of other nosocomial infections, no specific measures to implement this Causes a change of culture in nursing care REFERENCES Literature review of the database CUIDEN, ENFISPO, CUIDATGE, PubMed National surveillance study of nosocomial infection in ICU-HELICS ENVIN http://www. seguridaddelpaciente.es/index.php/lang-es/proyectos/financiacionestudios/proyecto-bacteriemia-zero.html

Opinions of nurses working in intensive care units about euthanasia

\section{Sevim Çelik, Mine Kalkan, Ayla Gündoðdu, Hülya Topal, Turkey} Email: sevimakcel@yahoo.com

Aim: Euthanasia is not legal and much-debated practice in Turkey. The study was conducted to determine the opinions of nurses working in intensive care units about euthanasia and factors affecting these opinions of nurses. Method: This descriptive study was performed 89 nurses working in adult intensive care units at university and ministry of health hospitals of a province in Turkey. Data were collected by questionnaire and were evaluated with number, percentage, mean and chi-square tests. Results: Euthanasia was defined as willing for one's own death by intensive care nurses $(57.3 \%)$.Nurses $(59.5 \%)$ stated that euthanasia is gone against to nature, universe and God. About half (44.9\%) of nurses did not confirm to active and passive euthanasia for patients.Nurses $(85.4 \%)$ did not come across to request for euthanasia from patient or their families.Most of nurses $(80.9 \%)$ wanted to care for the terminally ill patients in intensive care units and didnot support active ( $\left.X^{2}: 9.45 ; p: 0.009\right)$ and passive euthanasia for these patients. $44.9 \%$ of nurses for themselves and $67.4 \%$ of nurses for relatives did not want to euthanasia.In addition, $58.4 \%$ of nurses did not support legalisation of euthanasia in Turkey. Conclusion:This study shows that nurses do not support the euthanasia and not confirm to active and passive euthanasia for themselves, relatives and patients. References: 1.Tepehan S,Ozkara E,Yavuz MF. Attitudes to euthanasia in ICUs and other hospital departments. Nurs Ethics 2009; 16(3),319-27. 2.Puntillo KA,Benner P,Drought T,Drew B,Stotts N,Stannard D, Rushton C,Scanlon $C$,White. C.End-of-life issues in intensive care units: a national random survey of nurses' knowledge and beliefs. Am J Crit Care 2001;10(4),216-29. 3.Van Bruchem-van de Scheur GG, van der Arend AJ, Huijer Abu-Saad H, van Wijmen FC, Spreeuwenberg C, Ter Meulen RH. Euthanasia and assisted suicide in Dutch hospitals: the role of nurses. J Clin Nurs 2008;17(12),1618-26.

Project writing workshop for nurses improves written competences and ICU nursing

\section{Hanne Juul Mikkelsen, Denmark}

Email: hannemik@rm.dk

Background: The intensive care nurse programme involves the drafting of a final written assignment (1). Nurses lack skills and practice in presenting nursing professional problems in writing. Purpose: - To provide nurses in ICU's with competences to draft a written assignment on a nursing professional problem. Method: During a three-day workshop, the nurses chose a relevant clinical problem for their assignment. This problem could be based on reflections from the daily clinical work or on theoretical knowledge challenging the quality of care at their own department (2). The nurses applied relevant quantitative and qualitative approaches to study the selected problem. The nurses had access to counselling by clinical education nurses, library assistance for literature search as well as adequate IT facilities. The nurses drafted a written report and made an oral presentation of their study. Results and implications for clinical practice The nurses reported that they had increased their competences in presenting a problem from clinical practice in writing. 


\section{* European Critical Care Nursing: Working Together for a Better Tomorrow.}

2011 congress abstracts *

Being aware of methods and written communication strategies will influence the development projects in the department in the future. Moreover, the results presented have been useful in the daily clinical work and have added to the oral presentation skills of the nurses involved in the project. Literature: 1.Cirkulære om specialuddannelsen for sygeplejersker i intensiv sygepleje. Sundhedsstyrelsens cirkulære nr. 92 af 26. juni 1997 2.Schön, D. (1987). Educating the Reflective Practitioner. San Francisco: Jossey-Bass.

Children as relatives - children visiting at the intensive care unit

\section{Kristina Louise Christensen, Denmark}

\section{Email: kristinachr@hotmail.com}

Background: Studies indicate that children would like to visit relatives at the intensive care unit. At the same time, children are rare visitors in intensive care settings (Knutsson et al. 2008). According to the studies, few intensive care units have written guidelines on chilren's visits to the department. Moreover, studies report that less than $50 \%$ of the intensive care patients receive visits from children (Knutsson, Otterberg \& Bergbom 2004). Purpose: To study how the nurse can support children between 6 and 12 years visiting at the intensive care unit. Method: A case description of an eight-year-old girl visiting her father at the intensive care unit. The theo-retical perspective on the case is two articles by Jean Piaget on development theory. Finally, recommendations to future practice are offered. Conclusion: The visit evokes different feelings in the children. It seems that preparation of the child before, during and after the visit is beneficial. The child depends on concrete actions to make logical con-clusions. Thus, the nurse has to be aware of this when she prepares the child. Verbal explanations are not sufficient to increase the child's understanding of what is happening to the relative. The nuse must take her starting point in what the child already knows and in this way help the child to gain new knowledge and understanding. References: Knutsson, S., Samuelsson, I.P., Hellstrom, A.L. \& Bergbom, I. 2008, "Children's experiences of visiting a seriously ill/injured relative on an adult intensive care unit", Journal of advanced nursing, vol. 61, no. 2, pp. 154-162. Knutsson, S.E., Otterberg, C.L. \& Bergbom, I.L. 2004, "Visits of children to patients being cared for in adult ICUs: policies, guidelines and recommendations", Intensive \& critical care nurs-ing : the official journal of the British Association of Critical Care Nurses, vol. 20 , no. 5 , pp. $264-274$.

\section{Drug compatibility chart in critical patients}

Sylvia Mónica Amorós Cerdá, Eva Pérez Juan, Maria J. Arévalo Rubert, Mònica Maqueda Palau, Bàrbara Ribas Nicolau, Spain

Email: sylvia.amoros@ono.com

Aim: Intravenous therapy is a critical aspect concerning the safety of Intensive Care Unit (ICU) patients. The aim is to elaborate a compatibility chart of drugs administered by continuous infusion. Methods: Experimental in vitro study done from January 2007 to July 2010 at University Hospital Son Dureta. The study consisted of four phases: 1. Diagnosis: detection of a lack of venous lines in critical patients and a lack of knowledge based on evidence about drug compatibility administered through Y-site 2. Selection of drugs for the study. Bibliographic research of: technical data, Handbook of Injectable Drugs and specific databases: Martindale, Micromedex and Stabilis. Selection of compatibility criteria based on bibliography 3. Laboratory work: study of physical compatibility of selected drugs reproducing continuous infusion administration through Y-site 4. Diffusion: chart elaboration; a consensus for the results was reached with the Pharmacy Department. Results: A chart with the 28 selected drugs was elaborated, including 149 double combinations. $124(83,2 \%)$ were physically compatible and 25 $(16,8 \%)$ were non-compatible Conclusions: This flow-chart, based on evidence, facilitates safe administration of intravenous infusions through Y-site and avoids adverse effects to critical patients. References: 1.Nemec K, Kopelent H, Greif R. Standardization of infusion solutions to reduce the risk of incompatibility. Am J HealthSyst Pharm. 2008;65(17):1648-54. 2.Valentin A, Capuzzo M, Guidet B, Moreno R, Metnitz B, Baurer P, et al. Errors in administration of parenteral drugs in intensive care units: multinational prospective study. BMJ. 2009;338;b814. 3.Perez Juan E, Maqueda Palau M, Arévalo Rubert M, Ribas Nicolau B, Amorós Cerdá S. Perfusiones continuas de fármacos: ¿conocemos sus compatibilidades? Nursing. 2009;27(5):54-57.

Family friendly intensive care unit. Needs and need satisfaction of critical care family members

Hanna Mayer, Martin Nagl-Cupal, Sabine Köck, Martina Mitterer, Isabella Hager, Austria

Email: pflegewissenschaft@univie.ac.at

In Austria ICUs are high specialized units, and their focus is more on the technical progress than on individual needs. In addition to this, family nursing is up to now an extensively unknown nursing concept. But families are an important support for critical ill patients. Therefore a three-step program to develop family friendly ICU's in Vienna was developed. The aim of this study was to evaluate the needs of family members at ICU's and the fulfillment of this needs. The study design was descriptive and cross sectional. For data collection a translated and adapted version of the CCFNI and the NMI was used. The survey was carried out in $15 \mathrm{ICU}$ `s of 4 participating hospitals during a time period of 6 months. The participation was voluntary and the sample size was 256 . The results show that there is a strong correlation between the importance of needs and fulfillment. The dimension empathy is high important and fulfilled well whereby the dimension getting support is least important and least fulfilled. Knowledge and communication exhibit a distance in the middle and the regulation of visiting times and the dimension being a support the major distance between fulfillment and importance. The most influencing variables were age, migration background and current quality of life. Conclusion: The main results showed that "caring - qualities" are most important and nurses fulfill these needs most time. But they are still "invisible", so they have to become an important and measurable outcome criterion of nursing quality. There is also an urgent demand for changes in basic conditions of the organization. Therefore family nursing must be a concern of the whole organization, not only an individualized concern of single nurses. Furthermore a particular attention must be paid on migrant families. More research is necessary to clarify the dimension" being support".

\section{Growing your own}

\section{Lorraine Percy, Ireland}

\section{Email: lorraine.percy@olchc.ie}

Growing Your Own Lorraine Percy RN/RCN H.Dip MSc. Nursing Applied Healthcare Management Background Nursing recruitment and retention create a challenge when caring for critically ill children. Addressing this challenge an in-house education programme was established to develop the knowledge and skills of novice nurses. The aim of the programme is to foster a sound knowledge base enabling the novice PICU nurse to develop a therapeutic and holistic approach to caring for critically ill children and their families. Implications for practice The course curriculum is divided into six modules and is delivered over a 6 month period. The student's clinical learning is facilitated by clinical support from the course coordinator and clinical facilitators. They experience a progression to care for more critically ill infants and children throughout the programme. Assessment strategies include oral presentations, written reports, completion of clinical skills book as well as two formal clinical assessments, which are based on the domains of competence from An Bord Altranais 
(An Bord Altranais, 2000). Results The successful completion of the course is seen as a stepping stone for staff to a solid career pathway within the area of Critical Care A formal evaluation identified findings such as enhancement of the nurse's confidence, knowledge and competence. For the first time in over a decade our PICU has achieved full levels of nursing staff. References: An Bord Altranais (2000) Requirements and Standards for Nurse Registration Education Programmes. An Bord Altranais: Dublin.

\section{Intensive training and mobilization at ICU}

\section{Katrine Astrup Sørensen, Astrup K, Valeur E, Denmark}

Email: katrineastrup@hotmail.com

Background: Reduced functional ability, muscular strength and cardiovascular endurance are frequent consequences of longterm hospitalization and immobilization in ICU patients. Early physiotherapeutic intervention as part of the interdisciplinary treatment is important to prevent these secondary losses of function. Intensive physiotherapy at the ICU ensures the possibility of training and mobilization several times a day. The intensive physiotherapy at the ICU; Aarhus Sygehus consists of: - Individualized training aimed to reduce loss of muscle strength and to maintain muscle function. - Mobilization to implement changes of position and to ensure a normal daily rhythm. There are 12 hours of physiotherapy on weekdays and 8 hours each day at weekends in the ICU with 6 intensive beds and 4 intermediate beds. Aim To describe the influence of intensified physiotherapeutic intervention with emphasis on training and mobilization for long-term hospitalized patients at the ICU. Results Evaluation of the physiotherapeutic intervention made by the interdisciplinary staff at the ICU revealed satisfaction with regard to training and mobilization as part of the interdisciplinary treatment. Furthermore, nursing staff and therapists from the receiving wards report smoother transition and reduced loss of function among patients, when early intensive training and mobilization is established in the ICU period of hospitalization. It enhances the possibilities for successful rehabilitation in the long term. Implications for practice Accelerated recovery for ICU patients is dependent on early intensive training and mobilization in the ICU period of hospitalization. The patients then have an improved starting point for rehabilitation when returning to their original wards.

Greek intensive care nurses' knowledge, attitudes and experiences regarding patients' rights

\section{Elizabeth Papathanassoglou, Stefania Chrysospathi, Elisabeth} Patiraki, Chrysoula Lemonidou, Cyprus

Email: e.papathanassoglou@cut.ac.cy

Aim: a) To explore the knowledge, attitudes and experiences of Greek intensive care (ICU) nurses with regard to the realization of patients' rights in critical care. b) To explore potential associations with nurses' professional characteristics. Methods: A descriptive mixed-method (focus group/ survey) design was employed at a random sample of 298 ICU nurses from the greater Athens area. Quantitative data were collected through a 36-item scale on nurses' knowledge, attitudes and experiences of patients' rights, which was validated prior to the study. Focus groups were carried out with ICU nurses. To explore associations with background variables regression analyses were performed. Results: The majority of responders (76.8\%) were unaware regarding the law on patients' rights, $79.2 \%$ regarded that patients may often or occasionally be deprived of some rights. Only $54.2 \%$ of responders regarded that informing patients about nursing procedures was necessary, and only $30 \%$ provided information to families. Sixty-nine percent of responders stated that they often or occasionally advocated for patients' rights. Coronary care (CC) nurses, $(p=0.011)$ and those in head-nurse posts $(p=0.007)$ reported higher adherence to the law. CC nurses reported more positive attitudes towards the basic principles of patients' rights $(p=0.04)$. Female nurses reported stronger emotional reactions when patients' rights were not respected $(p=0.007)$. CC nurses, head-nurses and those who attended ethics seminars reported increased involvement in the information delivery process $(p=0.03)$. Conclusion: In Greece, many ICU nurses may be unaware of the law on patient's rights, and patients' rights may often fail to be actualized. ICU nurses' experiences and attitudes towards the realization of patients' rights may diverge depending on the type of unit. In their majority, ICU nurses appear supportive of the basic ethical principles (autonomy, beneficence, non-maleficence, justice) that govern the nurse-patient relationship.

Development of an instrument for the assessment of intensive care nurses' knowledge and attitudes regarding critically ill patients' rights

\section{Elizabeth Papathanassoglou, Stefania Chrysospathi, Elisabeth} Patiraki, Chrysoula Lemonidou, Cyprus

Email: e.papathanassoglou@cut.ac.cy

Aim: To develop an instrument for the assessment of intensive care nurses' knowledge and attitudes towards critically ill patients' rights. Methods: A methodological design was employed. Focus groups were carried out with former ICU patients, ICU nurses and patients' relatives to explore the main themes of participants' attitudes and experiences with regard to the realization of patients' rights. The qualitative themes, in combination with the results of a comprehensive literature review and consultation with a group of experts, served as the basis for the development of the initial questionnaire, which was piloted at a group of ICU nurses for the assessment of its metric properties and content validity. Subsequently, the modified questionnaire was administered to a random sample of Hellenic ICU nurses $(\mathrm{N}=510)$. Assessment of component validity was carried out by factor analysis (principal components- Varimax rotation). Scoring of factors was based on the sums of scores. Associations with demographic general knowledge questions were assessed for evaluation of discriminant and convergent validity. Results: Eleven qualitative themes were taken into account for the development of 51 initial items. Factor analysis of the initial set of items resulted in five factors ( $67.45 \%$ of variance): a) adherence to the law, b) nurses' attitudes towards fundamental ethical principles, c) ethical dilemmas, d) patient information and e) nurses' attitudes towards the delivery of information. Following deletion of low correlation items, the final scale consisted of thirty-six items grouped into five subscales. Cronbach's alpha coefficient for internal consistency was 0.69. Intra-class correlation coefficients for test-retest reliability were 0.71 to 0.95 . Conclusion: There is a lack of instruments for the assessment of the realization of critically ill patients' rights. The proposed instrument has exhibited adequate psychometric properties but further testing is needed.

Pilot qualitative exploration of nurses', patients' and families' attitudes on critically ill patients' rights in Greece

\section{Elizabeth Papathanassoglou, Stefania Chrysospathi, Chrysoula Lemonidou, Cyprus}

Email: e.papathanassoglou@cut.ac.cy

Aim: To explore and to compare the experience of intensive care (ICU) nurses, of critically ill patients and of their families pertaining to the realization of critically ill patients' rights. Specifically, participants' knowledge, attitudes and views were explored. Methods: Qualitative focus group methodology. Data were collected through repetitive focus group discussions with groups (8-11 participants) of Greek ICU nurses, patients discharged from an ICU and their families. Colaizzi's analysis method was employed. Results: Qualitative themes which were common across groups were: a) lack of knowledge regarding 
legislation on patients' rights, b) obstacles towards the realization of patients' rights, c) issues pertaining to the delivery of information, d) informed consent, e) nurse-patient (-family) relationship, f) patient's privacy and dignity, g) justice, i) health care professionals' paternalistic stance. The views of all groups seemed to converge to the standpoint that the realization of patients' rights may often be unrealistic in critical care, due to the severity of patients' condition. With regard to this view, significant issues raised by all groups were shortage of staff, the architectural layout of units and lack of knowledge. Former patients regarded that information about a patient's condition and therapeutic procedures is withheld habitually, whereas, family members believed that they received adequate information, and nurses related that they hardly ever informed families and patients. Despite stating that privacy and dignity may fail to get respected, all three groups related very positive experiences with regard to nurse- patient (-families) relationships. Such relationships were the basis of trust towards nurses and formed the grounds of patients' (families') tolerance of clinicians' perceived paternalistic attitudes. Conclusion: In Greece, the realization of critically ill patients' rights may be hindered by clinicians', patients' and families' attitudes and practical issues including shortage of staff and lack of knowledge.

Interpretive synthesis of qualitative research on patients' and nurses' lived experience of critical care

Elizabeth Papathanassoglou, Maria Hadjibalassi, Foteini Vouzavali, Cyprus

Email: e.papathanassoglou@cut.ac.cy

Aim: The purpose of this synthesis of qualitative data was to reconstruct the experience of critical care from the perspective of the nurse-patient dyad, and to explore the existence of shared experiences and meanings. The focus was on the lived world of participants, on the things that affected them personally, including their perceptions of self and meaning-making. Methods: Metasynthesis of qualitative primary research, published in CINAHL, MEDLINE, Cochrane library and Psyclnfo. Inclusion/exclusion criteria were applied and methodological appraisal was carried out. Primary study data were first coded for common themes, followed by secondary coding aiming at themes reflecting shared experiences. Results: Eleven studies were included in this review. The emerging core theme was "personal transformation". Several other shared themes arose: Primacy of caring relations, suffering, bodyexperiences of the unreal, death. Nurses' and patients' perspectives on these themes were synthesized. In both patients and nurses, critical illness seems to permeate all aspects of participants' life. Participants' attunement to the values of life and human relationships appears heightened. Their perception of the future and the self appears to be affected profoundly. Restructuring their personal meanings of death is common for both patients and nurses. The experiences of caring for the critically ill and patients' stories and suffering affect the way nurses interpret themselves and the world. Conclusion: The experience of critical care may be traumatic and profound for both patients and nurses. This synthesis of interpretive data implies the possibility for growth, associated with the process of meaning-making, spirituality and authenticity in both groups. These may have implications for support interventions for either nurses or critical illness survivors. The potential benefits of the critical illness experience need to be explored in the future.
Implementation of citrate as an anticoagulant for CRRT treatment in the ICU.

Merete Kofoed, Anette Fogh, Helle Sørensen, Bettina Petersen, Anne Marie Bloksgaard, Denmark

Email: meni@geh.regionh.dk

Aim: Get a better and more stable treatment to the patients in the ICU with acute renal failure. Methods: At the CRRT treatment with heparin as an anticoagulant we experienced frequent stops in the treatment because of catheter draw, and stop in the dialyse filter. This caused many alarms great inconvenience to the patients and staff. The unforeseen and sudden stop in the treatment means that the patient lost $250 \mathrm{ml}$ blood, the CRRT therapy was not so effective and more work for the nurses. Back in 2006 we should have a new CRRT machine, and we chose among others MultiFiltration, because it gave us the opportunity to use citrate as anticoagulant. After hearing at congresses and by studytrip about Citrate as an anticoagulant in the CRRT treatment, we expected and looking forward to a significantly more stable treatment, less alarms and longer filter living time. Since the department had decided to use citrate as anticoagulant, were the department's staffs trained in this, and new instructions were prepared. Results: We started with Citrate in March 2009 and had currently been ca.28 patients who have used citrate as anticoagulant during CRRT treatment and our experience is very positive. We are seeing significantly less alarms, less uncontrolled shutdowns and more than a doubling of the filter living time, leading to a more stable treatment, and it has been possible to schedule the filter- and tubechange when it seems to be most resources in the department. Conclusion: More stable treatment and a better dialysis for the patient. Much longer filter living time. The patients need less blodtransfussions, because off less uncontrolled shutdowns. References: Fresenius Medical Care

The Nordic Association for Intensive Care Nursing Research (NOFI)

Thomas Eriksson, Ingrid Egerod, Anetth Granberg Axell, Sissel Lisa Storli, Berit Lindah, Anne Sophie Ågård, Maria Henricson, Sue Harden, Astrid Danielsen, Sweden

Email: thomas.eriksson@vgregion.se

Nordic nursing studies have a strong tradition within the qualitative approach. This approach has been applied to explore the lived experience of critical illness and intensive care therapy from the perspectives of the patient and the patient's family. In 2003, the Nordic Association for Intensive Care Nursing Research (NOFI) was established by nursing scholars from Denmark, Norway, and Sweden. The purpose of NOFI was to encourage research in intensive care nursing in the Nordic countries, to establish a network of intensive care nursing scholars, to exchange research experience and research outcomes among the Nordic research communities, and to increase the visibility of research in intensive care nursing in the Nordic countries. Although the focus was on nursing research, an interdisciplinary approach has also been encouraged with joint research and conference activities. NOFI have arranged biannual conferences for members and other individuals interested in the activities of the network. The venue has rotated among the tree Nordic countries, and the themes of the meetings have been: Intensive Care Unit (ICU) environment and acoustics, relatives of ICU-patients, ways of knowing, sedation, ethics, patient diaries, multimodal interventions, research designs, ICU-delirium, psychometrics, burns, and mechanical ventilation. Other activities have been presentations of newly completed PhD-studies in the Nordic countries. Since 2006, nursing scholars from the three Scandinavian countries have collaborated on a study of diaries written for ICU patients (patient diaries). The three countries share common values, culture and language, which has facilitated the exchange of ideas among the 
Nordic nurses. Intensive care nursing is a young domain of research. We still need to encourage more scholars within this area to increase our knowledge base, improve the ICU-experience and formalize rehabilitation of post-ICU patients. Intensive care is a collaborative practice and future research should reflect the interdisciplinary aspect of the field.

\section{Follow up in ICU}

Laila Andersen, Karin Petersen, Emilie E Holm, Louise Rasmussen, Connie Severin, Kate Pehrson, Denmark

Email: nurselaila2@gmail.com

Aim: The purpose of conducting follow up interviews is to expose emotional reactions and to offer supplementary information on the time spent in the ICU. Our aim is to help the patients deal with experiences during their hospitalisation in order to remedy any negative results. Methods: Qualitative interviews as well as a folder, which has been forwarded to the patient, offers information on frequent reactions following admittance to an ICU. The patients are contacted by phone and offered an interview together with their closest relatives three months after their admittance to the ICU. These interviews are directed by the patients and conducted by two nurses. Criteria for inclusion: Patients admitted to ICU for more than 48 hours and who are able to communicate. Our ICU is a general ward with 8 beds. We admit adults, acute as well as planned cases. Results: As this project has not yet been completed, this is a preliminary presentation. At present we have worked with this project for 13 months and have conducted interviews for 9 months. We have interviewed 29 patients, and their relatives, out of a total of 138 patients included. We have registered that $30 \%$ of the patients have experienced problems with confusion and nightmares during their stay in the unit. The physical problems have mainly comprised tiredness, sleeplessness, change in the sense of taste and hoarseness. Conclusion: On the basis of the response received from the patients and their relatives interviewed, the folder as well as the interview were of considerable value. Our Experience shows that relatives of patients, who had cardiac arrest, have special need for support If patient accept is obtained, a résumé of the interview is prepared for use in further development of nursing in the unit. References: "Patient-information for dig som nyligen vårdast på IVA anestesi- och intensivvård" developed by ICU, University Hospital in Lund, Sweden

\section{Developing a clinical guideline}

\section{Louise Simonsen, Laura Krone Larsen, Denmark}

\section{Email: tina.holm.nielsen@rh.regionh.dk}

Aim: Several international studies have shown the increased need for energy supply in patients with traumatic head injury (TBI). An audit in a neuro intensive care unit showed that patients did not reach their nutritional goal. Other studies confirm lower mortality associated with early accomplishment of individualized nutritional goals. The aim of the study was to develop a national evidence based clinical guideline in order to improve nutritional status and standardizing nutritional treatment and nursing care in a neuro intensive care unit (ICU). Method: The clinical guideline are being developed according to a template for clinical guidelines developed by the Danish Centre for Clinical Guidelines, A National Clearing House for nursing. This template prescribes focused study questions as a guide to the systematic literature search. Focused study questions were developed and the systematic literature search was conducted in June 2010 in relevant databases. The authors reviewed all included papers and a 3rd person was consulted in case of disagreement. Results: The guideline is still under progress. We expect the guideline to be approved by The Danish Centre for Clinical Guidelines during January/February 2011. The preliminary results show, that it is important to begin enteral nutrition within the first 72 hours of admission. Enteral nutrition has fewer complications than parenteral nutrition. If it is not possible to achieve nutritional goals solely by enteral nutrition, it is beneficial to combine enteral nutrition with parenteral. There is no clear answer concerning evidence on how much gastric residual volume is acceptable, without increasing the risk of aspiration. Conclusion: The final results, recommendations and implications for practice will be presented at a poster at the conference. Keywords: Nutrition, evidence-based clinical guideline, neuro intensive care unit

\section{Intensive care for the soul, not just the body}

\section{Jain Golan, Ziva Sastiel, Jain Golan, Israel}

Email: golan77@013.net.il

Background. Due to technological and medical advances, the numbers of patients suffering from chronic critical illness has risen over the years. The National Association of Medical Direction of Respiratory Care propose a definition of chronically critically ill patients as those requiring six or more hours of ventilation for more than twenty one days, (1). These patients suffer from anxiety and depression, (1). In our Intensive Care Unit (ICU), we defined this group of patients as those who were in the ICU for over 60 days, (2). Aim. Staff must rise to the challenge of giving these patients quality care. In our unit we have developed the practice of taking such patients out of hospital into the fresh air. When haemodynamically stable and fully conscious, patients are transferred to a suitable chair, attached to a mobile monitor and ventilator and accompanied by a nurse, a doctor and a respiratory technician, taken into the hospital grounds. Family members are asked to join the patient for normal social contact and to help reduce anxiety. Results. Between 2000 and 2007, nine out of twenty-seven chronically critically ill patients were taken outside. Patients and families express great appreciation of their time out of the hospital. The reconnection to the 'outside world' left them feeling optimistic about their return to health and less anxious and improved their sleep. Implications for Practice. By finding ways to meet the challenge of this newly defined group of patients we can improve their care and hopefully reduce post traumatic stress disorder suffered by many ICU survivors. References: (1 )Camhi S.L. and Nelson J.E. (2007) Vincent J-L (Editor) Yearbook of Intensive Care and Emergency medicine. Heidleberg, Springer. (2) Carmeli J. and Bursztein S. (2008) Long-Stay Critically III Patients' Characteristics and Outcomes: a Cohort study Critical Care 12 (suppl 2) p484

Nursing care to neurosurgical patients during continuous neurophysiological monitoring

\section{Monica Taffarello, Barra Vincenzo, Italy}

Email: tiziana.rececconi@startpromotion.it

AIM:Nursing care plan to optimize the quality of neurophysiological monitoring (somatosensory evoked potentials SSPE + continuous EEG) in patients suffering from post traumatic, hypoxic or ischemic coma. Methods: A prospective study after the use of neuro physiological monitoring returned in routine clinical pathways/ diagnose in acute patients with GCS $<8$. Monitoring is positioned by the physician using the 10-20 international EEG system by needle electrodes inserted in the scalp, after disinfection and fixed by sterile transparent dressing. The needle electrodes are connected to a head monitor behind the patient and connected via network. Electrical stimulus of the median nerve or the axillary one is detected on the somato-sensory cortex through averaging technique. The nursing staff must pay attention during care interventions (e.g. nursing cares, transport, environmental stimuli, and accidental disconnection of the electrodes) because they can affect the reliability of the collected data. Results: Nursing care finalized to maintaining the neuro monitoring contributes to improve the signal detection in total of cases, reducing 


\section{* European Critical Care Nursing: Working Together for a Better Tomorrow.}

2011 congress abstracts *

artefacts and allowing to keep longer monitored ( $7 \pm 5$ days). New problems emerged related to the placement of needle electrodes (sweating that leads to a poor adhesion of dressing, accidental disconnections). In $20 \%$ of cases it was reported an increase of blood pressure related to electrical stimulation that required actions by the nursing staff. Conclusion: nursing activities in preserving continuous PESS+EEG allow to obtain a better quality of neuro physiological parameters useful to patient's care. Bibliography:Bosco E, Zanatta $P$ et all. Serial somatosensory evoked potentials in severe head injury: a monitor of brain function. Acta Anaesth. Italic 59.152-173, 2008 Williams D. The elctro-encefhalogram in acute head injuries. $J$ Neurosurg Psychiatry 4:107,1941.

Interventions of nurses to prevent ventilator-associated pneumonia in intensive care units

\section{Gülten Ozden, Sevgi Sun Kapucu, Turkey}

Email: drdemirci@medusaistanbul.com

This research was conducted as a descriptive study to designate the nursing interventions aimed at preventing Ventilator-Associated Pneumonia (VAP) in Intensive Care Units (ICU). The universe of the study was the nurses working in the adult ICU's of six state hospitals and four university hospitals within the borders of the city of Ankara. Totally 290 intensive care nurses were included in the study. A questionnaire form developed by the researcher was used in the study.In the statistical analysis of the data, Student's t test was used to compare two groups, and the variance analysis method was used to compare more than two groups. It was found that the use of alcohol-based hand antiseptic and the measurement of cuff pressure were under the average among the nurses in order to prevent VAP. However, the remaining methods for preventing of VAP were applied above the average. There was a significant difference between VAP preventive measures and gender, the institution, the type of intensive care unit, the educational status, and the existence of infection control training among the nurses $(p<0.05)$. As a result of the study, it was found that nurses should be trained to prevent VAP. Suggestions were made for hospitals regarding to constitute a multidisciplinary team and organize effective training programs for infection control, to establish the procedures for prevention of VAP, and to recruit sufficient number of nurses at ICU's. 1.Centers for Disease Control and Prevention: Guideline for Prevention of HealthCare-Associated Pneumonia. (2004). MMWR, 53, 1-36. 2.Augustyn, B. (August 2007). Ventilatör-Associated Pneumonia Risk Factors and Prevention. Critical Care Nurse, 27(4), 32-39. 3.Instýtufor Clinical Systems Improvement. (May 2007). Prevention of VentilatörAssociated Pneumonia. Third Edition, 1-24. www.icsi.org.

Self-help groups at a neuro ICU

Tina Holm Nielsen, Gitte Munkebo Møller, Claus Weitze Mortensen,

\section{Denmark}

Email: tina.holm.nielsen@rh.regionh.dk

Aim: At the Department of Neurointensive care at the Neuro-Sciencecenter, Rigshospitalet in Denmark, it is a known fact that many patients die due to serious trauma, often soon after admission or after a short period of illness. Relatives are often unprepared for a swift course of events. At the Neuro ICU a self-help group was established for relatives who had lost close family members. This group started in 1995. Current research show that establishing self-help groups can have a positive effect on patients as well as relatives during times of serious illness (1). However, it has not yet been researched if participating in a self- help group at an intensive care unit can support a relative in re-establish everyday life after an unexpected death. The aim of this project is to investigate what participating in a self-help group means to the individuals' everyday life. Method: The study is based on qualitative interviews, with 6 relatives that have participated in the self-help group. In addition an interview based on focus groups is conducted with the staff that is involved in supporting the self-help group. The data is analysed using a theoretical frame of social practice as expressed in the Danish Critical Psychological tradition (2). Conclusion: Collecting data is still in progress and is expected to be completed in the Spring of 2011. The results of the analysis will be presented at the congress. References: 1. Adamsen, L.: 'From victim to agent': the clinical and social significance of selfhelp group participation for people with life threatening diseases. Scand. J. Caring Sci. 2002; 16(3):224-31. 2. Dreier O: "Psychoterapy in everyday life". Cambridge University press 2008.

\section{Hemostatic techniques in ICU}

\section{Minna Nyman, Finland}

\section{Email: minna.nyman@ppshp.fi}

Aim: After coronary angioplasty and stent placement a patient's nursing care continues at the ICU (intensive care unit). Different kinds of hemostatic techniques are used to compress the puncture site. The use of hemostatic devices requires a special know-how from a nurse. The aim of my master's thesis was to develop instructions to nurses how to treat and observe patients' puncture site after coronary angioplasty and stent placement. This kind of special knowledge does not belong to the basic education of the nurses. Instructions are needed to prevent life-threatening complications such as hematoma. Method: Instructions are based on a systematic literature review. The systematic literature review includes nine articles which were analyzed. Also manuals from the device manufacturers were used to complete the instructions. The instructions teach how to evaluate patients' femoral or radial puncture site. The instructions also include guidelines on different kinds of hemostatic techniques such as Femostop-, AngioSeal- and TR Band radial compression device and instructions how to accomplish manual pressing. Results: Nurses have found the instructions very useful and they feel more safe when they have straight guidelines how to evaluate the puncture site and how to use different kinds of hemostatic techniques and devices. Other wards in our hospital have also been interested in the instructions. Conclusions: A systematic literature review is a good way to find information to develop evidence based instructions for nursing care. The instructions help nurses to prevent life-threatening complications.

\section{Patients' safety in the intensive care unit}

\section{Efi Evangelou, Ekaterini Lambrinou, Theodoros Kyprianou,} Cyprus

Email: ee.evaggelou@edu.cut.ac.cy

AIM: The aim of this study is the identification and investigation of Nursing Sensitive Outcomes (NSOs) that can quantify patients' safety in the Intensive Care Unit (ICU). Methods: A systematic literature review was conducted aiming to identify papers in English language from 1995 up to date. Medline, CINAHL and Cochrane library were the main databases searched. Agencies, forums, associations, commissions and societies of critical care were also searched. Additional papers were searched, through the references of the papers obtained. Observational, feasibility studies and reviews were included. Results: Literature review revealed 30 NSOs that quantify patients' safety. Nursing research is limited in the field of ICU, regarding patients' safety. Existing studies seem to have methodological limitations. The majority of studies conducted in the ICU, were focused on negative patients' outcomes and adverse events including infections. Common relevant indicators among the databases searched, were found. Despite the common indicators proposed, there is a variation regarding to indicators' definitions 
and specifications. Conclusion: Patient safety is considered to be a dimension of health care quality. ICU nurses play a central role in patients' safety. Characteristics of each indicator and its relevancy to the ICU must be examined. There is a demanding need for experts' consensus on a set of NSOs that quantify and reflect nurses' role in critically ill patients' safety. References: Van den Heede $\mathrm{K}$ et al (2007). International Experts' Perspectives on the State of the Nurse Staffing and Patient Outcomes Literature. Journal Of Nursing Scholarship, 39, 4, 290-297.

Investigation of intensive care nurses views and thoughts about role and responsibilities

\section{Filizce Özel, Asiye Durmaz Akyol, Berna Sagit, Turkey \\ Email: filizce28@hotmail.com}

Aim: The aim of this study is planned for determine at intensive care nurses views and thoughts about the roles and responsibilities. Methods: The study sample included 170 nurses working in intensive care units in 4 major hospitals between July 31 2009-30 September 2009. The data in study were collected with a questionnaire form consist of two sections. First form on socio-demographic information; second form included the role and responsibilities of intensive care nurses consist of four main sections including basic characteristics of intensive care nurses, physical / mental attributes, working risks, task- power and responsibilities expression $(n=41)$. Results: Participants in the study are consisted of $\% 97,06$ women, $\% 2,94$ man;, $\% 66,47$ 'si License graduate and to work $\% 52,35$ 'i 1 -5 year. In the basic qualifications and physical / mental qualities with educational status became a significant relationship statistically and this meaningfulness is found to cause by the undergraduate and graduate groups. Care given the number of patients compared with an average of 3 fundamental characteristics of the nurses who provide care to patients, scores are significantly higher than other groups. Conclusion: Intensive care nurses related to their roles and responsibilities should have needed organization of training programs for it the development of the quality. References: 1. Anonymous. Advantages of Certification;Critical Care Nurse;Feb 2007;ProQuest Health and Medical Complete pg.36 2.Badýr A.Uluslar arasý Öneriler Ipýðýnda Yoðun Bakým Hempireliði Eðitiminin Türkiye'deki Durumu. Yoðun Bakým Hempireliði Dergisi 2004;8(2);83-887 3. Black J,Hawks JH. Medical Surgical Nursing,6. Edition,2001(159-164).

Intensive care and ethical dilemmas

\section{Filizce Özel, Asiye Durmaz Akyol, Turkey}

Email: filizce28@hotmail.com

Ethics is "the philosophy of values". Ethics can be considered as producing a systematic way for values on human actions. The term systematic analytical thinking based on ethics as a process makes it different. Health care ethics, health care system, nurses, midwives, health officers, medical technicians and physicians is the concept of interest. Ethics affects every area of health care. Historical development was respectively ethics, medical ethics, and then nursing ethics. On the basis of medical ethics and nursing ethics code constituted. The first was an ethical code of nursing was determined with Florence Nightingale. Various nursing organizations have developed codes of ethics for members. Ethical dilemmas conflict occurs in two or more ethical principles. Three areas in ethical dilemmas are euthanasia, refusal of treatment, the lack of resources in intensive care Ethical dilemmas in the intensive care unit:The primary responsibility of nurses to patients damage. Among the ethical dilemmas of intensive care unit include: *Not find enough resources for care and treatment, *Individual's economic status, *Inequality of resource distribution, *The use of advanced technological equipment, *Paternalistic approach to parenting. Which allows the consideration of orders will be made. Nursing practices are reasonable decisions. The nurse helps ethical / unit administrator for ethic dilemmas. Critical care nurses must be knowledgeable about ethical principles and practices.Accepted ethical principles is a human broad perspectives of society. Ethical principles are autonomy, nonmalefience, beneficence, justice, loyalty and integrity. According to ethical principles intensive care nurse can solve many of the ethical dilemmas. Patient-centered approach can help to solve ethical dilemmas. In addition to the ethical principles, critical care nurses are legally responsible towards the patients and nurses should have knowledge about ethical dilemmas. References: 1-Babadað K,Yoðun Bakým Birimlerinde Etik Sorunlar ve Yaklapýmlar, YBHD Yayýn Organý, Haziran,1997,(13-15) 2-BlackJ,HawksJH, Medical Surgical Nursing, 6. Edition, 2001,(53-65) 3- SoleM,HartshornJ, Overview of Critical Care Nursing,3.Edition,(25-38).

Intensive care nurse education research activities in Istanbul

\section{Özden Duruhan, Duygu Özcan, Nureddin Karaka, Mahmut} Akanman, Mukaddes Miral, Turkey

Email: ozumsun@hotmail.com

Login: Nursing education, theoretical and practical training-a program that includes education and experience,the main goal is to train individuals who can use the full potential. Professional nursing education, cultural and professional knowledge, clinical, and conceptual skills and the individual's value system is based on. ICU nurses to work with a holistic approach to safe, effective and quality care by providing patients and their families are expected to meet maintenance requirements. Therefore,advanced critical care nursing knowledge and skills is a specialty requiring knowledge and continuing education activities must be supported. Objective: In this context, we organized with the aim of postgraduate education of nurses working in intensive care units supporting education in terms of qualified manpower training, enhance the quality of health services, nursing services standards. Method: Certificate programs for nurses held in intensive care; -Intensive Care Nursing Certificate Program (Ministry of Health Turkey-2002) -Cardiovascular Surgical Intensive Care Nursing Certificate Program (Ministry of Health Turkey-2008) -Neonatal Intensive Care Nursing Certificate Program (Istanbul Health Directorate-2005, Ministry of Health 2010) -Coronary Care Nursing Certificate Program ( Istanbul Health Directorate-2007) Programs conducted by the Ministry of Health Critical Care Nursing Certificate Program Implementation Guidelines in the context of the Directorate conducted by the Certification Programs are conducted in accordance with the Implementation Guide. Instructions and guidance in the program's execution procedures and guidelines, training centers, qualifications, training program content, instructors and participants of the nature of the application how to make and how to evaluate, participants of the evaluation and certification regard to rules and procedures clearly are. Since 2007, this area of our director also arranged for the symposium are. Conclusion: With all the certificate programs and symposia, the renewal of professional knowledge and changing the direction of academic knowledge and practice are reflected in the increase provided, changes in nursing practice has been established a common language and behavior.

Perceptions of Hellenic emergency and intensive care nurses regarding changes in their professional role.

Elizabeth Papathanassoglou, Papathanassoglou EDE, Kleanthous E, Vouzavali F, Nicolaou C, Koutroubas A, Cyprus

Email: e.papathanassoglou@cut.ac.cy

Aim: To explore Hellenic intensive and emergency care nurses' perceptions regarding a) changes in their professional role, and b) the factors contributing to these changes. Methods: Qualitative focus group methodology and content analysis, with 12 participants. Results: The main theme emerged was the need for enhancement of 


\section{* European Critical Care Nursing: Working Together for a Better Tomorrow.}

2011 congress abstracts *

control over nursing practice, in order for an actual change in nurses' roles to be achieved. Participants were specific about the constituents of desired change, which included increased participation in clinical decision making, in unit operation and policy making decisions, along with enhanced overall professional autonomy. Although participants concurred that there are many advancements in clinical practice with regard to equipment and the knowledge base, they did not deem that such changes affected their professional nursing role significantly, given that nurses' participation in decision making lingered. Participants consented contentedly that the central core of nursing remains unchanged, focused on the person. Perceived advancements in clinical practice included: evidence based practice; technology, education, knowledge and clinical skills; research; and nurse-physician collaboration. The perceived reasons why these advancements failed to enhance nurses' role were lack of meritocracy; competitive relationships and lack of support among nurses; insufficient managerial support; and budget limitations. Another worth-noting theme was the conflict between young nurses entering the profession and older experienced nurses, which may suggest a tension between new and traditional nursing roles. Remarkably, participants appeared to perceive themselves as agents of change, rather than anticipating change to be brought by external influences. Conclusion: Nurses' autonomy and control over nursing practice appears to remain limited among Hellenic emergency and critical care nurses. The quality of interpersonal relationships among nurses may hinder the enhancement of their professional role. Future attempts to advance nursing practice need to target the interpersonal climate in emergency and intensive care units.

Associations between quality of interactions among health care professionals in ICU and nurses' psychological well-being in Hellas

Elizabeth Papathanassoglou, Kalafati M, Stathopoulou X, Mpouzika M, Boules P, Terzi BA, Papathanassoglou EDE, Cyprus

Email: e.papathanassoglou@cut.ac.cy

Aim: To investigate among Hellenic intensive care nurses a) satisfaction from nurse to nurse interactions and from nurse to physician interactions, b) the intensity of anxiety symptoms among ICU nurses and possible association with nurses' experience, c) possible associations between nurses' anxiety symptoms and satisfaction from interactions with ICU personnel. Methods: A correlational, cross-sectional study, with 229 randomly selected nurses employed in public and private adults' general hospital ICUs' in Hellas was performed. The intensity of anxiety symptoms was measured with Hamilton's scale. Satisfaction from interactions was measured with the Interaction subscale of Index of Work Satisfaction. The response rate was $78.6 \%$. Results: The average satisfaction score from nurse to nurse interactions was moderate to high $(5.0 . \pm 1.1)$, and from nurse to physician interactions was moderate $(4.3 \pm 1.1)$. The most frequent anxiety symptoms were anxious mood $(0.99 \pm 1.0)$, sleep $(1.1 \pm 1.2)$, sensory $(1.4 \pm 2.3)$ and muscular-skeletal $(1.8 \pm 2.4)$ disturbances. Statistically significant correlations were observed between work experience in ICU and a) muscular- skeletal symptoms (rho=0.16, $\mathrm{p}=0.012$ ), b) cognitive disturbances (rho= $0.15, p=0.022$ ). Satisfaction from nurse to physician interaction was negatively correlated with total anxiety state (tau $=-0.17, p=0.005)$, tension (tau $=-0.17, p=0.001$ ) and depressive symptoms (tau=-0.14, $p=0.008$ ). Mild negative correlations were also detected between satisfaction from nurse to nurse interaction and anxious mood (tau=$0.17, p=0.001$ ), sleep (tau $=-0.16, p=0.002$ ), muscular-skeletal (tau=-0.14, $\mathrm{p}=0.005$ ) and cardiovascular (tau=-0.14, $\mathrm{p}=0.005$ ) disturbances. Overall satisfaction from professional interactions was not a strong predictor of anxiety symptoms among ICU nurses (R2 < 0.024, Beta<-0.15). Conclusion: Nurses in Hellenic ICUs seem to be satisfied with the quality of relationships among them, as well as with physicians. Despite that anxiety symptoms are related to the degree of satisfaction from professional interactions, the latter may not be a significant indicator of ICU nurses' well- being. Further qualitative research is needed.

The use of multi-dimensional simulation to promote communication and collaboration in patient care

\section{Melissa Pollard, Michael Nickerson, United States of America}

\section{Email: mpollard@ehr.org}

No longer is the delivery of healthcare a solo endeavor. Healthcare policy makers and government regulators have published evidence that multidisciplinary teams increase efficiency and patient safety. Most medical, nursing and allied health curriculum does not prepare the healthcare provider to work with other disciplines in a team environment. Often when these different disciplines are brought together the encounter can lead to communication errors with potential impact on patient safety. Simulation allows for the training of these teams in a safe and realistic environment. Programs can be developed using actual cases encountered by clinical staff. Postscenario debriefing sessions allow for the development of inter-team communications, role clarification, identifying strengths and areas for improvement, and developing camaraderie. Our facility has used simulation for trainings in advanced cardiac life support, critical care nursing essentials, pediatric emergency team training, operating room emergency situations, obstetrical emergencies, and mock codes. This session's purpose is to discuss methods to incorporate multi-dimensional simulation activities into education programs. The objectives include sharing activities and training methods we have found successful in our institution, and discussing ways they can be incorporated into other environments to promote staff excellence, engage participation, and improve patient safety.

\section{Wean to win}

\section{Lucy Mires, United Kingdom}

Email: gheko7@hotmail.com

Background: Like many ICUs throughout the world, the battle to wean the complex patient from the ventilator continues to be a challenging yet rewarding pursuit. Approaches and techniques may vary but the steadfast foundation of successful weaning continues to be staff education and maintenance of a dynamic profile. Aim: Our aim was to captivate and educate our nursing colleagues, to create a workforce who not only understand the physiological intricacies of weaning but who will truly employ a holistic approach. The approach that we intend to adopt will incorporate staff resource packs that clearly identify and divide the different stages of the weaning patient. Group 1 - Can the patient be weaned? Group 2 - Why won't the patient wean? Group 3 - How can we help the patient to wean? Patients will be subdivided into three groups, group 1 will identify the patients readiness to wean, group 2 will address patients that have previously failed weaning attempts by focusing upon elements that may prohibit weaning and finally group 3 the complex wean will continue to build upon the previous stages whilst incorporating an individualised plan that employ techniques both physical and psychological. These packs will contain the tools required to assist with weaning and will help to prompt dynamic actions from less confident members of the team. Results: This is an ongoing project and to date we have developed weaning and exercise plans tailored to each patient. Implications Improve efficiency in effective weaning and subsequent enhancement of the patient experience. It is well documented that a reduced number of ventilator days improves patient safety, reduces length of stay and thus reduces mortality. 


\section{Swiss ICU Network Practice Development}

\section{Ursi Barandun Schäfer, Paola Massarotto, Switzerland}

Email: ubarandun@uhbs.ch

Background The "Intensive Care Nursing Network Practice Development", established in 2009, is one of three regular working groups of the Swiss Society of Intensive Care Nursing (IGIP/CISI). The clinical nursing specialists (CNS) and advanced practice nurses (ANP) involved in our group, meet three to six times yearly, and are those specialists engaged in practice development and quality management in a Swiss Intensive Care Unit. In the daily work, most of us have limited experience in our role and few opportunities to discuss strategies, methodological issues and clinical questions concerning practice development. Aim We first intended to establish a network to exchange information to break through the isolation and to make use of synergies. We are currently directing our focus on common goals and collaboration on specific topics. To obtain useful papers and tools for practice, we combine results from literature reviews with our experience as experts in clinical intensive care nursing. Results The first result, about "Intensive Care Nursing Sensitive Patient Outcomes", was presented at the annual congress of the Swiss Society of Intensive Care Nursing 2010, and is available as a paper at the IGIP/CISI-website The second result is an "Agenda for Developing Intensive Care Nursing in Switzerland". It reviews the topics of key focus to be tackled by our group, by external experts or by postgraduate students. Implications: The results of the group demonstrate the value of these efforts. Practice development is sometimes an isolated and demanding job, and the power of individual CNSs and APNs is limited. Working together in a wellorganised network helps to find optimal solutions for the nursing of critcally ill patients. In addition, it empowers the involved CNSs and APNs in every day work and supports the development of our role as practice developers, which is relatively new in Swiss critical care nursing.

\section{Levosimendan may improve the quality of life (QoL)?}

Piero Ravera, Fiorani Manuela, Micieli Concetta, Cunsolo Cristina, Depaolis Manuela, Italy

Email: ravefio@libero.it

AIM: evaluating the QoL of patients with severe cardiac insufficiency(NYHA III-IV) to whom Levosimendan is being administered during the acute phase hospitalization,before infusion and one month after treatment METHODS AND MATERIAL:perspective observation of 22 patients admitted to UOA Cardiologia2 Ospedale S.Giovanni Battista di Torino since May to September 2009.To evaluate the QoL we used the Kansas City Cardiomyopathy Questionnaire(KCCQ)before treatment and,on the phone, one month after drug administration. RESULTS: the sample was made of patients younger than average(mean age $67.35+-7.6$ ) despite of their advanced NYHA class(15 NYHA III and 7 NYHA IV). The mean KCCQ score is $42.4+-7.6$ before treatment aganist 68.3-+10.7 after treatment, with an improvementof QoL from poor to fairly good. From an analysis on aggregate data,stratified for some variables (heart transplant wait,NYHAclassand previous drug administration) we observed a pre-treatment QoL improvement:of the 17 patients with poor QoLand 5 with fairly good QoL after treatment 11 show a good QoL, 9 a firly goood one,while 2 keep having a poor QoL. CONCLUSION:Levosimendan seem to improve the QoL of patients after one month of hosptal discharge ;nevertheless the study scheme doesn't allow understanding how much the improvement depends on the drug rather than the overall optimization of the patient's treatment during hospital admission. Asideresult of this study established that methods as simple as giving a questionnaire have been able to explore specific components of the QoL of patients with cardiac insufficiency.
Multiple organ dysfunction syndrome (MODS): a state of decreased stress hormone levels and association with survival and pain

Elizabeth Papathanassoglou, Margaret Giannakopoulou, Evangelos Bozas, Sofia Mpoti, Eleni Kletsiou, Eleni Tamvaki, Iwanna Pateli, Andreas Karabinis, Georgia Fouka, Cyprus

Email: e.papathanassoglou@cut.ac.cy

AIM: Critically ill patients experience high levels of stress. The pathophysiologic sequence of stress in MODS and cellular effects of stress neuropeptides have not been studied. This research explored: a) serum levels of $\mathrm{ACTH}$, cortisol, prolactin, neuropeptide Y (NPY) and substance P (SP) in critically ill subjects and controls, b) potential association between levels of those stress neuropeptides, survival and pain. METHODS: Prospective correlational study with repeated measures. Fifty-three critically ill patients with diverse primary diagnoses and 53 -age and gender-matched healthy controls were studied. NPY and SP were quantified by ELISA and ACTH, cortisol and prolactin by cheluminense immunoassays. Pain levels were assessed by Payen and Puntillo scales. Clinical severity was quantified by MOF and MODS. RESULTS: a) statistically significant differences between critically ill and control subjects in regard with cortisol ( $p<0.0001)$, NPY ( $p<0.0001)$ and SP $(p<0.05)$ levels, b) statistically significant associations between ACTH and pain intensity levels assessed by Payen $(r=0.391, p=0.018)$ and Puntillo $(r=0.509, p=0.002)$. NPY levels were significantly lower in patients receiving analgesia $(p=0.042)$, c) lower ACTH and cortisol levels in survivors $(p<0.05) d)$ at the day of least severity, a significant association between SP levels and MOF was observed $(r=0.29$, $p<0.05)$. CONCLUSION: a) Despite the fact that NPY and SP are stress neuropeptides, their levels appear to be decreased in MODS patients, b) the observed association between stress neuropeptide levels and survival in critical illness needs to be explored further, c) bedside measurement of selected neuropeptides in the future may provide an estimation of pain in uncommunicative patients. REFERENCE(S). 1. Papathanassoglou ED et al. Multiple organ dysfunction syndrome pathogenesis and care: a complex systems theory perspective. Nursing in Critical Care, 13(5):249-59, 2008

One year education program by the employer - an impetus to professional development of experienced ICU nurses

\section{Marita Ritmala-Castrén, Finland}

Email: marita.ritmala-castren@hus.fi

Background Critical care nursing requires a wide and deep competence in nursing, and understanding of patients' medical condition. Nurses' competence and education level are associated with patient safety and outcomes of ICU care. Finland has no official critical care nursing education after the basic nursing degree. All further education is in the hands of nurses' employers. Aim The aim of this program was to make visible and improve the skills of experienced ICU nurses. The purpose was to educate and motivate them to actively participate in development of nursing practices and to remain committed to their work. Results Helsinki University Hospital developed a one year education program (40 cepts) for experienced critical care nurses. It included 36 hours of critical care medicine and nursing lectures, introduction to evidence based practice and literature search, practical training, lecturing practices and preparation of a thesis. 18 nurses from 11 different critical care and step down units participated in the course in 2009. Their mean age was 38,7 and mean ICU experience was 9,1 years. They demonstrated a significant increase in their theoretical knowledge measured with BKAT-7. Their understanding of evidence based practice clearly grew while doing the thesis, which included a literature review and a small practical study. The program's implementation was carefully evaluated by the supervisory group. Participants rated 


\section{* European Critical Care Nursing: Working Together for a Better Tomorrow.}

2011 congress abstracts *

the theoretical education as excellent, means of lectures $7,4-9,8$ (scale 1-10). Practical training in a different unit was considered "the best part of the program". Implications for practice It is important to invest in motivating and professionally advancing the experienced nurses as much as the new ones. The experienced nurses possess vast experience which needs to be made visible and refined for the good of the patients by offering nurses knowledge and tools to further develop and improve nursing practices.

Coping behaviors of haemodialysed patients' families in a private clinic in Gauteng.

\section{David Mphuthi, Ronel Pretorius, South Africa}

Email: ditaba.mphuthi@wits.ac.za

Background: Renal failure continues to be the most fast growing disease. This is due to the fact that most people suffer from pre disposing diseases like hypertension and diabetic. Patients with renal failure then have to be looked after by the families after their dialysis session. Family has to adapt to the changes that this disease brings to them. Problem: The family with a member on haemodialysis is faced with challenges. Amongst others, the roles change if the bread winner is the sufferer as well as financial constraint regarding the daily living. Aims: To identify and explain the coping behavior used by families caring for a haemodialysed patient. To explore the identified coping behavior of the families using focus group interviews. Methodology: The study followed a non-experimental descriptive and explanatory mixed method approach. It was divided into two phases being quantitative and qualitative. Sampling: All inclusive sample of the Fresenius Medical Care. men and women Data collection: Data was collected by questionnaire (F-COPES scale) and was analyzed with the help of the statistician Ethics: The University of North-West has given the approval for the study to be conducted. The participants were informed of their rights, protection as well as anonymity. Participants also gave consent before being included in the study. Conclusion: The results of the study will be disseminated upon completion. The size of the study may call for continuity so that the results can be generalized. AUER J (2002) Dialysis-a-family matter. A personal tribute to the relatives of kidney patients. European Dialysis and Transplant Nurses Association (EDTNA) 28(3):141-144, July. CRESWELL JW (2003) Research Design. Qualitative, Quantitative and Mixed Methods Approaches. 2nd Ed. Thousand Oaks: Sage Publications. GUILLEMENT A, NOLLET A, ROLLAND F \& ROY S (1988). The consequences of end-stage renal disease on the family. The role of the social worker. EDTNA-ERCA.

\section{Diary and follow-up dialogue - pieces in the puzzle of recovery}

\section{Karen Nilsson, Susanne Berner, Iben Hertz, Denmark}

Email: nilssonkaren@hotmail.com

BACKGROUND: After critical illness psychological problems with varying degrees of amnesia, distorted memories, sleep disturbance and nightmares are well documented and it is shown that intensive care units (ICU) patients have an increased risk for development of post traumatic stress disorder. AIM: To help patients to reconstruct and understand their hospitalization during critical illness and thereby achieve a better rehabilitation. Reading the diary along with the follow-up dialogue contributes to assemble the patient's puzzle and illuminate experiences from the ICU. Thereby possible reduce or prevent the development of post traumatic stress disorder. RESULTS: Patients appreciate their diary, it helps them to understand how sick they have been and by the dialogue with the follow-up team they gain a sense of coherence of their incomprehensible experiences. The ICU learns from patients' experiences and encourages improving care and policies. IMPLEMENTATION IN PRACTICE: October 2008 the care staff started writing of electronic diaries to intensive care patients who were critically ill and mechanically ventilated three days or more. The follow-up team had ongoing bedside teaching of colleagues along with study sessions with colleagues. Patients receive their diary in the ward after discharge from ICU, the follow-up team visits the patients and through dialogue clarifies the patient's memory from their stay in intensive care; dreams, delusional memories and hallucinations. After discharge the patients are offered telephonic contact by 3,6 and 12 months and are invited to visit the ICU. Patients are encouraged to fill in a questionnaire regarding quality of life (FS 36) for later research. GOALS FOR THE FUTURE: Promote cooperation across departments and thereby facilitate patients' psychological rehabilitation. Key words: Diary; Follow-up dialogue; Psychological rehabilitation. Jones C, Capuzzo M, Flaatten $\mathrm{H}$, et al 2006. "ICU diaries may reduce symptoms of posttraumatic stress disorder." Intensive Care Medicine; 32(suppl 1):S144.

Greek intensive care unit nurses' clinical decision making: documentation and classification

Karra Vassiliki, Papathanassoglou EDE, Lemonidou C, Giannakopoulou M, Cyprus

Email: e.papathanassoglou@cut.ac.cy

Aim: To describe the types of clinical decisions made by Greek Intensive Care Unit (ICU) nurses. Method: A qualitative design was used. Twenty-three purposefully selected ICU nurses with 2.512 years of critical care experience (6-14 years of overall nursing experience) participated in the study. Data were collected via an original daily documentation form where participants reported their decision making. Participants' daily decisions and judgments during patient care were recorded at real time. Data collection continued until saturation was reached. Daily entries were analyzed by qualitative content analysis in order to classify nurses' decisions according to their type, degree of emergency, and dependencyindependency in relation to medical orders. Results: Participants' clinical decisions were classified into eight categories: 1) Assessment (choosing methods to identify the subjective and objective cues of a clinical situation), 2) Diagnosis (nursing or medical), 3) Prevention (anticipating potential implications that require nursing or/and multiprofessional care and taking appropriate action), 4) Intervention (choosing among interventions focused on patient's needs and clinical status), 5) Communication (with patients and their relatives), 6) Clinical Information Seeking (by asking an experienced colleague or searching the literature), 7) Setting Clinical Priorities (making specific choices taking into account time pressure, uncertainty and clinical emergency) and 8) Referral to the health care team (deciding which observations warrant communication to other members of the health-care team). Conclusions: In Greek ICUs, nurses may make manifold, complex, patient-centered decisions in line with the steps of the nursing process, even though they may not realize it most of the time. Further research is needed to assure generalizability of these findings. References: Bucknall (2003). The clinical landscape of critical care nurses' decision-making. J Adv Nur;43(3):310-19. Thompson (1999). Qualitative research into nurse decision making: factors for consideration in theoretical sampling. Qualitative Health Res;9(6):815-828.

Rectal ulceration as a complication of the use of the flexi-seal fecal management system: a report of 2 cases

Francisco Javier Carmona Monge, Irene Álvarez Angorrilla, Felisa Rodríguez Ruiz, Elena Sánchez Aguado, Spain

Email: Eli.Casado@smith-nephew.com

Background: The Flexi-Seal Fecal Management System is a device designed to offer an improved care to the critical care patient in the presence of fecal incontinence. There is evidence showing the safety and effectivity of the device. There's growing evidence of adverse 


\section{* European Critical Care Nursing: Working Together for a Better Tomorrow.}

2011 congress abstracts *

events related to its use. Aim: We report two cases of critical care patients who developed complications associated with the use of the Flexi-Seal FMS. Results: both patients (a 71 years old male and a 67 years old female) carrying a Flexi-Seal FMS presented significant rectal bleeding, after short times with the device placed, with no further complications. In both cases, rectal ulceration consistent with the shape and location of the device was confirmed with a colonoscopy. Conclusions: The Flexi-Seal FMS is an effective method for managing liquid stools in the critical care enviroment. We should take special care of this device, being extremely important to release the pressure of the retention balloon from time to time and to place the collection bag in a position where not excessive traction is produced in the rectum. The Flexi-Seal is useful to manage fecal incontinence, improving patient well-being and reducing the complications. It is necessary to improve the knowledge related to the complications of its use in critical care patients.

Nursing workload analysis in a polivalent ICU using the Nursing Activities Score

Francisco Javier Carmona Monge, Gloria Rollán Rodríguez, Cristina Quirós Herranz, Isabel Cerrillo González, Ana Jara Pérez, Rosana Alameda Varela, Sonia García Gómez, Montserrat Martínez Lareo, Spain

Email: Eli.Casado@smith-nephew.com

Aim. The aim of the present study is to evaluate the nursing workload in an medical ICU using the Nursing Activities Score, and determine the real nursing workload of groups of patients with different pathologies. Method Prospective descriptive study performed in a 12 bed ICU, from October 2007 through december 2010. Nursing workload was measured using the Nursing Activities Score for each patient during the study period. All patients were followed up for all of their ICU stay until they were discharged, for the present study data of days 1 to 30 of stay were used. Results A total of 913 patients were admitted to the unit during the study period. The median length of stay (LOS) was 3.08 days (1.92-7.13). Regarding the admission diagnosis, the most frequent was acute coronary syndrome $(41.3 \%$; median LOS 2.3 [1.8-3.2]) and acute respiratory failure (22.5\%; median LOS 8.8 [3.7-21.5]). Mean nursing workload for each groups was 50.5 (SD 13.1) for coronary patients and 66.9 (SD 15.1) for respiratory patients. There was a statistically significant difference in nursing workload between the groups $(p<.05)$. However, there was no statistically significant difference in nursing workload between the 30 days of follow up. Conclusions Analyzing the nursing workload for the different pathologies of the patients admitted to the ICU will help improve human resources allocation. The different types of patients will have different nursing workloads and this must be taken into account to adjust the nursing staff in the ICU to the real care needs of the patients admitted to that ICU. Knowing the nursing workload in an ICU will allow us to plan the necessary staff, to maintain adequate health care quality and control the costs for the care of critically ill patients.

\section{Diary as a friend}

\section{Eva Laerkner, Anette Djursaa, Katja V Andersen, Denmark \\ Email: eva.laerkner@ouh.regionsyddanmark.dk}

Background:A diary, written by the nurse to the patient, during the period of treatment at ICU, can help the patient and relatives to get a post ICU period understanding of what really happened. Partly or fully remembered experiences can be put together in context. Aim. To review how the patients and relatives use the diary, and decide whether the diary has an influence on recovery after ICU.Methods. A questionnaire with 3 open questions is handed over to the patient, together with the diary when the patient leaves ICU. Patients and possible relatives (if patient is deceased) are expected to return the form to ICU. 9 patients and 4 relatives have returned the form. Results:Unclear or missing memory. Patients remember very little, or nothing from ICU. The diary is used to reconstruct the course of events during ICU admission. The Diary as a friend: Patients report that the diary is used as a book of facts, truth and comfort. The diary is regarded as a friend, since it is written with personal warmth and commitment. Over time, the patients keeps returning to the diary - as the need for answers arises. Diary as a source of knowledge:Relatives report that the diary contains important information of what really happened during a dark and stress full time.Implications for practice:Both patients and relatives expresses gratitude that a personal diary has been written.We believe that the feedback from patients and relatives - clearly indicates that the diary has a positive influence on the post ICU life.

\section{Implementation of an electronic patient journal (EPJ) in ICU}

\section{Hanne Helm Harvey, Denmark}

Email: haharv@rm.dk

Aim: Documentation of nursing and nursing actions electronically, so there are more clarity, context of care- and treatment- progress and better safety to the patient. That documentation is current and newest available wherever the patient is in treatment. Methods: Before the rollout of EPJ, there was set up a group to find all words used to describe the observations and issues that may arise, when a patient is hospitalized in ICU. What descriptions will be necessary in our daily documentation of nursing? What observations do we have of the respective patient groups, drainage, aspiration etc.. Then we clarified which words our unit uses the most to describe nursing problems, so everyone will feel that they document sufficient. There should creates nursing problem sheets with health professional content (SFI) on the various issues and a general one for the current nursing status. All employees at the unit got 2 hours introduction to EPJ and at start, two weeks with 24 hours support available in the unit. Results: It has been with great advance that there was made a lot of preparation creating SFIs, so it was possible to document sufficient on nursing problems and current status from day one. SFIs and conceptual clarification have roughly been consistent with user's earlier documentation tools and therefore made it easy. It has afterwards been necessary to adjust some SFIs and also make more. Conclusion: It was very difficult to imagine how the SFIs and the structure of EPJ would function before it actually was in use, but it was well received in the unit and everyone have documented from day one. There are still oncoming new SFIs and updating of earlier SFIs in the unit, while other groups and sections also have demands for documentation.

Incidence and risk factors for catheter-associated urinary tract infection in a medical ICU

Francisco Javier Carmona Monge, Ana Jara Pérez, Montserrat Martínez Lareo, Sonia García Gómez, Cristina Quirós Herranz, Gloria Rollán Rodríguez, Isabel Cerrillo González, Rosana Alameda Varela, Spain

Email: Eli.Casado@smith-nephew.com

Aim: The aim of the present study is to evaluate the incidence of catheter-associated urinary tract infection and the risk factors for its development in a medical ICU. Method Prospective study performed in a 12 bed ICU (October 2007 - December 2009). Data regarding all urinary catheters placed, urine cultures obtained and risk factors of interest for the study. Results During the study period a total of 584 urinary catheters were placed with a total of 8019 days of catheterization and 35 urinary tract infections, with and incidence of 5.11 infections for 1000 days of catheterization. $63.1 \%$ of the infections were caused by Gram negatives, $29.7 \%$ by Gram positives and $7.2 \%$ by fungus. The risk factors for the development 
of a catheter-associated urinary tract infection identified with the univariate analyses were female sex $(\mathrm{OR}=3 ., 85$, IC95\% 1.92-7.71), mechanical ventilation $(\mathrm{OR}=3.14$, IC95\% 1.19-8.25), length of stay (OR=3.97, IC95\% 1.71-9.25), use of catecholamines (OR=3.19, IC95\% 1.19-8.56) and blood transfusions (OR=3.69, IC95\% 1.43$9.57)$. All identified risk factors were then introduced in a maximum binary logistic regression model, eliminating in each step the variable with a non significant $p$ value. The final model was composed by length of stay in ICU (OR=1.04, IC95\% 1.02-1.05), female sex $(\mathrm{OR}=6.53$, IC95\% 2.85-14.97) and having an arterial catheter placed (OR=2.62, IC95\% 1.03-6.66). Conclusions Incidence of catheter-associated urinary tract infection in our unit is higher than that obtained in Spanish ICUs (ENVIN-UCI 2009). It is necessary to have an adequate care of the urinary catheter to try to diminish the amount of infections for 1000 days of catheterization. It is important to be aware of the risk factors of developing urinary tract infections in critical care patients to establish guidelines that account for this factors and point to the adequate care of patients with any of the risk factors identified in the present study.

Monitoring depth of anaesthesia of abdominal hysterectomy patients, ASA 1 and 2 - a quality development project

Tabita Gallacher, Anny Sørensen, Annemarie Vanger Larsen Mikkelsen, Denmark

Email: tabgal@rm.dk

Background: Currently, depth of anaesthesia is clinically assessed at the Regional Hospital of Randers, using blood pressure, pulse, cilia reflex, pupil changes, sweat outbreaks, etc. In recent years several devices have been developed to monitor the depth of anaesthesia that can be used as a supplement to the clinical assessment of the depth of anaesthesia by recording the patent's EEG during anaesthesia. Today we do not utilise depth of anaesthesia monitoring. Studies indicate it can actually reduce the consumption of anaesthesia agent by up to $19 \%$. We have elected to use "Spectral Entropy" from GE Healthcare in our project. Those of our patients who undergo abdominal hysterectomies are given a pre-operative epidural of Bupivacain $0.25 \%$. Patients under general anaesthesia are sedated with Propofol and Ultiva ${ }^{\circledR}$ and intubated. Post-operative patients are given fentanyl, Dehydrobenzperidol $\circledast$ (DHB) and Toradol ${ }^{\circledR}$. Aim The aim of the project is to determine if the monitoring of the depth of anaesthesia of abdominal hysterectomy patients with epidural catheters, given general anaesthesia with Propofol and Ultiva ${ }^{\circledR}$, reduces the consumption of anaesthesia agent and benefit the patient. Results The project results are expected by the middle of March 2011 Implications for practice Approximately 60 patients will be used in the project, of which half will be given anaesthesia according to the current guidelines, while the rest will have their depth of anaesthesia monitored using Entropy Monitoring from GE Healthcare. References Nielsen, Jane Stab et al. 2007. Søvndybdemonitorering - en medicinsk teknologivurdering. The National Board of Health, Denmark. www.sst.dk Toft, Palle and Barlev, Norman. 2008. Bispektral indeks til forbedret anæstesigivning og postoperativ opvågning". I: Ugeskrift For Læger 170/39. 22. Sep. 2008 pp 3050-3053.

Finding the way with GPA (guided patient autonomy)

\section{Gili Benari, Israel}

\section{Email: gilib255@gmail.com}

We need our patient's informed consent in order to treat him. That is a legal requirement. We need our patient's understanding and participation in giving informed consent in order to give him the best treatment that is right for him. That is a professional requirement. But what does it really mean? Too often we give our patients the relative information, without taking the time to find out how much was understood. When a patient is in ICU, his and his family's ability to process information in a state of stress is seriously compromised. Yet many times they are expected to decide between several options, being given facts and statistics, but with no guidance from experience, not enough support in reaching the decision that will best serve them and their needs. We know from the literature that even doctors, sick with an ailment within their area of expertise, voice the need for a caregiver to help them decide what treatment is right for them. A patient and his family, without prior knowledge need this guidance even more. It is our responsibility to find the balance between paternalism on the one hand and giving facts to the patient, sometimes mistaken as giving the patient total autonomy. Neither is no longer relevant, or at least should not be the standard of care. Patient surveys show that sometimes they want the right to abstain from deciding, sometimes they do not even want the facts. True autonomy is to understand the unique needs and wants of the patient and cater to these needs. Objectives: - Understanding the processing of stressful information. - Reaching an understanding with our patients - empowering them and us. Key words: Autonomy, communication, ethical care.

Validation of the Norwegian Behavioral Pain Scale (BPS) in mechanically ventilated and sedated patients

\section{Brita Fosser Olsen, Tone Rustøen, Norway \\ Email: britafo@fredfiber.net}

Aim Mechanically ventilated patients often experience pain, and both sedation and respirator treatment makes the pain difficult to assess. Observations of patients' behavior has proven to be satisfactory for assessing pain in this patient group and various observation tools have been developed with varying validity. The purpose of this study was to translate one of the tools, BPS, into Norwegian, use it in an intensive care unit and to further test the inter-rater reliability and feasibility of the tool. Methods This pilot study was descriptive and observational. BPS contains an assessment of patients' facial expression, upper limbs, and compliance with ventilation. Two nurses observed and assessed pain in the same patient (sedated and mechanically ventilated) at the same time without communicating with each other using BPS, both at rest and at turning. Then, the feasibility and the inter-rater reliability were examined using Kappa statistics and Intraclass correlation coefficient. Results The BPS was translated into Norwegian using back- forward procedure (BPS-N). 50 nurses used BPS-N, and it shows good feasibility and was sensitive to changes in pain behavior. Inter-rater reliability varies on the different dimensions in the tool, and the agreement was lower during turning of the patient compared with the patient at rest. Facial expressions stand out as an area with the lowest inter-rater reliability. Conclusion BPS-N is a promising tool showing good feasibility in sedated and mechanically ventilated patients. Inter-rater reliability is varying and some parts of the BPS must be developed further before it can be recommended in daily clinical practice. References Payen JF et al (2001). Assessing pain in critically ill sedated patients by using a behaviorial pain scale. Critical Care Medisin, 29, s 22582263.

Implantation of the Nurse Tutor for training and teaching staff in an ICU

Raquel Sanchez Torres, Nazaret Briones Lopez, Olga Corral Pedraz, Maria Del Carmen Garcia Martinez, Hector Gomez Garcia, Dolores Hernandez Diez, Angel Rodriguez Encinas, Carlos Hernan Moreno, Spain

Email: reichelgrin@yahoo.es

AIM: To explain the role of the "nurse-tutor" figure in order to improve the quality of the patient's care and the nurses' safety. MATERIALS AND METHODS: The criterion to select this figure is based on a 


\section{* European Critical Care Nursing: Working Together for a Better Tomorrow.}

2011 congress abstracts *

profile created by the hospital's Nursing Department, which must include: - A nurse with proven experience in ICU and up to date training. - Teaching and training skills. This figure should preferably be in the morning shift and should not be assigned care loads, except for service needs and those inherent in the supervision of newly hired staff. This figure performs the following functions: 1 . Tutoring and training of the newly hired personnel, within and outside the ICU, and their supervision during the shift. 2. Review protocols and create working groups. 3. Support the rest of the staff with the new techniques and clinical practice. 4. Training/organization of intra/extra ICU courses. 5. Support, on demand, in other hospitalization areas that work with patients who require the same cares that the critically ill patients. 6. Evaluation of the newly hired staff. CONCLUSIONS: We think that the creation of this figure can lead to a care of higher quality and a higher safety for the patient, and it will help to improve the safety in the professionals' nursing practice. We consider that it is necessary to make an assessment through a survey to evaluate this project in a more exhaustive way.

European perspective for prevention of VAP - does it work? Implementation of European perspective for prevention of VAP in ICU for surgical patients in University Medical Centre Ljubljana, Slovenia

\section{Valerija Svilar, Iza Repe, Dragica Emeđi, Slovenia \\ Email: svilarster@gmail.com}

In the ICU for surgical patients in University Medical Centre Ljubljana, Slovenia nurses conducted a randomized study of two groups of patients. The first group receive the nursing care under European perspective for preventing VAP: patients have a subglottic drainage endotracheal tube connected to the pump for subglottic aspiration, adequate measure in endotracheal tube cuff is performed at 4-6 hours, a closed suction system is used. The second group is control group: patients without a subglottic drainage endotracheal tube, without adequate measures in cuff and with open suction system. At the time of adoption on department all patients are intubated and sedated. They need mechanical ventilation. Ventilator circuits are changed after 7 days, patients have active humidification, mouthcare with Chlorhexidine at least 3 times per day and, if possible, with a raised head for $30-45$ degrees. The purpose of the study is to show the importance of compliance with the European perspective for the prevention of VAP. Role of nurses in health team is described. By preventing VAP we can significantly raise the quality of the nursing care and safety for patients, we can reduce patients length of stay in the ICU, health care costs and mortality. References: Torres A, Ewig S, Lode H, Carlet J. Defining, treating and preventing hospital acquired pneumonia: European perspective. Intensive Care Med (2009) 35:9-29. Coffin S E et al. Strategies to prevent Ventilatorassociated pneumonia in acute care hospitals. Infect Cintrol Hosp Epidemiol 2008; 29:31-40.

\section{Preventing ventilator-associated pneumonia: nursing care}

\section{Raquel Fernández, Marisa Arencibia, Joana Pírez, Spain}

Email: raquelfdezj@yahoo.es

AIM: Ventilator associated pneumonia (VAP) is the first cause of mortality amongst the nosocomial infections in intensive care unit (ICU). The aim of this study is to asses the efficacy of nursing care following the European Care Bundle to prevent VAP. METHODS: Retrospective cohort study in an 18 bed medical and surgical intensive care unit in which the ratio nurse: patient is 1:2.5. Data were collected from a first group of patients before introducing the protocol since April to June 2009. The protocol was set up, and 8 months later data from a second group of patients were collected between April and June 2010. The protocol included: strict hand hygiene using alcohol solution, bed rest elevation at $30^{\circ}-45^{\circ}$, regular gastric aspirations to avoid gastric overdistention, regular mouth washing with chlorhexidine, intermitent subglotic suctioning, regular checks of cuff pressure, non-ventilatory tubing circuit change every week, stress ulcer prophilaxis, sedation vacation, enteral feeding, active humidification in patients receiving mechanical ventilation for over 3 days and training of nursing staff. We assessed the efficacy of the changes introduced. RESULTS: In the first group we recorded data from 118 patients, 72 received mechanical ventilation (MV) (768 days), we obtained an incidence of 26.04 VAP in 1000 days under MV. In the second group after the protocol was established and used properly in $93.8 \%$ of the cases, we assessed 176 patients. MV was used in 111 patients (902 days), we obtained a result of 11.09 VAP in 1000 days of MV. CONCLUSION: The introduction and correct use of a strict protocol reduces the incidence of VAP, with a reduction of total ICU and hospital stay, then reducing costs.

Patient safety in intensive care units: the role of nursing workload in the development of nosocomial infection in intensive care units

Katia Padilha, Edzângela V. Santos, Walquiria B Figueiredo, Natasha D. Monteiro da silva, Taigo Nery Vasconcelos, Evelize Naomi Inowe, Aline Massami Nagai, Fernanda Rodrigues, Renata Daud Gallotti, Brazil

Email: kgpadilh@usp.br

Objective: To determine the association of nursing workload in the occurrence of Nosocomial Infection (NI) in critically ill patient. Methods: This prospective cohort study was conducted in four ICUs of an 1100-bed academic, tertiary-hospital in Sao-Paulo, Brazil, enrolling critically ill patients older than 15 years-old, during two consecutive months in 2009. NI was identified by daily monitoring of medical and nursing rounds, chart reviews and laboratory database consultation. Demographic characteristics, organ dysfunction (SOFAscores), length of stay (LOS), communication failures and Nursing Activities Score(NAS) were also recorded. The association with $\mathrm{NI}$ was analyzed by multivariate logistic regression. Results: During the study period, 134 admissions were included, corresponding to 128 patients ( 69 males). The mean age, ILOS, NAS and sofa-scores were respectively (mean+standard error) $51.4+1.7$ years; $9.6+0.8$ days; $67.7 \% \pm 1.8 \%$; and $4.7+0.3$ points. Considering that in our ICUs each nursing-professional was responsible for 2 patients, the detected NAS score indicates an excessive nursing workload. Among the 134 enrolled admissions, 52 developed $\mathrm{NI}$ during their ICU stay $(38.8 \%)$, predominating issues related to central venous catheters and artificial ventilation. A prolonged LOS (>7days), nursing working overloaded (NAS $>50 \%$ ), higher organ dysfunction (SOFA $>4$ points) and communication failures remained as significant independent risk factors associated to $\mathrm{NI}$, with adjusted OR of $7.1 ; 6.3 ; 5.0$ and 2.6, respectively $(p<0.05)$. Conclusion: Nursing personnel shortage and excessive nursing workload are considered facilitating conditions to the occurrence of preventable adverse events. Nosocomial infections are important quality indicators, being especially deleterious when affecting critically ill patients. Although nursing staff problems and $\mathrm{NI}$ are both frequent in ICU settings, a clear association has not been determined in prior investigations. This study showed that nursing team working overloaded was an important independent risk factor for the development of $\mathrm{NI}$ among our ICU patients, being only surpassed by a prolonged length of stay. 
Open ICU: opinion of patients, relatives and professionals

Cristina Quirós Herranz, Rosana Alameda Varela, Gloria Rollán Rodríguez, Isabel Cerrillo González, Sonia García Gómez, Ana Jara Pérez, Montserrat Martínez Lareo, Spain

\section{Email: fjcarmona@fhalcorcon.es}

Aim: Understand the opinion of the patients, relatives and professionals regarding the flexibility of schedules of visits to the ICU and the participation of relatives in the care of patients in a ICU. Methodology: Descriptive transversal study developed in a polivalent ICU. An anonymous survey was distributed among patients, relatives and professionals of the unit to know their opinion about the current visitor policy, the possibility of introducing greater flexibility of schedules and the participation of the family in the patient's basic care. Results: 252 surveys completed. $90 \%$ of total responded agree that greater flexibility in the visitor policy would be positive. $95 \%$ of patients and relatives think that this change would not affect the work of the professionals, whereas $68 \%$ of the professionals believe that it would influence their work. $80 \%$ of the relatives and $54 \%$ of the professionals think that there would be benefits in the participation of the relatives in the patient's basic care. Conclussions: The three groups agree that an open ICU would be beneficial for the patient. Nevertheless some professionals are reluctant to accept a radical change of the current policy. There exists a significant statistical difference between the opinion of patients and relatives and that of professionals with regards to the influence that this change would have in the daily functioning of the ICU. The participation of the family in the daily care of the patient would improve inter-group relations, but some relatives recognize not to be totally qualified for this task.

\section{Changing times: a new role, the zone leader}

\section{Samantha Jane Boullin, United Kingdom}

Email:sam_boullin@hotmail.co.uk

Background: In 2000 the United Kingdom (UK) Department of Health published "Comprehensive Critical Care: A Review of Adult Critical Care Services" (Department of Health, 2000). This review led to modernisations of adult critical care services including the growth and development of critical care beds. In 2007 a 13 bedded general intensive care unit in a large UK university teaching hospital expanded to 21 critical care beds. Through large scale recruitment, nursing skill mix was inevitably diluted leading to increased organisational pressures for the shift leader. Aim: To ensure continued high quality service delivery the term zone leader (ZL) was developed to identify the role of a nurse (Band 5 post foundation course and above) in a particular zone of the unit ( 3 in total) who would assist the nurse in charge following the unit expansion. Results: No formal training was provided to ZLs who alongside their patient allocation would support admissions/discharges, junior nursing staff and the organisation of staff breaks. One year post introduction of ZLs, a study day was facilitated by the lead clinical educator to debrief nurses following the role change, provide team building and communications exercises and celebrate achievements. Most revealing was the insight for both ZL and shift leader into each other's respective roles. Implications for practice: As critical care units continue to increase in scale, this presentation shares lessons learned from the introduction of the ZL's dynamic role in a UK intensive care unit and demonstrates important recommendations for practice including future $\mathrm{ZL}$ education. References: Department of Health, (2000) Comprehensive Critical Care: A Review of Adult Critical Care Services, London: HMSO.
Critical to success: lessons learned to maintain standards of care in the face of recruitment challenges.

\section{Samantha Jane Boullin, Amanda Barnes, Chrissie Guyer, United Kingdom}

Email:sam_boullin@hotmail.co.uk

Background: The challenges posed for unit leaders to recruit appropriately experienced nurses in expanding critical care units are ever increasing. A 13 bedded general intensive care unit in a large United Kingdom (UK) university teaching hospital recently expanded to 21 critical care beds. A major recruitment drive generated nurses from diverse backgrounds, many with little critical care experience, including newly qualified nurses. The units pre-existing nurse induction was initially utilised. However, knowledge and skill deficits were evident leading to the conclusion that the current induction and mentorship programme was no longer appropriate for the large number of new, inexperienced recruits. Aim: To promote recruitment, retention and ensure patient safety an urgent and extensive review of orientation and training was conducted by the unit Matron, a Lead Clinical Educator and the Lead Nurse for Practice Development. Results: Through dynamic leadership a new induction and clinical support strategy was created. Fundamental changes included a new starter assessment to direct learning needs, an early focused education programme, and mentorship workshops to develop guidelines for support. Furthermore bedside clinical guidelines were developed, reflecting and encompassing key government and hospital trust drivers. Implications for Practice: Currently UK preregistration nurse education does not provide the requisite level of knowledge and skills to be a competent practitioner in critical care (Dawson, 2006). This presentation shares experiences from a rapidly expanded unit and highlights how changes implemented have resulted both in positive evaluations from nursing staff and an increasing cohesion in an ever evolving team, that may be key in future staff retention. References: Dawson, D. (2006). The art of nursing: a hidden science? Intensive and Critical Care Nursing. 22(6), 313-314.

Heart transplantation versus ventricular assist device (VAD): evaluation and analysis of nursing workload and average days of ICU stay

\section{Gianluca Dilettoso, C Anelli, A Crespi, D Balestri, Italy}

Email: gianluca.dilettoso@tin.it

Aim: More and more often, patients are admitted to hospital to undergo complex operations such as the insertion of a VAD (ventricular assist device) or a heart transplantation. The aim of this study was to analyse the nursing workload and the average days of ICU stay relating to this type of patients. Methods: The analysis was untertaken in the Cardiothoracic Intensive Care Unit of Niguarda Ca'Granda Hospital, Milan, between April 2008 and March 2009. The study was carried out on patients who underwent transplant and the insertion of a VAD. The NEMS (Nine Equivalents of nursing Manpower use Score) scale has been used to measure the nursing workload and to plan daily nursing staff allocation at the individual patient level. Results:VAD: 6 patients who underwent surgery with a mean age of $49 \pm 2.4$ years and $12 \pm 4.16$ average days of hospitalization obtained a mean value of $36.86 \pm 4.2$ on the NEMS scale. Hearth transplantation: 38 patients who underwent surgery with a mean age of $44.8 \pm 10.7$ years and $9.16 \pm 3.6$ average days of hospitalization obtained a mean value of $31.23 \pm 3$ on the NEMS scale. The population analysed was made up by a sample of 762 patients admitted to the ICU. Conclusion: Collected data underline a higher nursing workload and a more conspicuous number of days of ICU stay necessary to assist these patients than the average NEMS values concernig in-patients who undergo other cardiothoracic operations (mean value of $28.17 \pm 1.1$ on the NEMS scale and $6.28 \pm 3.1$ average days of hospitalization). References: 
- Guccione A, Morena A, Pezzi A, lapichino G; The assessment of nursing workload; Minerva Anestesiol. 2004,70(5):411-6 • Miranda DR, Moreno R, lapichino G; Nine equivalent of nursing manpower use score(Nems); Intensive Care Med.1997;28(7):760-5

Minimally invasive cardiac surgery using the heart port technique: nursing in critical care

\section{Gianluca Dilettoso, C Anelli, D Balestri, C Graglia, Italy \\ Email: gianluca.dilettoso@tin.it}

Background: Cardiac surgery is advancing rapidly with benefits in terms of less invasive procedures; techniques are becoming more sophisticated to encourage a better functional recovery and improved cosmetic outcomes. Aim: Surgery to the mitral valve has traditionally been performed through median sternotomy. However, thanks to significant progress in video-assisted surgery, instruments and perfusion technology, mitral valve surgical procedures are now performed through gradually smaller and less invasive incisions, e.g. via left antero-lateral minithoracotomy using the heart portaccess technique. Nursing knowledge has encouraged the analysis of some critical issues that may arise during critical care nursing practice. Results: Patients who undergo cardiac surgery require "advanced monitoring" in the operating theatre directly, but also in critical and intensive care units, where sometimes this technique is widely employed. Nursing clincal practice and current literature have defined healthcare key issues to therefore adjust how nursing procedures are carried out. Monitoring techniques have been developed to identify and prevent at the proper time the onset of complications which may occur during the clinical pathway. These observations are both related to the traditional surgical approach and to the modern minimally invasive one. The key issues concerning patients who undergo cardiac surgery using the heart port technique are the monitoring of respiratory variables and the need of patients to breathe naturally. Implication for practice: During a minimally invasive cardiac surgery procedure using the heart port-access technique, nurses have to pay attention to the respiratory function of the patient in order to reduce potential respiratory complications. REFERENCES - Daniel J, Dattolo J, Minimally invasive cardiac surgery: surgical techniques and nursing considerations. Crit Care Nurs Q. 1998 Feb; 20(4): 29-39 • Modi P, Hassan A, Chitwood W.R., Minimally invasive mitral valve surgery: a systemic review and metaanalysis. Eur J Cardiothorac Sur. Nov 2008; 34(5):943:952.

Crisis Resource Management (CRM) training by simulation to improve safety in an intensive care unit

\section{Elin Drangsholt, Gyrid Aulie, Norway \\ Email: eldr@uus.no}

Background: Crisis Resource Management (CRM) teaches principles of managing critical situations and preventing errors in patient care. Key principles are leadership, effective communication skills and teamwork. Postoperative unit at Oslo University Hospital is an intensive care unit with postanaestethic, intensive care and trauma patients. The nursing staff consists of 60 Registered or Critical Care Nurses. The physicians are anesthetists on call. Simulation based education has been used regularly to train spesific skills like advanced cardiopulmonal resuscitation and endotracheal intubation. Effective teamwork outside these settings has not been trained systematically. In september 2010 all nurses were trained in CRM principles. The training consisted of theoretical and practical parts including simulation with case-scenarios and video-assisted debriefing. Aim: To improve teamwork, coordination and communication skills. Learning objectives of the course were defined according to the key points of Gaba's CRM concept. Results: Preliminary results from survey indicates improved skills of leadership, communication and teamwork. Implications for practice: The skills need to be regularly trained by all staff. The simulations motivated them to develop and practice the skills in their daily work. References: 1. Rall M, Dieckmann P: Crisis Resource Management to improve patient safety. European Society of Anaestehesiology, Abstract Euroanesthesia 2005. 2. Sundar E, Sundar S, Pawlowski J, et al: Crew Resource Management and team training. Anesthesiol Clin. 2007 Jun;25(2):283-300. 3. Issenberg SB, McGaghie WC, Petrusa ER, et al: Features and use of high-fidelity medical simulationthat lead to effective learning: a BEME systematic review. Med Tech. 2005 Jan;27(1):10-28.

Competence of graduating nurse students to practice in intensive and critical care nursing - what do the studies tell us?

Riitta-Liisa Lakanmaa, Tarja Suominen, Helena Leino-Kilpi, Finland

Email: riiaar@utu.fi

Background: There is globally an on-going need of new intensive and critical care nurses. Nursing education should respond to this demand, ensure an adequate supply of appropriately trained staff (cf. Robnett 2006) and collaborate with clinical practice in this challenge. Aim: Aim was to describe what kind of research there is of nurse students ' competence in intensive and critical care nursing. Methods: Literature review was done based on systematic search retrieved from national (Finland) Medic and international Medline, Cinahl and Eric databases. The literature was limited to English, years $2000-$ 2010 and abstract available. After analysis of titles and abstracts, $23(=n)$ research papers were included in the review. Results: The existing literature examines competence of nurse students from different perspectives and can be divided into six areas. There are research of 1) Nurse education programs (3) 2) Clinical practicum (4), 3) Teaching/learning methods (6) 4) Curricula evaluation (1) 5) Orientation/Internship programs (4) and 6) Competence/professional self-concept (5). Conclusion: There is lack of empirical research of graduating nurse students ' competence or competence assessment in intensive and critical care nursing. We need more evaluation research in nursing education of special fields such as intensive and critical care and also validated instruments for the evaluation of nursing education. References: Robnett, M.K. (2006). Critical care nursing: Workforce issues and potential solutions. Critical Care Medicine 34 (3), S25-S31.

The clinical simulation as an evaluation tool in postgraduate training of nursing in critical care and emergency

Concepció Fuentes-Pumarola, Josep Olivet Pujol, Pere Rimbau Muñoz, M. Àngels Galvany Ferrer, David Ballester Ferrando, Spain

Email: concepcio.fuentes@udg.edu

Aim: Determining the validity and reliability of clinical simulation as an evaluation tool of competences through an experience with nurses enrolled in postgraduate studies in critical care and emergences at the University of Girona. Methodology: Descriptive study of case series with the simulator: SimMan ${ }^{\circledR}$ where learning objectives, competences (correct treatment, nursing diagnosis) and the briefing are evaluated. Population: 180 Spanish postgraduate students with an average of 2-year experience in Emergences and Critical Care. Data collection period: 2008-2010. Results: A total of five cases have been considered, evaluating 7 learning objectives and 15 skills in each of them. The study includes the briefing (with audio and video recording) with the students. The average score was 7 out of 10. Conclusions: Clinical simulation through $\operatorname{SinMan}{ }^{\circledR}$ for competency assessment of nursing graduates with experience in emergences and critical care is a good tool for assessing the professional and for searching the potential areas for improvement. Keywords: Clinical competence, Simulation, Nursing, Education, Emergences, Critical Care. 
An adaptation of the Liverpool Care Pathway for the critical care setting

\section{Jodi Carpenter, Lindsay Franklin, United Kingdom \\ Email: jopo2000@hotmail.com}

An adaptation of the Liverpool Care Pathway for the Critical Care Setting In the United Kingdom, the Liverpool Care Pathway (LCP) is the gold standard for end of life $(E O L)$ care. It is a "multi-professional tool that has been designed to improve and standardise care for dying patients in all health care settings" (Preston 2007 Pg 61). As critical care is a specialist setting, the LCP was adapted as a care bundle to meet the needs of dying patients (Berenholz et al 2002). Reasons for adapting the LCP included: 1 . Computerised documentation 2. Provision of checklist to a. Address active withdrawal of treatment (WDT) b. Ensure high quality EOL care c. Quick reference when EOL comes rapidly 3 . Audit tool "A (Care) bundle is a specific tool with clear parameters. It has a small number of elements that are all scientifically robust, that when taken together create much improved outcomes." (Institute for Healthcare Improvement 2006). Our EOL care bundle was introduced in 2007 to guide nurses to meet EOL goals, as set out in the LCP. The Care Bundle is audited monthly ensuring best practice, demonstrating lack of knowledge in specific areas which are addressed in an ongoing educational programme. The introduction of the EOL care bundle, with associated protocols and teaching, ensures high quality care for the dying patient, effective support for relatives and empowers staff to be confident in their practice. References Berenholtz et al (2002) Quality review of intensive care unit quality indicators. Journal of Critical Care 17(1): 1-12. Institute for Healthcare Improvement (2006) What is a care bundle? [Online] IHI, 2006. Available at: www.ihi.org/lHI/Topics/ CriticalCare/IntensiveCare/ImprovementStories/WhatlsaBundle. $\mathrm{htm}$ (Accessed 01/10/10) Preston, M (2007) The LCP for the dying patient: a guide to implementation. End of Life Care 1(1): 61-68.

Nursing students employed to watch over critical ill patients in need of continuous surveillance. Reducing costs and improving nursing recruitment in ICU

\section{Susanne Hermansen, Gitte Mlkkelsen, Eva Lærkner, Denmark}

Email: susanne.lykke.hermansen@ouh.regionsyddanmark.dk

Background For several years our ICU has used trained medical students to perform duties as nurses assistants with critical ill patients in need of continuous surveillance (e.g. non-sedated intubated children, agitated patients). Recently an initiative has been introduced, in which nursing students are offered a similar employment after undergoing a training course. This change was made partly to reduce cost and partly for recruitment purposes. Aim To examine whether the nursing students were perceived qualified to perform the tasks required after their training. To document financial advantage from using nursing students compared to medical students. To explore if the ICU job experience affected the students' choice of employment after graduation. Methods A questionnaire was given to all students, who had worked at least 5 shifts on ICU, and all nurses employed at the time of the project. Costs were monitored for 6 months and compared to similar expenses using medical students. Nursing students, who graduated, were given a questionnaire exploring their future intention about working in an ICU. Results The nursing students were perceived to be qualified to some extend to perform the tasks as nurses assistants after the training course, but they needed more practical experience. The cost analysis using nursing students instead of medical students showed a cost reduction of more than $30 \%$ according to each 8 hour shift. All graduated students, who have responded express an interest in working on ICU. 2 students have been employed in the department directly after graduating. Implications for practice It is possible to employ Nursing students instead of Medical students on ICU-wards as nurses assistants to watch over critical ill patients in need of continuous surveillance. Employing nursing students reduced the cost and may increase the knowledge about critical care among Nursing students and thus improve nursing recruitment in ICU.

Dependance of peak/PEEP pressures and intra-abdominal pressure/gastric residual volume in mechanically ventilated and enterally fed ICU patients

\section{Liilia Leius, Svetlana Frolova, Boris Gabovits, Estonia \\ Email: liilia.leius@itk.ee}

Question of study: Is intraabdominal pressure, food absorbtion in gastrointestinal tract and amount of gastric residual volume in intensive care patients who are simultaneously on mechanical ventilation in dependence with PEAK and PEEP pressure? Aim of study: Aim of the study was to clarify intensivecare patients with enteral nutrition who were on mechanical ventilation throughout the whole periood of study and in whom intraabdominal pressure and gastric residual volume was measured. Selection: Study has been performed in East Tallinn Central Hospital in intensive care patients from 01.02.2010 till 01.09.2010. 248 intensivecare patients participated in study, from which 122 intensivecare patients were on spontaneous ventilation and 136 intensivecare patients were on mechanical ventilation. Spedasine study focused on mechanically ventilated ICU patients, in which 109 were enterally nourished and 25 weres not fed/nourished enterally. Method: Patients data sheet has been used to collect data. Data was processed by Microsoft Excel and PASW programs. Results: Procressing the data revealed that in ICU patients intraabdominal pressure, food absorbtion and amount of gastric residual volume are not depended on PEAK and PEEP pressures of mechanical ventilation.

Study of the efficacy of an enteral nutrition algorithm in critical adult critically ill patients

Concepció Fuentes-Pumarola, Maria Colomer Plana, Carles Cordon Bueno, Guadalupe García Cuesta, Margarita Montiel Váquez, Sónia Puig Codina, Montse Sala Martí, Spain

Email: concepcio.fuentes@udg.edu

Aim: Evaluation of the effectiveness of an enteral nutrition algorithm in adult critically ill patients, by monitoring prealbumin, albumin, total protein and energy contribution. Methodology: Design: prospective observational study. Population: 30 patients admitted to a Spanish intensive care unit for adults who receive NE. Data collection period: 10 February to 14 June 2010. Results: The three biochemical parameters monitored at admission or the first week with $\mathrm{NE}$, has an average lower than the range considered normal. At the end of the protocol or the other of these parameters improve patients but only achieves normal prealbumin. The increase is significant in the case of prealbumin and total protein $(p=0.014<a$ ). The protocol / algorithm achieves an average contribution of $24.72 \mathrm{kcal} / \mathrm{kg}$ / day. Conclusions: The effectiveness is demonstrated to obtain a significant increase in total protein and prealbumin values since the beginning of the NE or to the high end of the protocol / algorithm. While the calorie content is the 3rd day they recommend increasing scientific evidence $\mathrm{kcal} / \mathrm{kg} /$ day from the third day is statistically significant. Keywords: Enteral nutrition, critical illness, intensive care units, calorie intake, prealbumin.

Where your patient comes from, is it important?

\section{Sabrina Egman, Italy}

Email: segman@ismett.edu

Background of Problem: Our Institute mission and a particular geographic collocation, want that, the $85 \%$ of our patient don't come to our country. Not only the nationality is different; they have a 


\section{*uropean Critical Care Nursing: Working Together for a Better Tomorrow.}

2011 congress abstracts *

different cultur, social-cultural background,a different life approach, and a different to live a pathology situation, and of course a different language. The families, have a big logistical discomforts, with a long time far from home, the family goal is the best future life and the most important think: the health. Goals of Project Action:. In order to guaranty at our foreign patients the high level of care, for nursing quality and support; we build a professional collaboration net to have a strong help communication near at Hospital with the international community, in our city, but also at home. We estabilish a contact with a hospital in Country where the patients come from, then when they will come back home, the hospital can take care to them for the follow up. The good relationship is the most important think in oder to fix all the history and the problems of the patients Interventions: There is a dedicate an International patient coordinator, he is a point of reference for all the team of care, in order to have a strong support in communication with family and patients we need: Interpreters; - Cultural intermediator; - Social worker; - Psycologist; Conclusions: In order to take care to International patient, with a high level of standard, we under stand that we need the support from other health provider. We build a bridge for International patient in our country. Learning Objectives: The participant will be able to: 1. Discuss the potential solution of communication problems with international patients 2. Discuss how to implement a support strategy in international population.

\section{Families becoming integral members}

\section{Sabrina Egman, Italy}

Email: segman@ismett.edu

Studies have found that family presence at the bedside of critically ill and injured patients during invasive procedures and resuscitation can positively affect patient outcomes and family satisfaction. Despite this evidence, many critical care units do not permit family presence at these crucial times and limit visitation throughout the patient's ICU stay. Purpose: To equip the participant with tools to integrate families as requisite members of the healthcare team in both adult and pediatric transplant patients in the critical care units. Strategies to achieve this goal include case studies and scenario exploration with small group audience participation. Key components of this session include the concepts of family-centered care and how to create and utilize evidence-based family presence policies. The pros and cons of creating and supporting family presence from a nursing perspective will be explored. Finally, small groups will examine scripted resuscitation/invasive procedure scenarios from the perspective of the nurse, the healthcare team, the family, and the patient. The groups will explore their feelings and insights gained through the exercise, and will share the findings with the entire group. The target audience is the acute and critical care nurse that desires to be a catalyst for the integration of family-centered care and desires to include families as integral members of the team. No prerequisite knowledge is necessary. Outcomes for the participant include an enhanced understanding of family-centered care, including the unique perspectives of others attending the session. Participants will leave the session with diverse strategies to take back to their workplace.

The nursing documentation in ICU: which indicators to verify the quality

\section{Sabrina Egman, Italy}

Email: segman@ismett.edu

Introduction: The basic structure of the electronic health record is used to prepare and review the medical records, consisting mainly, as the requirements from clinical, pharmacological and laboratory, the assessments of clinical staff. Within a defined business strategy and the relative path to achieving accreditation of our institute according to standards the Joint Commission International, the Nursing documentation plays a crucial role. Objectives: - Improve quality of nursing documentation - Reduce clinical risk; - Improve the efficiency of the system and the support for research; Increased accessibility and transparent. Materials and Methods: The implementation was established by a clinical project, which was supported by the Department of Information Technology for the technological infrastructure, which was followed by the actual configuration that ended with the staff training. The specific model of nursing assessment, aims to provide a valuable tool for the documentation, but also for the development of a multidisciplinary treatment plan customized. The main areas iare: - Neurological - Cardiovascular - Respiratory - Genitourinary - Gastrointestinal Integumentario - Psychosocial and educational For each area were made of micro-air, with the ability to insert your own comments, but with a grid of possible assessments already present, representing the desired quality standards. Results: The advantages of using an EHR, were the improvement of accessibility, availability and quality of information, as well as his uniform, compared with a reduction of time spent on data collection, reduction in human error. Conclusions: The nursing assessment if done standards-based gain a better interpretation, giving consistent care attitude, enriching, especially when the path that led to the development of this tool stems from the clinical component, as it will not only desire implement innovative technologies, but will expand exponentially enriching the value of the documentation with the characteristic of our critical thinking skills.

Urgent words of caring: a reflective writing workshop in an Italian emergency medicine ward

\section{Massimo Michele Greco, Marco Martelloni, Chiara Carruba,} Maddalena Galizio, Italy

Email: massimo.greco@ptvonline.it

Health Personnel professional self-fulfillment is beyond the performance of procedural technical skills, but is related also with the ability to "take care" of the stories of caring, to find a more complete meaning of it and to highlight their "tacit knowledge" and their relational competences. In order to verify if a reflective writing group could be a mean to create a space for emotional and cognitive learning for the nursing staff of a post-Emergency ward, a writing workshop was designed and monitored in its ongoing process. To develop it, the Narrative Medicine theoretical framework was chosen and adapted to nursing paradigm. An expert in Autobiographical and Narrative Medicine methodologies designed a 8 steps workshop (from July to October 2010), directed to 9 nurses of the Department of Emergency Medicine, University Hospital of Tor Vergata, Rome Italy. The sessions, two hours each, had a focus topic that varied from the "individual self", "professional self", "the relationship with the patients", "the relationship within the team". The purpose was also to define a model of reflective writing permanent activity, to be implemented by the staff as a part of the professional routine. The autobiographical texts written by participants, and read in group sessions, brought out professional considerations, existential perspectives, emotional and relational experiences. At the end of the course, participants were able to decide which kind of narrative tools could be interesting to reach more fully professional goals. References Charon, R. (2006). Narrative Medicine - Honoring the Stories of Illness. New York: Oxford University Press . Greco, M. M. (2008, Jan-Feb). Prendersi cura delle storie di malattia. Pedagogika. it (1), pp. 17-19. Jones, C., \& Freshwater, D. (Eds.). (2005). Transforming Nursing through Reflective Practice (2nd ed.). Oxford, UK: Blackwell Publishing. 


\section{Exchange program}

\section{Eva Barkestad, Sweden}

Email: evabarkestad@bredband.net

EfCCNa exchange program The aim in the EfCCna exchange program is to enable critical care nurses to visit critical care units in other European countries and spend some time on a particular unit of their choice. All critical care nurses, or nurses working in critical care units, from European countries who are members of EfCCNa can participate in the program. Critical care nursing in Europe varies from country to country. Objectives are to stimulate professional development and sharing expertise in collaboration of critical care nurses. It is also to increase the participants' understanding and tolerance of other cultures, and broadening their social horizons. Since 2006 a total of thirty-seven nurses from different countries have taken part in the exchange program. This presentation will tell you more about the program and the evaluation of their stay from the nurses who participated in the program.

Intensive care units "without walls" - concept "Outreach"

\section{Gordana Dragosev, Sofija Alijevic, Petar Mirkovic, Serbia}

Email: dragosev@gmail.com

INTRODUCTION: The advances in technology and demands for intensive care beds, means that many critically ill patients are increasingly being treated general wards. However, Intensive Care Nurses have well developed knowledge, expertise and clinical competencies in caring for critically ill patients. This depth of knowledge and skills is maintained through on-going educational activities Although the mortality of critically ill patients significantly reduced, many patients experiencing cardiac arrest tend to occur outside the intensive care environments. This situation might be avoided, if ward nurses were able to rapidly and accurately recognize patients most at risk for cardiac arrest. AIM: Promote the concept of Intensive Care Unit "Without Walls", which aims are to transfer knowledge's and skills from ICU Nurses to departments Nurses. METHODS: Data from the literature, interviews with ICU and departments Nurses of Serbia, the questionnaire CONCLUSION: Creating and accepting the concept of intensive care "without walls", ICU Nurses become educators, consultants and, as members of the team for rapid resolution of problematic situations, they are directly involved in the treatment of critically ill / injured patients at the departments. Knowledge and skills that apply in the ICU become available to everyone, not just in the premises of the ICU. KEY WORDS: concept "Outreach", Critical Care, Nursing REFERENCES: Clinical Effectiveness of a Critical Care Nursing Outreach Service in Facilitating Discharge from the Intensive Care Unit - American Journal of Critical Care. 2010;19(5):e63-72. ( 2010 American Association of Critical-Care Nurses Intensive Care "Without walls " Dr Ivan Palibrk, National Congress UINARS, Serbia 2008., Abstract book

Nurses' resistance to changes in health informatics and technology

\section{Gordana Dragosev, Sanja Paunic, Serbia}

Email: dragosev@gmail.com

INTRODUCTION: In the new dynamic environment of innovative companies, all organizations are expected rapid adaptation to change and move to a higher level. As most health workers are Nurses, their role in the implementation of the informational - technological change is of great importance. It is difficult to change the support pillars, and not destroy the whole system. Whether Nurses work in intensive care, operating room or in primary Health Care system, they are constantly exposed to new and more complex tasks with ever shorter period of realization. If we add a new manner of data recording, evaluation, quality monitoring, e-learning and if we have not prepared the ground for change, one thing is certain: we'ill find resistance, defensiveness, fear, conflict. AIM: To point out the importance of a systematic approach to introducing changes, teamwork and move Nurses inert system into a dynamic environment that can easily accept changes. METHODS: Descriptive and displaying methods for changing management which we suggest, named „8 stages change process“ and „5 steps to overcome resistance". CONCLUSION: In health care today, resisting changes and ignoring the unstoppable expansion of new diagnostic and therapeutic procedures, can have serious consequences both for the health institutions and health workers, and for recipients of health care (inadequate use and handling of medical devices and computer technology or inadequately observed therapy in a computer system, can be fatal for the patient). An honest attitude, knowledge, communication and involvement of all employes are the key factors in managing Nurses resistance to changes in Health Informatics and Technology. Keywords: resistance, change management, health informatics References: TIMMONS S. Nurses resisting information technology, Nursing Inquiry 2003; John P. Kotter Leading Change, Harvard Business School Press, 1996.

\section{Stress among Serbian critical care nurses}

\section{Gordana Dragosev, Dragana Milutinovic, Nina Brkic, Serbia}

Email: dragosev@gmail.com

Introduction: Nursing is acknowledged to be a stressful profession worldwide, and Serbia is also acknowledged as a highly stressed society. Aim: Present research were analyzed causes of work related stress on critical care nursing staff in Serbian health institutions as well as evaluating their well being and designing plan of its prevention. Methods The research was conducted as a cross-sectional study. The sample included $(n=1000)$ nurses. Expanded Nursing Stress Scale and an additional questionnaire created for the purpose of this investigation were used as research instruments. Results Cronbach alpha coefficient for the whole scale was 0.94 . The average interitem correlations were 0.22 , and item-total correlations ranged from 0.20 to 0.60 . Descriptive analysis of 57 potentially stressful situations at work in the intensive care unit nurses, the most stressful work experience in which they are exposed to health hazards and safety $M=3.17 \pm 0.91$ (SD), and the death of patients with whom they have developed a close relationship $M=3.13 \pm 1.02$ (SD). ANOVA analyses were statistically significant differences in the perception of some stressful situations in relation to age, marital status and education.. Conclusion The conclusion is that stress is present in nurses' activities in intensive care causing dissatisfaction and stressrelated symptoms. It is important to promote condition of work (work space, providing new equipment), organization of work, as well as social relate, in accordance with the recommendations of ICN. References: 1. Cavalheiro AM, Moura Junior DF, Lopes AC. Stress in nurses working in intensive care units. Rev Latino-am Enfermagem 2008; 16(1):29-35 2. French S. E, Lenton R, Walters V, Eyles J. An empirical evaluation of on Expanded nursing stress scale. Journal of Nursing Measurement 2000; 8(2): 161-178 Key words: Stress, Workplace; Nurses, Questionnaires.

Fellowship for specialist nurses in anesthesia and intensive care

\section{Eva Âkerman, Roland Nilsson, Lotti Orvelius, Ingrid Andersson,} Sweden

Email: akerman@home.se

Fellowship for specialist nurses in anesthesia and intensive care Background An increased population of elderly and demands for more complex treatments, means an increased degree of specialization and subspecialization in health care - not least in the areas of anesthesia and critical care. The increasing complexity of care and medical equipment emphasises the need for knowledge 
and understanding of the physiological processes. This will demand for new requirements of specialist nurse education. Aim to create an postgraduate education for specialist nurses, with a focus on clinical activity, with increased quality and safety in bedside patient care. Results National association of Anesthesiology and Intensive Care has developed a Fellowship-program consisting of five sections; clinical work, a practice-oriented clinical specialization, planned, varied and regularly reflected through mentoring - development work, in which participants will acquire advanced knowledge in a systematic process work - exchange programs, in which participants will reflect their own performance in a similar activity with a different cultural perspective - theoretical depth, in which participants acquires skills that enables them to function as a resource person - conscious individual leadership, knowledge of why, the contexts in which, how, what qualities I have and how could I lead Implications of practice The Fellowship-program enhance advanced training and should be accompanied by the opportunity to make a clinical career. The creation of positions with the title clinical nurse specialist, or clinical expert nurse may be an action in this direction, which in the workplace will recognize the participant extended competence. The post makes it clear that the skills are desired, important and to be used in clinical practice. The position is expected to serve as an important resource and a role model for nurse colleagues.
Giving voice to loved ones: using Photo-Voice as a data collection method

\section{Sepideh Olausson, Berit Lindahl, Margaretha Ekebergh, Sweden}

Email: sepideh.olausson@hb.se

The Intensive Care Unit (ICU) is a place of care for the most critically ill patients. The ICU rooms are considered to be one of the most complicated rooms to design. Providing successful intensive care requires that human, technologies and spatial resources are integrated in a rigorous way. Research shows that being a patient or next-of-kin in ICU is a traumatic experience not only because of the illness but also because of one's human existence is threatened. Literature suggests that the presence of next-of-kin and the design of ICU affect the patients' recovery and wellbeing. Aim: The aim of this study as a part of a larger research project was to illuminate nextof-kin's experiences of the physical environment in patient's room in ICU. Method: Data were collected through qualitative research interviews in combination with a photo-voice method(Colleen et al., 2008). The next-of-kin were invited to take photos of various aspects of the room that they associated with a feeling. Data were analyzed using a phenomenological hermeneutics method. Result and conclusion: The room is seen as a safe and an emotional place to dwell in. People, relations and the interiors build up the atmosphere in the room. Being in this room means to try to create a temporary living-room different to ordinary life and try to inhabit the room both as a physical place and a lived space. It also means being in a state of uncertainty and homelessness. Some of the photos will also be presented as well as a short discussion about the data collection method. Colleen, K., Fleury, J.,Perez. A., Ainswoth. B. \& Vaughan. L (2008). Using visual methods to uncover context. Qualitative Health Research, 18(3), 428-436. 